This dissertation has been 65-38:32 microfilmed exactly as received

CHANHY, Robin Ward, 1938-

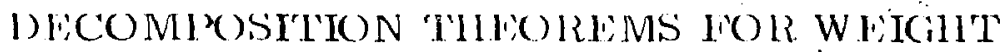
IPUNC'TIONS IN TIRANSTORMATION 'THHORY IV)R MUASURR SIIACL.

The Ohio State University, lh.l)., 1964 Mathomatics

University Microfilms, Inc., Ann Arbor, Michigan 


\section{DECOMPOSITION THEOREMS : FOR WEIGHT \\ FUNCTIONS IN TRANSFORMATION THEORY \\ FOR MEASURE SPACE}

\section{DISSERTATION}

\section{Presented in Partial Fulfillment of the Requirements for the Degree Doctor of Philosophy in the Graduate School of The Ohio State University}

By

Robin Ward Chaney, B. Sc.

The Ohio State University. 1964

Approved by

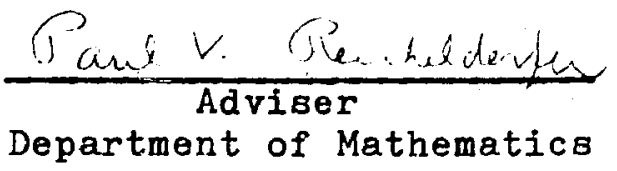


VITA

December 13, 1938 Born - Cleveland, Ohio

1960

B.S., Ohio State University, Columbus, Ohio

$1960-1962$

National Science Foundation Fellow,

Department of Mathematics, The Ohio State

University, Columbus, Ohio

$1962-1964$

Graduate Assistant, Department of Mathematics,

The Ohio State UnIVersity, Columbus, Ohio

FIELDS OF STUDY

Major Field: Mathematics

Studies in Analysis. Professors P. V. Reichelderfer and

R. G. Helsel

Studies in Algebra. Professors H. J. Ryser and Erwin Kleinfeld

Studies in Topology. Professors P. V. Reichelderfer and Norman Levine 


\section{CONIENTS}

Page

INTRODUCTION

Chapter

I. PRELIMINARIES

II. THE MAIN DECOMPOSITION THEOREMS

III. ON WEIGHT FUNCTIONS SATISFYING H13

SUMMARY

BIBLIOGRAPHY
6

112

142

1

148 


\section{INTRODUCTION}

In 1961 Reichelderfer published a paper entitled "A Transformation Theory for Measure Space." The present work consists of contributions to this new abstract transformation theory. In turn Reichelderfer's paper has as its antecedent the book entitled Continuous Transformations in Analysis. This book was written jointly by Rado and Reichelderfer and was published in 1955. One of the main purposes of that book was to obtain powerful results concerning the transformation of integrals; the results obtained both include and extend the known classical transformation formulas.

The results in [2] (the number in [ ] refers to the bibliography) are developed in a setting which we shall now describe briefly; a thorough description of this setting is found in Chapter $I$ of this paper. Let $T$ be a function defined on a non-empty set $\underline{S}$ and suppose that $\underline{S}^{\prime}=$ TS. Let $\{\underline{S}, \underline{M}, \mu\}$ and $\left\{\underline{S}^{\prime}, \underline{M}^{\prime}, \mu^{\prime}\right\}$ be measure spaces. Suppose that $\underline{D}$ is a collection of subsets $D$ of $\underline{S}$ and that $W^{\prime}$ is a non-negative function defined on $\underline{S}^{\prime} \times \underline{D}$. Assume that $T,\{\underline{S}, \underline{M}, \mu\},\left\{\underline{S}^{\prime}, \underline{M}^{\prime}, \mu^{\prime}\right\}, \underline{D}$, and $W^{\prime}$ satisfy certain standard conditions; under these conditions $W$ ' is termed a weight function for $T$. Within this basic setting Reichelderfer presents in [2] necessary and sufficient conditions in order that there exist a non-negative (extended real 
valued) function $f$ defined on $S$ and $\mu$-integrable such that

$$
\int_{D} f(s) d \mu=\int_{S^{\prime}} w^{\prime}\left(s^{\prime}, D\right) d \mu^{\prime}, \quad D \in \underline{D} .
$$

Whenever these conditions are satisfied it is shown that a transformation formula holds in the following sense:

Let $H^{\prime}$ be a real valued $M^{\prime}$-measurable function defined on $\underline{S}^{\prime}$. Then for each $D$ in $D$ the function $H^{\prime}\left(s^{\prime}\right) W^{\prime}\left(s^{\prime}, D\right), s^{\prime} \in S^{\prime}$, is $\underline{M}^{\prime}-$ measurable and the function $\mathrm{H}^{\prime} \mathrm{T}(s) f(s), s \in \underline{s}$, is M-measurable. The former function is $\mu^{\prime}$-integrable over $\underline{S}^{\prime}$ if and only if the latter function is $\mu$-integrable over $D$. When these functions are integrable the transformation formula

$$
\int_{D} H^{\prime} T(s) f(s) d \mu=\int_{S^{\prime}} H^{\prime}\left(s^{\prime}\right) W^{\prime}\left(s^{\prime}, D\right) d \mu^{\prime}
$$

holds. The paper also examines an important special class of weight functions. It ends with an outline of the relationship between the results of [2] and [I] together with an application to Euclidean space. Indeed it is shown that the general transformation formula in [1] is a special case of (1).

The work in [2] constitutes not only an extensive generalization of large parts of [1] but also an unexpected simplification. Rado and Reichelderfer developed in [I] a transformation theory for continuous transformations defined on bounded domains in Euclidean n-space and having bounded ranges in Euclidean n-space. Additional conditions are imposed upon the weight functions W'. Moreover the topological properties of Euclidean n-space are used abundantly in [1]. Craft was able to remove these additional conditions on W'; and then, in [2], Reichel- 
derfer was able to eliminate all overt use of topology. Some of the standard hypotheses in. [2] have obvious topological forerunners. Nevertheless no topologies appear in the discussion. Thus the results in [2] remove the transformation formulas from the realm of Euclidean space and even from the much broader realm of topology. The consequences of this generalization have not yet been studied extensively. Furthermore there is more flexibility in Euclidean applications. For example, the results of [1] cannot be applied in the case of a continuous transformation from n-space to $n^{\prime}$-space with $n \neq n^{\prime} ;$ however, the results in [2] can be applied in this case.

The present work has [2] as its foundation. Throughout almost all of this dissertation we shall suppose that we are working with measure spaces $\{\underline{S}, \underline{M}, \mu\}$ and $\left\{\underline{S}^{\prime}, \underline{M}^{\prime}, \mu^{\prime}\right\}$, a transformation $T$ from $\underline{S}$ onto $\underline{S}^{\prime}$, and families $\underline{D}$ and $\underline{B}^{\prime}$ which satisfy the standard hypotheses HI - H8 set forth in [2]. Our interest will be centered chiefly upon weight functions (for T). The main objective is to obtain decompositions of weight functions as sums of certain other weight functions each having special properties. These decompositions have as their model the classical Lebesgue Decomposition Theorem for measures (see [3]). In the course of establishing these results we shall prove some properties of weight functions which are themselves of independent value. There is also a result which falls into the realm of abstract transformation theory but which seems quite unrelated to the main objective. Thus the paper has a dual purpose. We 
have not only the aim of obtaining certain decomposition theorems but also the broader goal of making general (sometimes scattered) contributions to the new transformation theory ([2]).

With these general remarks set forth we can now briefly describe the progression of the paper. In Chapter I certain preliminary concepts and lemmas are presented. Several broad conventions àre established at the outset of this preliminary chapter. Then the standard hypotheses of [2] are listed along with some new standard hypotheses. The basic definitions, lemmas, and theorems of [2] are also listed for the reader's convenience. The chapter concludes with the presentation of some definitions and preliminary lemmas which appear here for the first time.

The first part of Chapter II is devoted to the proof of the General Decomposition Theorem for weight functions. This result has the virtue of being valid under very general conditions. It has a limitation also; one of the two "components" of the decomposition is not at all well classified. The next portion of Chapter II is devoted to the elimination of this drawback. The result is the Strong Decomposition Theorem. This last theorem requires however that a rather broad additional condition be imposed upon the weight function W'. The chapter concludes with the discussion of some germane examples.

Chapter III deals with the important special class of weight functions which satisfy $\mathrm{Hl} 3$ of [2]. This class was introduced first in [2] and it includes all of the weight functions 
(or, admissible multiplicity functions) considered in [l]. In the first part of the chapter we prove several "measurability" results" of far-reaching significance. Then we apply the results of Chapter II to obtain the Special Decomposition Theorem. We observe that the resulting decomposition assumes a particularly desirable form in this case. The chapter then concludes with a section that is unrelated to the main theme of the dissertation. The value of the results of this last section remains unknown.

Finally we present a concise Summary.

Some of the results of this paper are due entirely to Professor Reichelderfer, the writer's adviser. This fact is noted whenever such results appear. 


\section{CHAPTER I}

\section{PRELIMINARIES}

In Chapter I the basic mathematical, system with which this dissertation is concerned is set forth in detail. Section I.I establishes some basic notational conventions. Section I.2 includes the basic hypotheses and definitions of transformation theory which are introduced in [2]. Theorems and lemmas from [2] which are used explicitly in this dissertation are listed in Section I.3. Finally, Sections I.4 through I.7 consist of certain preliminary conventions, definitions, and lemmas which are essential to this paper and which appear here for the first time.

\section{I.I General conventions}

I.1.I. Remarks. Throughout this paper $\mathrm{R}^{\mathrm{n}}$ denotes Euclidean n-space. $R_{+}$always denotes the set $\left(t \in R^{I}: t \geqq 0\right) U$ $U\{\infty\}$. The symbols $\infty$ and $+\infty$ are used interchangeably. The symbol $-\infty$ is never used. We adopt the following conventions concerning $\infty$ :

$$
\begin{aligned}
& \text { i) } \infty+a=\infty \text { if a is in } \mathrm{R}^{1} \text {. } \\
& \text { ii) } \infty+\infty=\infty . \\
& \text { iii) } \infty-a=\infty \text { if a is in } \mathrm{R}^{1} \text {. }
\end{aligned}
$$




$$
\begin{aligned}
& \text { iv) } \infty \cdot 0=0 . \\
& \text { v) } \infty \cdot a=\infty \text { if a is in } R_{+} \text {and if } a>0 . \\
& \text { vi) } a<\infty \text { if a is in } R^{1} .
\end{aligned}
$$

Other conventions are common but we shall not need them.

I.1.2. Remarks. Let $M$ be a non-empty subset of $R^{I} \cup R_{+}$.

The least upper bound of $M$ or supremum of $M$ is denoted by sup $M$. The greatest lower bound of $M$ or infimum of $M$ is denoted by inf $M$. I.1.3. Remarks. We shall frequently be concerned with functions which assume values in $R^{l}$ or in $R_{+}$. A function whose values lie in $R^{I}$ is termed a real valued function. A function whose values lie in $R_{+}$is termed a non-negative function.

I.1.4. Remarks. In this dissertation we shall often work with sets denoted by $\underline{S}$ and $\underline{S}$ '. If $E \subseteq \underline{S}$ we define $C E$ to be the set of objects in $\underline{S}$ but not in $E$. If $E^{\prime} \subseteq \underline{S}^{\prime}$ we define $C^{\prime} E^{\prime}$ to be the set of objects in $\underline{S}^{\prime}$ but not in $\mathrm{E}^{\prime}$. If $\mathrm{E} \subseteq \underline{S}$ then $\mathrm{H}_{\mathrm{E}}$ denotes the characteristic function of $\mathrm{E}$ as a subset of $S$; it is defined by

$$
\mathrm{H}_{\mathrm{E}}(s)\left\{\begin{array}{ll}
=I & \text { if } s \in \mathrm{E} \\
=0 & \text { if } s \in \mathrm{CE}
\end{array}, \quad s \in \underline{S}\right. \text {. }
$$

Similarly, if $\mathrm{E}^{\prime} \subseteq S^{\prime}$, then $\mathrm{H}_{\mathrm{H}}^{\prime}$, denotes the characteristic function of $E^{\prime}$ as a subset of $\underline{S}^{\prime}$. 
I.1.5. Remarks. Many concepts of measure theory are used in this paper. See reference [3] for definitions and theorems concerning the concepts of measure and integration which appear in this paper.

\section{I.2 Standard hypotheses}

In this section the basic hypotheses are introduced. They are listed as HI through H15, HI5i, H4*, H7*, and HCl through HC4. Some basic definitions from [2] are presented and several notational conventions are established.

I.2.1. We list the standard hypotheses of [2] along with several definitions.

Hl. $\{\underline{S}, \underline{M}, \mu\}$ is a $\sigma$-finite complete measure space.

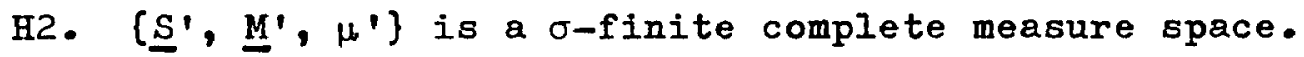
H3. T is a single valued transformation from $\underline{S}$ onto $\underline{S}$ ' H4. D is a collection of subsets $D$ of $\underline{S}$ with the following properties: The empty set $\varnothing$ and $S$ are in $D, D \subseteq \underline{M}$, and $\underline{D} \subseteq \underline{M}^{\prime}$ where $\underline{T D}=\{T D: D \in \underline{D}\}$. If ${ }_{1} D$ and $2^{D}$ are sets in $\underline{D}$ there exists a countable number of pairwise disjoint sets $D_{1}$ in $\underline{D}$ such that ${ }_{1} D \cap 2^{D}=U D_{i} \cdot \underline{S}$ can be expressed as the union of a countable number of sets ${ }_{*} D_{j}$ in $\underline{D}$ such that $\mu_{*} D_{j}$ is finite for every $j$, and such that if $M$ is in $M$ with $M \subseteq{ }^{D}{ }_{j}$ for some $j$ then for every $\varepsilon>0$ there exists a countable number of pairwise disjoint sets $D_{i}$ in $\underline{D}$ such that $M \subseteq U D_{i}$ and $\Sigma \mu D_{i}<\mu M+\varepsilon$. $H^{*}$. $\underline{D}$ is a collection of subsets $D$ of $\underline{S}$ with the 
following properties: The empty set $\varnothing$ and $\underline{S}$ are in $D, \underline{D} \subseteq \underline{M}$, and $\underline{T D} \subseteq \underline{M}^{\prime}$. If $I^{D}$ and ${ }_{2} D$ are sets in $\underline{D}$ there exists a countable number of pairwise disjoint sets $D_{i}$ in $D$ such that ${ }_{1} D \cap{ }_{2}^{D}=U D_{i}$. If $M$ is in $M$ and if $\zeta>0$ then there exists a countable number of pairwise disjoint sets $D_{i}$ in $\underline{D}$ such that $M \subseteq U D_{i}$ and

$\Sigma \mu D_{i} \leqq \mu M+\zeta$

Remark. It is noted in [2] that $\mathrm{H}^{4}$ * implies $\mathrm{H} 4$.

Definition. A set $D_{0}$ in $\underline{D}$ is said to be of type $Y$ if it is. one of a countable number of pairwise disjoint sets $D_{i}, i \geqq 0$, for which there exist two subsets $Y$ and $Z$ of $\underline{S}$ such that

$$
\begin{aligned}
& \text { i) } \underline{S}=Y \cup Z \cup U_{i \geqq 0} D_{i} ; \\
& \text { ii) } \Psi \in \underline{M}, \mu Y=0 ; \\
& \text { iii) } \mathrm{TZ} \in \underline{M}^{\prime}, \mu \cdot T Z=0 . \\
& \text { H5. If } D \text { is in } \underline{D} \text { there exists a sequence of sets } D_{j} \text { in }
\end{aligned}
$$

D such that

$$
\begin{aligned}
& \text { i) each } D_{j} \text { is of type } Y \text {; } \\
& \text { ii) } D_{j} \subseteq D_{j+I^{\prime}}, j \geqq I ; \\
& \text { iii) } D=U D_{j^{*}}
\end{aligned}
$$

H6. B' is a o-field of subsets B' of S' with the following properties: $\underline{B}^{\prime} \subseteq \underline{M}^{\prime}$ and $T^{-1} \underline{B}^{\prime} \subseteq \underline{M}$. For each set $M^{\prime}$ in $\underline{M}^{\prime}$ there exist sets $B_{1}^{\prime}$ and $B_{2}^{\prime}$ in $\underline{B}^{\prime}$ such that $B_{1}^{\prime} \subseteq M^{\prime} \subseteq B_{2}^{\prime}$ and $\mu^{\prime} B_{1}^{\prime}=\mu^{\prime} B_{2}^{\prime}$. 
Remark. It is observed in [2] that we may even require $\mu^{\prime}\left(B_{2}^{\prime} \cap C^{\prime} B_{I}^{\prime}\right)=0$ in the last condition of $\mathrm{H} 6$.

Definition. Denote by (D' all the subsets $O^{\prime}$ of $\underline{S}^{\prime}$ for each of which there exists a countable number of pairwise disjoint sets $D_{i}$ in $\underline{D}$ such that $T^{-1} O^{\prime}=U D_{i}$.

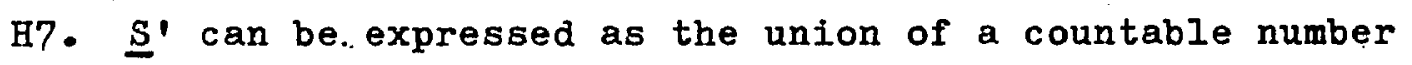
of sets ${ }_{*} O_{j}^{\prime}$ in $\mathbb{D}^{\prime}$ such that $\mu^{\prime}{ }_{*} 0_{j}^{\prime}$ is finite for every $j$ and such that if $M^{\prime}$ is in $M^{\prime}$ with $M^{\prime} \subseteq{ }^{*} O_{j}^{\prime}$ for some $j$ and if $\mu^{\prime} M^{\prime}=0$ then for every $\varepsilon>0$ there is a set $O^{\prime}$ in $\mathbb{D}^{\prime}$ such that $M^{\prime} \subseteq 0^{\prime}$ and $\mu^{\prime} O^{\prime}<\varepsilon$.

H7*. If $M^{\prime}$ is in $\underline{M}^{\prime}$ and if $\zeta^{\prime}>0$ then there exists a set $O^{\prime}$ in (1)' such that $M^{\prime} \subseteq O^{\prime}$ and $\mu^{\prime} O^{\prime} \leqq \mu^{\prime} M^{\prime}+\zeta^{\prime}$.

Note that $H 7^{*}$ implies $H 7$.

Definition. An element $O_{0}^{\prime}$ in $\mathbb{D}^{\prime}$ is said to be of type $Y^{\prime}$ if it is one of a countable number of pairwise disjoint sets $0 !, i \geqq 0$, in $\mathbb{D D}^{\prime}$ ' for which there exist two subsets $Y$ ' and $Z^{\prime}$ of S' such that

i) $\underline{S}^{\prime}=Y^{\prime} \cup Z^{\prime} \cup U_{i \geq 0^{0}}$;

ii) $\mathrm{T}^{-I_{\mathrm{Y}^{\prime}}}$ is in $\underline{\mathrm{M}}$ with $\mu \mathrm{T}^{-1} \mathrm{Y}^{\prime}=0$;

iii) $Z^{\prime}$ is in $\underline{M}^{\prime}$ with $\mu^{\prime} \mathrm{Z}^{\prime}=0$.

Definition. A set $M^{\prime}$ in $M^{\prime}$ is said to be of type $2^{\prime}$ if there is a sequence of sets $O_{j}^{\prime}$ in $\mathbb{D}^{\prime}$ such that $O_{j}^{\prime}$ is of type $Y^{\prime}$, $j \geqq 1, \quad 0: \subseteq 0_{j+1}^{\prime}$ for $j \geqq 1$, and $M^{\prime}=U_{j}$ 
H8. If $B^{\prime}$ is a set in $B^{\prime}$ there are a sequence of sets $\mathrm{M}$ in $M^{\prime}$ and two sets $U^{\prime}$ and $V^{\prime}$ in $M^{\prime}$ such that $M_{j}^{\prime}$ is of type $z^{\prime}$ for $j \geqq 1, M_{j} \supseteq M_{j+1}^{\prime}$ for $j \geqq I, \mu^{\prime} U^{\prime}=0=\mu^{\prime} V^{\prime}$, and $n_{j}^{\prime} U U^{\prime}=B^{\prime} U^{\prime} V^{\prime}$

Definition. Let $W^{\prime}$ be a non-negative function defined on S'XD. W' is termed a weight function for $T$ if the following four conditions are satisfied:

i) If $D$ is in $D$ and if $s^{\prime}$ is in $C^{\prime T D}$ then $W^{\prime}\left(s^{\prime}, D\right)=0$.

ii) If a set $D$ contains a countable number of pairwise disjoint sets $D_{i}$ then $\Sigma W^{\prime}\left(s^{\prime}, D_{i}\right) \leqq W^{\prime}\left(s^{\prime}, D\right)$ for every $s^{\prime}$ in $\underline{S}^{\prime}$

iii) If a set $D$ is the union of a countable number of sets $D_{j}$ such that $D_{j} \subseteq D_{j+1}$ for every $j$ then $\lim _{j} W^{\prime}\left(s^{\prime}, D_{j}\right)=W^{\prime}\left(s^{\prime}, D\right)$ for every $s^{\prime}$ in $\underline{s}^{\prime} \cdot$

iv) For each $D$ in $D$ the function $W^{\prime}\left(s^{\prime}, D\right), s^{\prime} \in \underline{S}^{\prime}$, is M'-measurable.

H9. W' is a weight function for T. (Thus W' is a non-. negative function defined on $\underline{S} \times \underline{D}$ satisfying conditions $i)$ - iv) of the definition just given.)

Remark. The remaining hypotheses are never considered unless $\mathrm{Hl}$ - H8 are already in effect.

H10. There exists a countable subset ${ }^{*} \underline{D}$ of $\underline{D}$ which contains the empty set $\varnothing$ and which has the following property.

Given a countable sequence of sets $D_{j}, j \geqq 0$, in $D$ such that 
$D_{0} \subseteq U_{j \geqq l} D_{j}$ then for every integer $j \geqq 0$ there exists a countable sequence of sets ${ }^{*} D_{j k}$ in ${ }^{*} D$ satisfying these conditions:

i) ${ }^{*} D_{\mathrm{Ok}} \leqq{ }^{*} D_{0} \mathbf{k + 1}$ for every $\mathbf{k}$ and $\underset{\mathbf{k}}{U^{*} D_{\mathrm{O}}}=\mathrm{D}_{\mathrm{O}}$;

ii) ${ }^{*} D_{O k} \subseteq U_{j \geqq 1}{ }^{*} D_{j k}$ for every $k,{ }^{*} D_{j k} \subseteq D_{j}$ for $j \geqq 1$ and $k \geqq 1$;

iii) for every positive integer $k$, there exists a positive integer $j_{k}$, depending on $k$, such that ${ }^{*} D_{j k}=\varnothing$ when $j>j_{k}$.

HII. If $s_{1}$ and $s_{2}$ are points in $\underline{S}$ for which there is a set $D_{0}$ in $D$ such that $s_{1} \in D_{0}$ and $s_{2} \& D_{0}$ then there are sets $D_{1}$ and $D_{2}$ in $D$ such that $s_{1} \in D_{1}, s_{2} \in D_{2}$, and $D_{1} \cap D_{2}=\varnothing$.

Definition. Set

$$
S=\{S \subseteq \underline{S}: S=\cap D, s \in D \in \underline{D}, \text { for some } s \text { in } \underline{S}\} \text {. }
$$

H12. For each $S$ in 5 the set TS consists of a single point in $\underline{S}^{\prime}$

Definition. Set

$$
\varepsilon=\{E \subseteq \underline{S}: \text { If } S \in S \text { and } S \cap E \neq \varnothing \text { then } S \subseteq E\}
$$

Definition. Assume HI - H8, HII, and Hl2. Define $S_{s^{\prime}}=\left\{S \in S: T S=s^{\prime}\right\}, s^{\prime} \in \underline{S}^{\prime}$. Let $w$ be a non-negative function defined on 5. Define a function

$$
W_{*}\left(s^{\prime}, D, w\right),\left(s^{\prime}, D\right) \in \underline{S}^{\prime} \times \underline{D},
$$

by 


$$
w_{*}\left(s^{\prime}, D, w\right)=\Sigma w S, s \in E_{s^{\prime}} \text { and } S \subseteq D,\left(s^{\prime}, D\right) \in \underline{S}^{\prime} \times \underline{D} ;
$$

here, the expression $\Sigma w S, S \in S_{s}$, and $S \subseteq D$, stands for the supremum of the set of numbers

$$
\left[0, \sum_{k=1}^{p} w s_{k}: s_{k} \in 5_{s^{\prime}}, s_{k} \subseteq D\right\} .
$$

H13. W' is a weight function for $T$ for which there exists a non-negative function w defined on 5 such that

$$
W^{\prime}\left(s^{\prime}, D\right)=W_{*}^{\prime}\left(s^{\prime}, D, W\right),\left(s^{\prime}, D\right) \in \underline{s^{\prime}} \times \underline{D} .
$$

H14. Assume H9. There exists a positive real number $t$ such that $W^{\prime}\left(s^{\prime}, D\right) \geqq t$ whenever $\left(s^{\prime}, D\right)$ is an element in $\underline{S}^{\prime} \times \underline{D}$ for which $W^{\prime}\left(s^{\prime}, D\right)>0$

H15. There exists a countable subset $\underline{D}^{* *}$ of $\underline{D}$ having the following properties:

i) For each $S$ in $S$ and $D$ in $\underline{D}$ such that $S \subseteq D$ there is a set $\dot{D}^{* *}$ in $D^{* *}$ such that $S \subseteq D^{* *} \subseteq D$.

ii) If for $s^{\prime}$ in $\underline{S}^{\prime}$ and $D$ in $\underline{D}$ there is a sequence of sets $D_{j}^{* *}$ in $\underline{D}^{* *}$ such that $T^{-1} s^{\prime} \cap D \subseteq U D_{j}^{* *}$ then there is also a sequence of sets $D_{l}^{*}$ in $D^{* *}$ such that $T^{-1} s^{\prime} \cap U D_{i}^{*}=\varnothing$ and $D \subseteq U_{j} D_{j}^{* *} U U_{i} D_{2}^{*}$

Remark. The following statement is denoted by HI5i for rather obvious reasons.

H15i. There exists a countable subset $\underline{D}^{* *}$ of $\underline{D}$ containing $\underline{S}$ and having the property that for each $S$ in 5 and $D$ in $D$ for 
which $S \subseteq D$ there is a set $D^{* *}$ in $\underline{D}^{* *}$ such that $S \subseteq D^{* *} \subseteq D^{*}$

I.2.2. The hypotheses HI - HI5 are introduced in [2]. Four additional hypotheses are introduced in this paper. They are denoted by $\mathrm{HCl}-\mathrm{HC} 4$.

HCl. $\underline{B}$ is a $\sigma-f i e l d$ of subsets of $\underline{S}$ for which $D \subseteq \underline{B} \subseteq \underline{M}$,

$\mathrm{TB}_{\mathrm{B}} \subseteq \underline{M}^{\prime}$, and $\mathrm{T}^{-1} \underline{\mathrm{B}}^{\prime} \subseteq \underline{\mathrm{B}}$.

HC2. For every $D$ in $D$ there is a countable sequence of sets $D_{i}$ in $D$ such that $D_{i} \subseteq D_{i+1}$ for every $i \geqq 1, D=U D_{i}$, and such that if $D^{j}, j \geqq 1$, is a countable number of sets in $\underline{D}$ such that $D \subseteq U{ }_{j \geqq I} D^{j}$, then for every positive integer $i$ there exists a positive integer $J(i)$ such that $D_{i} \subseteq U_{j=1}^{J(i)} D^{j}$.

HC3. D contains a countable subfamily $\underline{D}_{\text {\# }}$ with the property that every set $D$ in $\underline{D}$ is the union of some sequence of sets $D_{\# j}$, $j \geqq I$ in $\underline{D}_{\#}$ for which $D_{\# \mathbf{j}} \subseteq D_{\# j+1}, j \geqq 1$. HC4. $\underline{D}$ contains a countable subfamily $\underline{D}_{*}$ having the following properties:

i) For every $D$ in $\underline{D}$ there is a countable sequence of sets $D_{*_{i}}, i \geqq 1$, in $\underline{D}_{*}$ for which $D=U D_{*_{i}}$ and $D_{* i} \subseteq D_{*} i+1, i \geqq 1$.

ii) If $D$ in $D$ contains a finite number of sets $D_{* I}$, 


$$
\begin{aligned}
& \ldots . D_{* n} \text { in } \underline{D}_{*} \text { then there exists a set } D_{*} \text { in } \underline{D}_{*} \\
& \text { such that } U_{i=1}^{n} D_{* i} \subseteq D_{*} \subseteq D .
\end{aligned}
$$

\section{I.3 Basic definitions from [2]}

Throughout this section the standard hypotheses HI - H8 are always assumed.

I.3.1. Definition. Let $W^{\prime}$ be weight function for T. The function $W^{\prime}\left(s^{\prime}, D\right), s^{\prime} \in \underline{S}^{\prime}$, is $\underline{M}^{\prime}-m e a s u r a b l e$ for each $D$ in D. Set

$$
W D=\int_{S^{\prime}} W^{\prime}\left(s^{\prime}, D\right) d \mu^{\prime}, D \in \underline{D} .
$$

WD is termed the weight attached to $D$ by W'.

I.3.2. Definition. Let $W^{\prime}$ be a weight function for T. The transformation $T$ is said to be of bounded variation with respect to the attached weights W - briefly, T is BVW - if $W_{\underline{S}}=\int_{\underline{S}^{\prime}} W^{\prime}\left(s^{\prime}, \underline{S}\right) \mathrm{d} \mu^{\prime}$ is a finite real number.

I.3.3. Definition. Let $W$ ' be a weight function for T. Let $f(s), s \in \underline{S}$, be a non-negative M-measurable function such that $\int_{D} f(s) d \mu \leqq W D$ for every $D$ in $D$. Such a function $f$ is termed a lower bound function for the weights $W$ - briefly, a I.b.f.W.

I.3.4. Definition. Let $W$ ' be a weight function for $T$. The transformation I is said to be absolutely continuous with respect to the attached weights W-briefly, T is ACW - if $T$ 
is $\mathrm{BVW}$ and if there exists a non-negative M-measurable function $f$ defined on $\underline{S}$ which is a l.b.f.W and for which $\int_{\underline{S}} f(s) d \mu=W \underline{S}$. Such a function $f$ is termed a greatest lower bound function for the weights W-briefly, a g.l.b.f.W.

I.3.5. Definition. Let $W$ ' be a weight function for $T$. Then $W$ - that is, the function which associates the weight WD to every $D$ in $\underline{D}$ - is over additive (on $\underline{D}$ ) if $W D_{0} \leqq \Sigma_{i \geq l} W D_{i}$ whenever $D_{i}, i \geqq 0$, is a countable number of sets in $\underline{D}$ for which $D_{0} \subseteq U_{i \geqq 1} D_{i}$

I.3.6. Definition. Let $W$ ' be a weight function for $T$. Then $W$ is absolutely continuous with respect to the measure $\mu$ if for every $M$ in $M$ for which $\mu M=0$ and for every $\varepsilon>0$ there exists a countable number of sets $D_{j}$ in $D$ such that $M \subseteq U D_{j}$ and $\Sigma W D_{j}<\varepsilon$

I.3.7. Remark. The sets $5, \varepsilon$, and $\subseteq_{B}$, were defined in Section I.2. Also, the expression $W^{\prime}\left(s^{\prime}, D, W\right)$ was defined in Section I.2.

I.3.8. Definition. Assume HIl and Hl2. Let $w$ be a nonnegative function defined on 5. Suppose that $W$ ' is a weight function for $T$. $W^{\prime}$ is said to be generated by $W$ if $W^{\prime}\left(s^{\prime}, D\right)=$ $=w_{*}^{\prime}\left(s^{\prime}, D, w\right),\left(s^{\prime}, D\right) \in \underline{s^{\prime}} \times \underline{D}$. 
I.4 Lemmas and theorems from [2]

We list those results from [2] which are used explicitly in this paper. The numbers preceding the results are those which appear with them in [2].

3.1. Lemma. Assume $H I$ - H9. Assume that $T$ is $B V W$ and that $f$ is a l.b.f.W such that $W \underline{S}=\int_{\underline{S}} f(s)$ d $\mu$. Then $W D=\int_{D} f(s)$ d $\mu$ for every $D$ in $\underline{D}$.

3.2. Lemma. Assume HI - H9. Assume that $f_{1}$ and $f_{2}$ are non-negative $\mu$-integrable functions defined on $\underline{S}$ for which $\int_{D} f_{1}(s) d \mu \leqq \int_{D} f_{2}(s) d \mu, D \in D$. Then $f_{1}(s) \leqq f_{2}(s)$ a.e. $\mu$ on $\underline{S}$.

4.1. Lemma. Assume HI - H9. Assume that T is ACW. Let $D$ be a set in $\underline{D}$ for which there are a countable number of pairwise disjoint sets $D_{i}$ in $\underline{D}$ and two subsets $E$ and $F$ of $\underline{S}$ satisfying the relations

$$
\begin{aligned}
& \text { i) } \cup D_{i} \cong D, D \cap C\left(U D_{i}\right)=E \cup F ; \\
& \text { ii) } E \in \underline{M}, \mu E=0 ; \\
& \text { iii) } T F \in \underline{M}{ }^{\prime}, \mu^{\prime} T F=0 .
\end{aligned}
$$

Then $W D=\Sigma W D_{i}$ and $W^{\prime}\left(s^{\prime}, D\right)=\Sigma W^{\prime}\left(s^{\prime}, D_{i}\right)$ a.e. $\mu^{\prime}$ on $\underline{s}^{\prime} \cdot$ 4.10. Theorem. Assume HI - H9. Assume that T is ACW. Assume also that $f$ is a g.l.b.f.W. Fix D in D. Suppose that

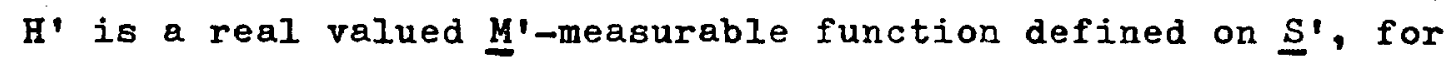
which either $H^{\prime}\left(s^{\prime}\right) W^{\prime}\left(s^{\prime}, D\right), s^{\prime} \in \underline{S}^{\prime}$, is $\mu^{\prime}$-integrable or $\operatorname{H} T(s) f(s), s \in D$, is $\mu$-integrable: then 
i) H'( $\left.s^{\prime}\right) W^{\prime}\left(s^{\prime}, D\right)$, $s^{\prime} \in \underline{S}^{\prime}$, is' $\mu^{\prime}$-integrable;

ii) $H \cdot T(s) f(s), s \in D$, is $\mu$-integrable;

iii) the transformation formula

$$
\int_{D} H^{\prime} T(s) f(s) d \mu=\int_{S^{\prime}} H^{\prime}\left(s^{\prime}\right) W^{\prime}\left(s^{\prime}, D\right) d \mu^{\prime}
$$

is valid.

5.11. Corollary. Assume HI - H9. Assume that $\mathrm{T}$ is BVW. Then a necessary and sufficient condition that $T$ be $A C W$ is that $W$ be over additive and absolutely continuous with respect to the measure $\mu$.

6.1. Lemma. Assume HI - H9. Assume that T is ACW. Then $W^{\prime}$ is an over additive function on $\underline{D}$ a.e. $\mu^{\prime}$ on $\underline{S}^{\prime}$ in the following sense: If $D_{0}$ is contained in the union of a countable number of sets $D_{j}, j \geqq 1$, then there exists a set $X^{\prime}=X^{\prime}\left\{D_{j}\right\}$ in $M^{\prime}$ such that $\mu^{\prime} X^{\prime}=0$ and such that $s^{\prime} \in C^{\prime} X^{\prime}$ implies $W^{\prime}\left(s^{\prime}, D_{0}\right) \leqq \Sigma_{j \geqq I^{\prime}} W^{\prime}\left(s^{\prime}, D_{j}\right)$. Also, if $T$ is BVW and $W^{\prime}\left(s^{\prime}, D\right)$ is.

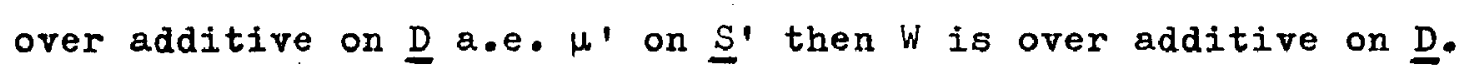
2.2. Lemma. Assume HI - H8. Assume also HIl. The sets in 5 constitute a partition of $\underline{S}$ - that is, the sets in 5 are nonempty, pairwise disjoint, and their union is $\underline{S}$. If $S$ in 5 and $D$ in $\underline{D}$ are such that $S \cap D \neq \varnothing$ then $S \subseteq D$. For every $S$ in $S$ we have $S=\cap D, S \subseteq D \in \underline{D}$.

7.7. Lemma. Assume HI - H8, HII, and HI2. Let $W$ be nonnegative function defined on 5 . Define $S^{+}=U S, S \in G$ and 
$w S>0$. Then, given $\left(s^{\prime}, D\right)$ in $\underline{S}^{\prime} \times \underline{D}, W_{*}^{\prime}\left(s^{\prime}, D, w\right)>0$ if and only if $8^{\prime}$ is in $T\left(S^{+} \cap D\right)$.

Remark. We list those results from 7.8 and 7.9 of [2] which we shall use.

Assume HI - H8, HII, and HI2. Let $w$ be a non-negative function defined on 5. Define $W^{\prime}$ by

$$
W^{\prime}\left(s^{\prime}, D\right)=W_{*}^{\prime}\left(s^{\prime}, D, W\right), \quad\left(s^{\prime}, D\right) \in \underline{S^{\prime} \times D .}
$$

Then:

1) Suppose a set D in $\underline{D}$ contains a countable number of pairwise disjoint sets $D_{i}$ in $D_{\text {. Then }} \Sigma W^{\prime}\left(s^{\prime}, D_{i}\right) \leqq$ $\leqq W^{\prime}\left(s^{\prime}, D\right)$ for every $s^{\prime}$ in $\underline{S}^{\prime} \cdot$

2) Suppose a set $D$ in $\underline{D}$ is contained in the union of a countable number of sets $D_{i}$ in $D$. Then $W^{\prime}\left(s^{\prime}, D\right) \leqq$ $\leqq \Sigma W^{\prime}\left(s^{\prime}, D_{i}\right)$ for every $s^{\prime}$ in $S^{\prime} \cdot$

3) Suppose a set $D$ in $D$ is the union of a sequence of $\operatorname{sets} D_{i}$ in $\underline{D}$ for which $D_{i} \cong D_{i+I^{*}}$ Then $W^{\prime}\left(s^{\prime}, D\right)=$ $=\lim _{i} W^{\prime}\left(s^{\prime}, D_{i}\right)$ for every $s^{\prime}$ in $\underline{S}^{\prime} \cdot$

4) W' is a weight function for $T$ if the function $W^{\prime}\left(s^{\prime}, D\right)$, $s^{\prime} \in \underline{S}^{\prime}$, is $\underline{M}^{\prime}-m e a s u r a b l e$ for every $D$ in $\underline{D}$.

2.24. Theorem. Assume HI - H8 and HII - HI3. Assume that $T$ is BVW. Then $T$ is ACW if and only if for every set $M$ in $M$ such that $M \subseteq U S, S \in S$, wS $>0$, and $\mu M=0$ it is true that $T M$ is in $\underline{M}^{\prime}$ and $\mu^{\prime} T M=0$.

8.2. Lemma. Assume $\mathrm{HI}$ - $\mathrm{H} 8$. If $\mathrm{D}_{\mathrm{k}}^{*}, \mathrm{k}=1, \ldots, \mathrm{p}$, is a 
finite sequence of sets in $\underline{D}$ then there exists a countable number of pairwise disjoint sets $D_{i}$ in $\underline{D}$ such that $n_{k=1}^{p} D_{k}^{*}=U D_{i}$.

8.3. Lemma. Assume $\mathrm{HI}-\mathrm{H} 8$ and HII. If $S$ is in 5 and if $D_{k}, k=1, \ldots, p$, is a finite sequence of sets in $D$ such that $S \subseteq n_{k=1}^{p} D_{k}$ then there is a set $D$ in $D$ such that $S \subseteq D \subseteq n_{k=1}^{p} D_{k}$

8.4. Lemma. Assume $H I-H 8$ and HII. If $S_{k}, k=1, \ldots, p$, is a finite sequence of distinct sets in 5 and if $D$ is a set in D such that $U_{k=1}^{p} S_{k} \subseteq D$ then there is a sequence of pairwise disjoint sets $D_{k}$ in $\underline{D}$ such that $S_{k} \subseteq D_{k} \subseteq D$ for $k=1, \ldots, p$. 8.5. Lemma. Assume HI - H9 and HII - H12. For each $S$ in 5 , TS is a point in $\underline{S}^{\prime}$. Define a non-negative function on 5 by $Q S=\inf \{W \cdot(T S, D): S \subseteq D \in \underline{D}\}, S \in S$.

Then

$$
W_{*}^{\prime}\left(s^{\prime}, D, \hat{w}\right) \leqq W^{\prime}\left(s^{\prime}, D\right),\left(s^{\prime}, D\right) \in \underline{S^{\prime}} X \underline{D} .
$$

8.9. Theorem. Assume HI - HI2, HI4, and H15. Assume that

$T$ is ACW. Define a non-negative function on 5 by

$$
\hat{W}=\inf (W \cdot(T S, D): S \subseteq D \in D\}, S \in S \text {. }
$$

Then there is a set $X^{\prime}$ in $\underline{M}^{\prime}$ such that $\mu^{\prime} X^{\prime}=0$ and $W_{*}^{\prime}\left(s^{\prime}, D, \hat{W}\right)=$ $=W^{\prime}\left(s^{\prime}, D\right)$ whenever $s^{\prime}$ is in $C^{\prime} X^{\prime}$ and $D$ is in $D$.

Remarks. The results of Section 9 of [2] are used in certain examples. 
Remark. The following result is a modification of Lemma 2.12 of [2], the proof of which is omitted.

Lemma. Assume HI - H8. Let $H^{\prime}$ be a non-negative $\underline{M}^{\prime}-$ measurable function defined on $\underline{S}^{\circ}$. There exists a non-negative $\underline{B}^{\prime}-$ measurable function $H_{0}^{\prime}$ defined on $\underline{S}^{\prime}$ such that $H^{\prime}=H_{0}^{\prime}$ a.e. $\mu^{\prime}$ on $\underline{S}^{\prime}$.

\section{I.5 Notational conventions}

I.5.1. Remarks. We shall often consider many weight functions simultaneously. It is therefore insufficient to denote all weight functions merely by W'. However, all weight functions will be denoted by the basic symbol W' together with some subscript or even possibly a superscript. Thus, W', W', W', and *W' denote weight functions (for $T$ ).

For each weight function there is the corresponding function which associates with each $D$ in $D$ its attached weight. This function will henceforth be denoted by the symbol for the corresponding weight function with the "prime" remored. Thus, $w, w_{0}, w_{\alpha}$, and $* W$ denote the weights induced by the weight functions $W^{\prime}, w_{o}^{\prime}, w_{\alpha}^{\prime}$, and *W'.

Occasionally, we write "Assume H9" rather than "Assume W' is a weight function for T." If we do write "Assume H9" it must be understood that $W^{\prime}$ (without other subscripts or superscripts) denotes the weight function thereby introduced. 
I.5.2. Remarks. The entities $\{\underline{S}, \underline{M}, \mu\},\left\{\underline{S}^{\prime}, \underline{M} \underline{M}^{\prime}, \mu^{\prime}\right\}, T$,

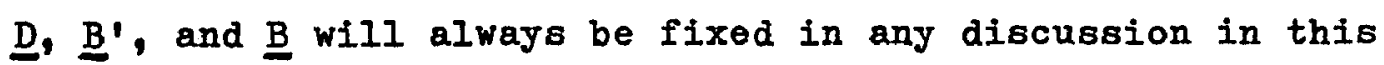
paper. Thus such symbols as $\underline{S}, \underline{S}, \mu, \underline{D}$, etc., always refer to the same thing. This remark is also valid for such symbols as $S, \varepsilon$, and $S_{s}, ;$ for these sets are defined entirely in terms of $\{\underline{S}, \underline{M}, \mu\},\left\{\underline{S}^{\prime}, \underline{M}^{\prime}, \mu^{\prime}\right\}, T, \underline{D}$, and $\underline{B}^{\prime} \cdot$

\section{I.5.3. Convention. The expression "AC" is used as an} abbreviation for both "absolutely continuous" and "absolute continuity".

I.5.4. Definitions. Assume $H I-H 8$. Let $W_{1}^{\prime}$ and $W_{2}^{\prime}$ be weight functions for $T$. We write $W_{1} \leqq W_{2}^{\prime}$ if and only if $W_{1}^{\prime}\left(s^{\prime}, D\right) \leqq W_{2}^{\prime}\left(s^{\prime}, D\right)$ for every $\left(s^{\prime}, D\right)$ in $\underline{S}^{\prime} \times \underline{D}$. The corresponding weights are denoted by $W_{1}$ and $w_{2} \cdot$ We write $w_{1} \leqq w_{2}$ if and only if $W_{1} D \leqq W_{2} D$ for every $D$ in $D$. Let $W_{0}$ be a third weight function for T. We write $W_{0}^{\prime}=W_{1}^{\prime}+W_{2}^{\prime}$ if and only if $W_{0}^{\prime}\left(s^{\prime}, D\right)=$ $=w_{1}^{\prime}\left(s^{\prime}, D\right)+w_{2}^{\prime}\left(s^{\prime}, D\right)$ for every $\left(s^{\prime}, D\right)$ in $\underline{s}^{\prime} \times \underline{D}$.

These definitions are in keeping with common usage.

I.5.5. Definition. Assume Hl - H8. Let $P\left(s^{\prime}, D\right)$ be a proposition defined for each $\left(8^{\prime}, D\right)$ in $\underline{S}^{\prime} X \underline{D}$. We say that $P\left(s^{\prime}, D\right)$ holds a.e. $\mu^{\prime}$ on $\underline{S}^{\prime}$ uniformly with respect to $\underline{D}$ if there is a set $X^{\prime}$ in $M^{\prime}$ such that $\mu^{\prime} X^{\prime}=0$ and such that $P\left(s^{\prime}, D\right)$ holds whenever s' is in $\mathrm{C}^{\prime} \mathrm{X}^{\prime}$ and $\mathrm{D}$ is in $\mathrm{D}$. The conclusion of 8.9 of 
[2] may be restated thus: Then $W^{\prime}\left(s^{\prime}, D, Q\right)=W^{\prime}\left(s^{\prime}, D\right)$ a.e. $\mu^{\prime}$ on $\underline{S}^{\prime}$ uniformly with respect to $\underline{D}$.

\section{I.6 Basic definitions}

Throughout this section HI - H8 are assumed.

I.6.1. Definition. Let $W$ ' be a non-negative function defined on $\underline{S} \times \underline{D}$. W' is said to be under additive on $\underline{D}$ if the following holds: Suppose a set $D$ in $\underline{D}$ contains a countable number of pairwise disjoint sets $D_{1}$ in $\underline{D}_{\text {; }}$ then $\Sigma W^{\prime}\left(B^{\prime}, D_{1}\right) \leqq W^{\prime}\left(s^{\prime}, D\right), s^{\prime} \in \underline{S}^{\prime} \cdot$

I.6.2. Remark. Often we say merely "W' is under additive" rather than " $W$ ' is under additive on $\underline{D}$ ".

I.6.3. Definition. Let $W$ ' be a non-negative function defined on $\underline{S} \times \underline{D}$. W' is said to be inner continuous (on D) if whenever a set $D$ in $D$ is the union of a sequence of sets $D_{i}$ in D for which $D_{i} \subseteq D_{1+I}, i \geqq I$, we have $W^{\prime}\left(s^{\prime}, D\right)=\lim W_{i} W^{\prime}\left(s^{\prime}, D_{1}\right)$, $\mathbf{B}^{\prime} \in \underline{S}^{\prime}$.

I.6.4. Remarks. Condition ii) for weight functions may be restated as follows: $W$ ' is under additive (on D). Condition iii) for weight functions may be restated as follows: $W$ is inner continuous (on D). 
I.6.5. Definition. Let $W$ ' be a non-negative function defined on $\underline{S}^{\prime} X \underline{D}$. $W$ ' is said to be almost under additive (on $\underline{D}$ ) if whenever a set $D$ in $\underline{D}$ contains a countable number of pairwise disjoint sets $D_{i}$ in $\underline{D}$ we have $\Sigma W^{\prime}\left(s^{\prime}, D_{i}\right) \leqq W^{\prime}\left(s^{\prime}, D\right)$ a.e. $\mu^{\prime}$ on $\underline{S}^{\prime}$.

1.6.6. Definition. Let $W$ ' be a non-negative function defined on $S^{\prime} X \underline{D}$. W' is said to be over additive (on D) if whenever a set $D$ is contained in the union of a countable number of sets $D_{i}$ in $D$ we have $W^{\prime}\left(s^{\prime}, D\right) \leqq \Sigma W^{\prime}\left(s^{\prime}, D_{i}\right), s^{\prime} \in \underline{S}^{\prime} \cdot$

I.6.7. Definition. Let $W \cdot$ be a non-negative function defined on $S^{\prime} X \underline{D}$. $W$ is said to be almost over additive (on $\underline{D}$ ) if whenever a set $D$ in $\underline{D}$ is contained in the union of a countable number of sets $D_{i}$ in $\underline{D}$ we have $W^{\prime}\left(s^{\prime}, D\right) \leqq \Sigma W^{\prime}\left(s^{\prime}, D_{i}\right)$ a.e. $H^{\prime}$ on $\underline{S}^{\prime}$.

I.6.8. Definition. Let $W$ ' be a non-negative function

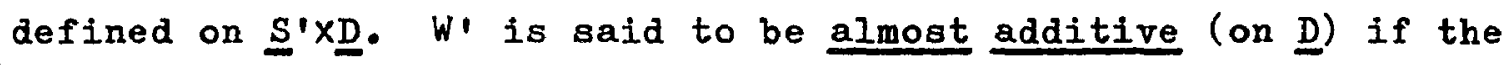
following holds: Suppose $D_{i}, i \geqq 0$, is a countable family of sets in $\underline{D}$ such that the sets $D_{i}, i \geqq 1$, are pairwise disjoint 1 and such that there exist subsets $Y$ and $Z$ of $S$ for which

$$
\begin{aligned}
& \text { i) } U_{i \gg I} D_{i} \cup Y \cup Z=D_{0} ; \\
& \text { ii) } Y \in \underline{M}, \mu Y=0 ; \\
& \text { iii) } T Z \in M \cdot, \mu \cdot T Z=0 .
\end{aligned}
$$

Then $W^{\prime}\left(B^{\prime}, D_{0}\right)=\Sigma_{i \geqq I^{\prime}} W^{\prime}\left(B^{\prime}, D_{i}\right)$ a.e. $\mu^{\prime}$ on $S^{\prime} \cdot$ 
I.6.9. Definition. Let $W^{\prime}$ and $W_{A}^{\prime}$ be weight functions for T. Assume that $W_{A}^{\prime} \leqq W^{\prime}$. Assume also that $T$ is $A_{C} W_{A}$ that is, assume that $T$ is absolutely continuous with respect to the weights $W_{A} D, D \in D$, induced by $W_{A} \cdot$ Then $W_{A}$ is said to be an AC part of $W^{\prime}$.

I.6.10. Definition. Let $W^{\prime}$ and $W_{A}$ be weight functions for T. Assume that $W_{A}^{\prime}$ is an AC part of $W^{\prime}$. Then $W_{A}^{\prime}$ is said to be a maximal $A C$ part of $W^{\prime}$ if $W_{A}^{\prime}=W_{a}^{\prime} a_{\bullet} e \cdot \mu^{\prime}$ on $\underline{S}^{\prime}$ uniformly with respect to $D$ whenever $W_{a}^{\prime}$ is any $A C$ part of $W^{\prime}$ for which $W_{A}^{\prime} \leqq W_{a}^{\prime}$ a.e. $\mu^{\prime}$ on $S^{\prime}$ uniformly with respect to $\underline{D}$.

I.6.11. Definition. Let $W^{\prime}$ and $W_{A}^{\prime}$ be weight functions for T. Assume that $W_{A}$ is an AC part of $W^{\prime}$. Then $W_{A}^{\prime}$ is said to be a strongly maximal $A C$ part of $W^{\prime}$ if $W_{a}^{\prime} \leqq W_{A}^{\prime} a_{\cdot e \cdot H^{\prime}}$ on $\underline{S}^{\prime}$ uniformly with respect to $D$ whenever $W_{a}^{\prime}$ is an $A C$ part of $W^{\prime} \cdot$

I.6.12. Definition. A weight function W' for T is said to be trivial if $W^{\prime}\left(s^{\prime}, \underline{S}\right)=0$ a.e. $\mu^{\prime}$ on $\underline{S}^{\prime}$.

I.6.13. Definition. A weight function $W '$ for $T$ is said to be singular if every AC part of $W^{\prime}$ is trivial.

I.6.14. Definition. Assume also HCl. A weight function W' for $T$ is said to be strongly singular if it is singular and if 
there exists a set $B_{0}$ in $\underline{B}$ such that $\mu B_{0}=0$ and $W^{\prime}\left(s^{\prime}, \underline{s}\right)=0$ a.e. $\mu^{\prime}$ on $\mathrm{C}^{\prime T B} \mathrm{O}^{\circ}$

I.6.15. Definition. Let $W$ ' be a weight function for T. Let $W_{A}$ and $W_{S}$ be weight functions for $T$. The ordered pair $\left(W_{A}^{\prime}, W_{S}^{\prime}\right)$ is said to be a Lebesgue-type decomposition for $W '$ if

1) $W^{\prime}=W_{A}^{\prime}+W_{S}^{\prime}$

2) $\mathrm{T}$ is $\mathrm{ACW}_{\mathrm{A}}$;

3) W' is singular.

$W_{A}^{\prime}$ is said to be the $A C$ component of the decomposition $\left(W_{A}^{\prime}, W_{S}\right)$. $W_{S}^{\prime}$ is said to be the singular component of the decomposition $\left(W_{A}^{\prime}, W_{S}^{\prime}\right)$

I.6.16. Definition. Assume HCl. Let $W$ ' be a weight function for T. Let $W_{A}$ and $W_{S}^{\prime}$ be weight functions for $T$. The ordered pair $\left(W_{A}^{\prime}, W_{S}^{\prime}\right)$ is said to be a strong Lebesgue-type decomposition for $W^{\prime}$ if it is a Lebesgue-type decomposition for $W^{\prime}$ and if $W_{S}^{\prime}$ is strongly singular.

\section{I.7 Preliminary lemmas}

Some preliminary lemmas are presented in this section. The results are varied and, to some extent, unrelated.

I.7.1. Lemma. Assume HI - H8. Let $W$ ' be a weight function for $T$ and let $H^{\prime}$ be a non-negative M'-measurable function defined 


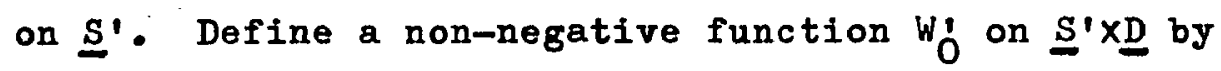

$$
W_{0}^{\prime}\left(s^{\prime}, D\right)=H^{\prime}\left(s^{\prime}\right) W^{\prime}\left(s^{\prime}, D\right),\left(s^{\prime}, D\right) \in \underline{s}^{\prime} \times \underline{D} .
$$

Then W' is a weight function for T.

Next suppose $W_{1}$ and $W_{2}^{\prime}$ are weight functions for T. Define $W_{0}^{\prime}$ by $W_{0}=W_{1}+W_{2}$. Then $W_{0}^{\prime}$ is a weight function for $T$. Finally suppose that $W_{i}^{\prime}$ and $W_{2}^{\prime}$ are weight functions for $T$ and suppose that $H_{1}^{\prime}$ and $H_{2}^{\prime}$ are non-negative real valued $\underline{M}^{\prime}-$ measurable functions defined on $\underline{S}^{\prime}$. Define $W_{0}^{\prime}$ on $\underline{S} \times \underline{D}$ by $W_{0}^{\prime}\left(s^{\prime}, D\right)=H_{1}^{\prime}\left(s^{\prime}\right) W_{1}^{\prime}\left(s^{\prime}, D\right)+H_{2}^{\prime}\left(s^{\prime}\right) W_{2}^{\prime}\left(s^{\prime}, D\right),\left(s^{\prime}, D\right) \in \underline{s}^{\prime} \times D$. Then $W_{0}^{\prime}$ is a weight function for $T . \quad$ (In particular, if $M^{\prime}$ is in $M^{\prime}$ and if we set $H_{j}^{\prime}=H_{M}^{\prime}$, and $H_{2}^{\prime}=H_{C}^{\prime} M$, then we see that the function $W_{0}^{\prime}$ defined by

$$
W_{0}^{\prime}\left(s^{\prime}, D\right)=\left\{\begin{array}{l}
W_{1}^{\prime}\left(s^{\prime}, D\right), \text { if }\left(s^{\prime}, D\right) \in M \cdot X D \\
W_{2}^{\prime}\left(s^{\prime}, D\right), \text { if }\left(s^{\prime}, D\right) \in C^{\prime} M \times X D
\end{array}\right.
$$

is a weight function for T.)

Remark. The last assertion of Lemma I.7.1 is often applied with $W_{i}$ chosen to be identically 0 . The set $M$ ' is quite often a set of $\mu^{\prime}$ measure 0 .

Lemma I.7.I is due to Reichelderfer. The proof is omitted.

I.2.2. Lemma. Assume HI - H8. Let $W_{0}^{\prime}, W_{1}^{\prime}$, and $W_{2}$ be weight functions for $T$ and suppose $W_{0}^{\prime}=W_{1}^{\prime}+W_{2}^{\prime} a_{\cdot} \cdot \mu^{\prime}$ on $\underline{S}^{\prime}$ 
uniformly with respect to $\underline{D}$. If $\mathrm{T}$ is $\mathrm{BVW}_{1}$ and $\mathrm{BVW}_{2}$ then $\mathrm{T}$ is $\mathrm{BVW}_{0}$ - If $\mathrm{I}$ is $\mathrm{ACW}_{1}$ and $\mathrm{T}$ is $\mathrm{ACW}_{2}$ then $\mathrm{T}$ is $\mathrm{ACW}_{0}$. If $\mathrm{T}$ is $\mathrm{ACW}_{0}$ and $\mathrm{T}$ is $\mathrm{ACW}_{1}$ then $\mathrm{T}$ is $\mathrm{ACW}_{2}$.

The straightforward proof is omitted. Lemma 3.2 of [2] is required for the proof of the last assertion.

I.7.3. Remarks. Assume HI - H9. Note that a strongly maximal AC part of $W^{\prime}$ is necessarily a maximal $A C$ part. If $W^{\prime}$ is strongly singular then it is singular. $W^{\prime}$ is trivial if and only if $W \underline{S}=0$. Every strong Lebesgue-type decomposition for W' is a Lebesgue-type decomposition for W'.

I.7.4. Lemma. Assume HI - H8. Suppose $W_{i}$ and $W_{2}$ are weight functions for $T$. Suppose that $W_{i}\left(s^{\prime}, D\right)=W_{2}^{\prime}\left(s^{\prime}, D\right)$ a.e. $\mu^{\prime}$ on $\underline{S}^{\prime}$ for every $D$ in $\underline{D}$. Then:

1) $w_{1}=w_{2}$.

2) $\mathrm{T}$ is $\mathrm{BVW}_{1}$ if and only if $\mathrm{T}$ is $\mathrm{BVW}_{2}$.

3) T is $\mathrm{ACW}_{1}$ if and only if $\mathrm{T}$ is $\mathrm{ACW}_{2}$.

4) $W_{i}$ is almost over (under) additive if and only if $W_{2}^{\prime}$ is almost over (under) additive.

5) $W_{1}$ is almost additive if and only if $W_{2}^{\prime}$ is almost additive.

Proof. The straightforward proof is omitted. 
I.7.5. Lemma. Assume HI - H8. Let $W_{i}$ and $W_{2}^{\prime}$ be weight functions for $T$. Assume that $W_{i}=W_{2}^{\prime}$ a.e. $\mu^{\prime}$ on $\underline{S}^{\prime}$ uniformly with respect to $\underline{D}$. Then statements 1) - 5) of Lemma 1.7 .4 are valid. Moreover, $W_{i}$ is singular if and only if $W_{2}^{\prime}$ is singular. Assume now that HCl also holds. Then $W_{i}$ is strongly singular if and only if $W_{2}^{\prime}$ is strongly singular.

Proof. The proof is omitted. The Remark following Lemma I.7.I is used.

I.7.6. Lemma. Assume HI - H8. Let $W^{\prime}, W_{1}$, and $W_{2}^{\prime}$ be weight functions for $T$ and suppose that $W_{i} \leqq W^{\prime}$ and $W_{2} \leqq W^{\prime}$. Assume also that $W_{i}^{\prime}=W_{i}^{\prime}$ a.e. $\mu^{\prime}$ on $\underline{S}^{\prime}$ uniformly with respect to $D$. Then $W_{i}$ is a maximal $A C$ part of $W^{\prime}$ if and only if $W_{2}$ is a maximal $A C$ part of $W \cdot W_{i}$ is a strongly maximal AC part of $W '$ if and only if $W_{2}^{\prime}$ is a strongly maximal $A C$ part of $W '$.

Proof. Obvious.

I.7.7. Lemma. Assume that $\mathrm{HI}-\mathrm{H} 8$ hold. Let W', W', and $W_{S}^{\prime}$ be weight functions for $T$ and suppose $W^{\prime}=W_{A}+W_{S}$. If $W_{A}$ is a maximal AC part of $W$ then $W_{S}^{\prime}$ is singular.

Proof. Assume that $W_{A}$ is a maximal AC part of $W^{\prime}$. We wish to prove that $W_{S}^{\prime}$ is singular. Thus let $W_{a}^{\prime}$ be an AC part of $W_{S}^{\prime} \cdot$ Hence $W_{a}^{\prime} \leqq W_{S}^{\prime}$ and $T$ is $A C W_{a}$. Define a non-negative 
function $w_{0}^{\prime}$ on $\underline{S}^{\prime} \times \underline{D}$ by $w_{0}^{\prime}\left(s^{\prime}, D\right)=w_{A}^{\prime}\left(s^{\prime}, D\right)+w_{a}\left(s^{\prime}, D\right)$, $\left(s^{\prime}, D\right) \in \underline{S} \times \underline{D}$. By Lemmas I.7.I and I.7.2 W' is a weight function for $T$ and $T$ is $A C W_{0}$. Moreover $W_{A}^{\prime} \leqq W_{O}^{\prime}=W_{A}^{\prime}+W_{Q}^{\prime} \leqq W_{A}^{\prime}+W_{S}^{\prime}=W^{\prime}$. But $W_{A}$ is a maximal AC part of $W^{\prime}$. Hence $W_{A}^{\prime}=W_{0}^{\prime} a_{\cdot} e \cdot H^{\prime}$ on $\underline{S}^{\prime}$ uniformly with respect to $\underline{D}$. In particular $W_{A}^{\prime}\left(s^{\prime}, \underline{S}\right)=W_{O}^{\prime}\left(s^{\prime}, \underline{S}\right)$ a.e. $\mu^{\prime}$ on $\underline{S}^{\prime}$. Consequently $W_{A} \underline{S}=W_{O} \underline{S}$. But also $W_{O} S=W_{A} \underline{S}+W_{a} \underline{S}$. As a result $W_{A} \underline{S}=W_{A} \underline{S}+W_{Q} \underline{S}$. Since $W_{A} \underline{S}$ is finite $W_{Q} \underline{S}=0$. Hence $W_{a}^{\prime}$ is trivial and so $W_{S}^{\prime}$ must be singular.

Remark. One may ask whether the conclusion of Lemma I.7.7 can be strengthened. In particular, can one show that $W_{A}$ must be a maximal $A C$ part of $W^{\prime}$ if $W_{S}^{\prime}$ is singular? This question is answered in Chapter II.

\section{I.7.8. Lemma. Assume $\mathrm{HI}$ - H8. Suppose $\mathrm{M}_{0}$ is an element}

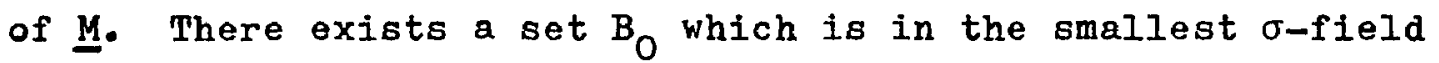
containing $\underline{D}$ and for which we have $\mathrm{M}_{\mathrm{O}} \cong \mathrm{B}_{0}$ and $\mu\left(\mathrm{B}_{0} \cap \mathrm{CM}_{0}\right)=0$. Proof. By $\mathrm{H}^{4} \underline{\mathrm{S}}$ can be written as the union of a countable number of sets ${ }_{*} D_{j}, j \geqq 1$, in $D$ for which $\mu_{*} D_{j}<\infty, j \geqq 1$, and such that the following holds: Given a set $M$ in $M$ with $M \subseteq{ }^{*} D_{j}$ for some $j$ and given $\varepsilon>0$, there is a countable number of pairwise disjoint sets $D_{i}$ in $\underline{D}$ such that $M \subseteq U D_{i}$ and $\Sigma \mu D_{i}<\mu M+\varepsilon$

Now let a set $M_{0}$ in $\underline{M}$ be given. Set $M_{j}=M_{0} \cap{ }_{*} D_{j}, j \geqq 1$. 
Then $M_{j}$ is in $M$ and $M_{f} \subseteq{ }_{*} D_{j}$ for each $j \cdot$ For every pair of positive integers $j$ and $n$ there is a countable number of pairwise disjoint sets $D_{n j i}, i \geqq 1$, in $\underline{D}$ such that $M_{j} \subseteq U_{1 \geqq I} D_{n j l}$ and $\Sigma_{i} \mu D_{n j i}<\mu M_{j}+\frac{I}{n}$. For each $j$, set $B_{j}=n_{n} U_{i} D_{n j i}$. Then, for each $j, B_{j}$ is in the smallest $\sigma$-field containing $\underline{D}, M_{j} \subseteq B_{j}$. and $\mu B_{j}=\mu M_{j}$. Indeed, since $\mu M_{j}<\infty$, it follows that $\mu\left(B_{j} \cap C M_{j}\right)=0, j \geqq I$. Now set $B_{0}=U_{j \geqq I} B_{j}$. Again, $B_{0}$ is in the smallest $\sigma-f i e l d$ containing D. Also $M_{0}=U_{j \geqq I} M_{j} \subseteq U_{j \geqq I} B_{j}=$ $=B_{0} \cdot$ And, since $B_{0} \cap \mathrm{CM}_{0} \subseteq U_{j \geqq I}\left(B_{j} \cap \mathrm{CM}_{j}\right)$, it follows that $\mu\left(B_{0} \cap \mathrm{CM}_{0}\right)=0$.

I.7.9. Lemma. Assume HI - H8, HIl, and HI2. Let $c_{1}, \ldots$, $c_{n}$ be positive real numbers and let $w_{1}, \ldots, w_{n}$ be non-negative functions defined on 5. Then $W_{*}^{\prime}\left(s^{\prime}, D, \Sigma_{i=1}^{n} c_{i} w_{i}\right)=\Sigma_{i=1}^{n} c_{i} W_{*}^{\prime}\left(s^{\prime}, D, w_{i}\right)$, $\left(s^{\prime}, D\right) \in \underline{S} \cdot \underline{D}$.

Proof. The result follows at once from (b) and (c) in Section I.1.2 of $[1]$.

I.7.10. Lemma. Assume HI - H8, HII, and HI2. Let $\left\{w_{m}\right\}_{m=I}^{\infty}$ be a sequence of non-negative functions defined on $\mathbb{S}$ for which $w_{m} \leqq w_{m+1}, m \geqq 1$. For each $S$ in $S$ the sequence $\left\{w_{m} S\right\}_{m=1}^{\infty}$ is convergent (perhaps to $\infty$ ). Define a non-negative function $w_{0}$ on 
5 by $w_{0} s=\lim _{m} w_{m} s, s \in 5$. Then the sequence

$\left\{W_{*}^{\prime}\left(B^{\prime}, D, W_{m}\right)\right\}_{m=1}^{\infty}$ is non-decreasing for every $\left(s^{\prime}, D\right)$ in $S^{\prime} X D$

and $\lim _{m} w_{*}^{\prime}\left(s^{\prime}, D, w_{m}\right)=w_{*}^{\prime}\left(s^{\prime}, D, w_{0}\right),\left(s^{\prime}, D\right) \in \underline{S}^{\prime} X \underline{D}$.

Proof. A simple direct proof can be supplied or one can appeal to (a) and (b) in section I.I.2 of [I].

I.7.11. Remarks. The standard hypotheses are HI - H15, H4*, $\mathrm{H} 7^{*}, \mathrm{H} 15 \mathrm{i}$, and $\mathrm{HCl}$ - HC4. At no point in this dissertation are all of these hypotheses assumed to hold. Specific mention of those assumed in any situation is always made. Some of the twenty-two standard hypotheses involve $\underline{D}$ alone. For example, HIO, H15i, and HC4 are concerned only with D. Among these there are some relationships. We list them, without proof:

1) Assume Hll. If HC3 holds so does HI5i.

2) If $\mathrm{HLO}$ is satisfied so is $\mathrm{HC} 3$.

3) If HC4 is satisfied so is HC3.

No attempt has been made to discover all of the relationships among the standard hypotheses. I) - 3) are merely the most obvious ones. They are sometimes used in the sequel without mention. 
CHAPTER II

THE MAIN DECOMPOSITION THEOREMS

The main objective of Chapter II is the statement and proof of the "General" and "Strong" decomposition theorems. However, other interesting properties of weight functions will also be established.

In Sections II.I through II. 6 we shall assume that $[\underline{S}, \underline{M}, \mu$ ) and $\left\{\underline{S}^{\prime}, \underline{M}^{\prime}, \mu^{\prime}\right\}$ are measure spaces, that $T$ is a single valued transformation from $\underline{S}$ onto $\underline{S}^{\prime}$, that $\underline{D}$ is a family of subsets of $\underline{S}$, and that $\underline{B}^{\prime}$ is a subfamily of $\underline{M}^{\prime}$ for which the standard hypotheses HI - H8 are satisfied. Throughout these sections then, $\{\underline{S}, \underline{M}, \mu\},\left\{\underline{S}^{\prime}, \underline{M}^{\prime}, \mu^{\prime}\right\}, T, \underline{D}$, and $\underline{B}^{\prime}$ remain fixed and hypotheses HI - H8 are always in force. Whenever any of the other standard hypotheses is used, it is mentioned explicitly. The notational conventions set forth in Chapter I will of course be observed faithfully.

II.I The difference of two weight functions

We shall not consider the difference of two arbitrary weight functions. Indeed such a difference is likely not to be non-negative; it could not possibly be a weight function: We shall however consider the difference of two welght functions 
satisfying certain conditions and show that this difference is essentially a weight function for $T$.

II.1.1. Definition. Suppose $\mathrm{E}$ is a subset of $\underline{S}$. The set $E$ is said to be of type $\gamma$ if there are a countable number of sets $D_{i}, i \geqq I$, in $D$ and subsets $Y$ and $Z$ of $S$ such that

$$
\begin{aligned}
& \text { i) } \mathbb{E} \cap D_{i}=\varnothing, i \geqq I ; \\
& \text { ii) } D_{i} \cap D_{j}=\varnothing, \text { if } i>j \geqq 1 ; \\
& \text { iii) } \underline{S}=E \cup \cup D_{i} \cup Y \cup Z ; \\
& \text { iv) } Y \in \underline{M}, \mu Y=0 ; \\
& \text { v) } T Z \in \underline{M}{ }^{\prime}, \mu \cdot T Z=0 .
\end{aligned}
$$

II.1.2. Remark. In Chapter I we defined the statement. "A set $D_{0}$ (in D) is of type $\gamma$ ". Examinations of that earlier definition and of the one just given reveal that a set $D_{0}$ in $D$ is of type $\gamma$ under the first definition if and only if it is of type $\gamma$ under the definition just given. Hence this latter definition is an extension of the earlier one.

II.1.3. Definition. Let $D_{i}, i \geqq 0$, be a countable family of sets in $\underline{D}$ and suppose that $Y$ and $Z$ are subsets of $\underline{S}$. Assume that

$$
\begin{aligned}
& \text { i) } D_{i} \cap D_{j}=\varnothing, \text { if } 1>j \geqq 1 ; \\
& \text { ii) } Y \in M, \mu Y=0 ; \\
& \text { iii) } T Z \in \underline{M}^{\prime}, \mu \cdot T Z=0 ; \\
& \text { iv) } D_{0}=U_{I \geqq I} D_{i} \cup Y \cup z .
\end{aligned}
$$


Then the triple $\left(\left\{D_{i}\right\}_{i \geq I}, Y, Z\right)$ is said to be a D-partition of $D_{0}$. The set $\left\{D_{i}\right\}_{i \gg 1}$ is termed the D-component of the D-partition $\left(\left\{D_{1}\right\}_{1 \geq 1}, Y, Z\right)$

Remark. Evidently a set $D$ in $D$ is of type $\gamma$ if and only if it occurs in the D-component of some D-partition of $\underline{S}$.

II.1.4. Definition. Let $\left\{D_{i}\right\}_{i=1}^{n}$ be a finite sequence of sets in $D$. The sequence $\left\{D_{i}\right\}_{i=1}^{n}$ is said to be of type $\gamma$ if there is some $\underline{D}$-partition of $\underline{S}$ the $\underline{D}$-component of which includes the set $\left\{D_{i}\right\}_{1=1}^{n}$. The remaining sets in the $D$-component of such a $\underline{D}-$ partition are said to form a $y$-complement of $\left\{D_{i}\right\}_{i=1}^{n}$.

Remark. If a sequence $\left(D_{i}\right\}_{i=1}^{n}$ is of type $\gamma$ then each $D_{i}$ $(i=1, \ldots, n)$ is of type $\gamma$.

II.I.5. Lemma. Suppose each of the subsets $E_{1}, \ldots, E_{n}$ of $\underline{S}$ is of type $\gamma$. Then $U_{i=1}^{n} E_{i}$ is of type $\gamma$.

Proof. We shall prove the result by induction on $n$. There is nothing to prove if $n=1$. It is convenient to prove the result for $n=2$.

Thus suppose $E_{1}$ and $E_{2}$ are sets of type $\gamma_{\bullet}$ For $i=1,2$, there are a sequence $\left[D_{1 k}\right\}_{k \geqq l}$ of sets in $D$ and subsets $Y_{i}$ and $z_{i}$ of $\underline{s}$ such that 


$$
\begin{aligned}
& \text { i) } \mathbb{E}_{i} \cap D_{i k}=\varnothing, k \geqq I ; \\
& \text { ii) } D_{i k_{1}} \cap D_{i k_{2}}=\varnothing \text {, if } k_{1}>k_{2} \geqq 1 ; \\
& \text { iii) } \underline{S}=E_{i} \cup U_{k} D_{i k} \cup Y_{i} \cup z_{i} ; \\
& \text { iv) } Y_{i} \in \underline{M}, \mu Y_{i}=0 ; \\
& \text { v) } \mathbb{T Z}_{i} \in \underline{M}^{\prime}, \mu \cdot T Z_{i}=0 .
\end{aligned}
$$

For every pair of positive integers $k_{1}$ and $k_{2}$ there is by $H_{4}$ a countable sequence $D_{k_{1} k_{2} m}$, m $\geqq 1$, of pairwise disjoint sets in $\underline{D}$ such that $D_{1 k_{1}} \cap D_{2 k_{2}}=U_{m \geq I} D_{k_{1} k_{2} m^{m}}$. It follows from ii) that the sets $D_{k_{1} k_{2} m}, k_{1} \geqq 1, k_{2} \geqq 1, m \geqq 1$, are pairwise disjoint. Next, note that, given any $\mathrm{D}_{k_{1} k_{2} m}$, we have

$$
\left(E_{1} \cup E_{2}\right) \cap D_{k_{1} k_{2} m} \subseteq\left(E_{1} \cap D_{1 k_{1}}\right) \cup\left(E_{2} \cap D_{2 k_{2}}\right)=\varnothing .
$$

Moreover the set $Y_{1} U Y_{2}$ is in $\underline{M}$ and $\mu\left(Y_{1} U Y_{2}\right)=0$. The set $T\left(z_{1} \cup z_{2}\right)$ is in $\underline{M}^{\prime}$ with

$$
\mu \cdot T\left(z_{1} \cup z_{2}\right) \leqq \mu \cdot T Z_{1}+\mu \cdot T Z_{2}=0 .
$$

Finally, it is easy to check that

$$
\left(E_{1} \cup E_{2}\right) \cup U_{k_{1}}, k_{2}, m_{k_{1} k_{2} m} \cup\left(Y_{1} \cup Y_{2}\right) \cup\left(z_{1} \cup z_{2}\right)=\underline{s} \text {. }
$$

The sets $D_{k_{1} k_{2} m}, k_{1} \geqq 1, k_{2} \geqq 1$, m $\geqq 1$, and the subsets $Y_{1} \cup Y_{2}$ and $z_{1} \cup z_{2}$ of $\underline{S}$ satisfy the five conditions of Definition II.1.1. Hence the set $E_{1} \cup E_{2}$ is of type $\gamma_{0}$ We proceed to the induction step. Suppose the result is 
true whenever $n=k$. Let $E_{1}, \ldots, E_{k+1}$ be $k+1$ sets of type $\gamma \cdot$ By the induction hypothesis the set $E_{1} U \ldots U E_{k}$ is of type $\gamma$. The union of two sets of type $\gamma$ is of type $\gamma$ and 80

$$
E_{1} \cup \ldots U E_{k+1}=\left(E_{1} \cup \ldots U E_{k}\right) \cup E_{k+1}
$$

must be of type $Y$.

The proof is completed by an inductive argument. Lemma II.1.5 is due to Reichelderfer.

II.I.6. Lemma. Let $\left\{D_{i}\right\}_{i=1}^{n}$ be a finite sequence of sets in $\underline{D}$ consisting of pairwise disjoint sets. Suppose that $D_{i}$, $i=1, \ldots, n$, is of type $\gamma$. Then the sequence $\left\{D_{i}\right\}_{i=1}^{n}$ is of type $Y$.

Proof. By Lemma II.1.5 the set $U_{i=1}^{n} D_{i}$ is of type $\gamma \cdot$ Hence there exist a countable number of sets $D_{i}, i \geqq n+1$, in $\underline{D}$ and subsets $Y$ and $Z$ of $\underline{S}$ such that

$$
\begin{aligned}
& \text { i) } U_{k=1}^{n} D_{k} \cap D_{i}=\varnothing, i \geqq n+I ; \\
& \text { ii) } D_{i_{1}} \cap D_{i_{2}}=\varnothing \text {, if } i_{1}>i_{2} \geqq n+1 ; \\
& \text { iii) } \underline{S}=U_{k=1}^{n} D_{k} \cup U_{i \geqq n+1} D_{i} \cup Y \cup Z ; \\
& \text { iv) } Y \in \underline{M}, \mu Y=0 ; \\
& \text { v) } T Z \in \underline{M} \cdot, \mu \cdot T Z=0 .
\end{aligned}
$$

It follows from $i)-v$ ) and from Definition II.1.3 that the triple $\left(\left\{D_{i}\right\}_{i \geq I}, Y, Z\right)$ is a $\underline{D}$-partition of $\underline{S} \cdot\left\{D_{i}\right\}_{i=1}^{n}$ is surely 
a subset of the D-component $\left\{D_{i}\right\}_{i \geq 1}$ of this D-partition. According to Definition II.1.4, $\left\{D_{i}\right\}_{i=1}^{n}$ is of type $\gamma$.

II.1.7. Lemma. Let $\left\{D_{i}\right\}_{i=1}^{n}$ be a finite collection of pairwise disjoint sets in $\underline{D}$. There is, for $1=1, \ldots, n$, a sequence $\left\{D_{i j}\right\}_{j=1}^{\infty}$ of sets in $\underline{D}$ such that

i) $D_{i j} \cong D_{i j+1}$, if $l \leqq i \leqq n, j \geqq l$;

ii) the set $\left[D_{i j}\right\}_{i=1}^{n}$ is of type $\gamma, j \geqq I$;

iii) $D_{i}=U_{j \geqq 1} D_{i j}, I \leqq i \leqq n$.

Proof. For $i=1, \ldots, n$ there exists by 15 a sequence of sets $D_{i j}, j \geqq 1$, in $\underline{D}$ such that
a) $D_{i j} \subseteq D_{i j+1}, j \geqq 1$;
b) each $D_{i j}$ is of type $\gamma, j \geqq 1$;
c) $D_{i}=U_{j \geqq I} D_{i j} \cdot$

Because the $D_{i}$ 's are pairwise disjoint and because of $b$ ) and $c)$, it follows that, for every $j \geqq l$, the sequence $\left(D_{i j}\right\}_{i=1}^{n}$ is a subset of $\underline{D}$ consisting of pairwise disjoint sets of type $\gamma$. By Lemma II.1.6 the sequence $\left[D_{i j}\right\}_{i=1}^{n}$ must be of type $\gamma$; the desired conclusion follows from this statement and from a) and $c$ ). 
II.1.8. Lemma. Let $\left\{D_{i}\right\}_{i=1}^{I}$ be a finite collection of pairwise disjoint sets in $D$ each of which is of type $\gamma$. Suppose $D_{0}$ is in $\underline{D}$ and $U_{i=1}^{I} D_{i} \subseteq D_{0}$. Then there exists a $\underline{D}$-partition of

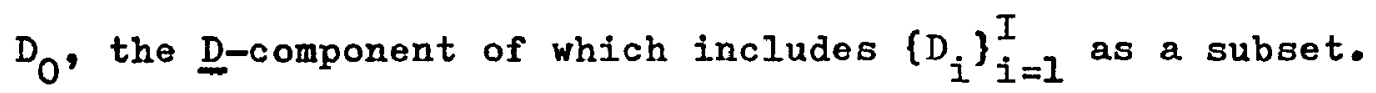
Proof. By Lemma II.1.6 the sequence $\left\{D_{i}\right\}_{i=1}^{I}$ is of type $Y$. There is a D-partition of $\underline{S}$ - say, $\left(\left(D_{k}^{\#}\right\}_{k \geq I}, Y_{\#}, Z_{\#}\right)$ - for which $\left(D_{1}\right)_{i=1}^{I} \subseteq\left[D_{k}^{\#}\right\}_{k \geqq 1}$. We may as well assume

$$
D_{i}=D_{i}^{\#}, i=1, \ldots, I \text {. }
$$

Thus the sets $D_{k}^{\#}, k \geqq I$, are pairwise disjoint, $Y_{\#}$ is in $\underline{M}$

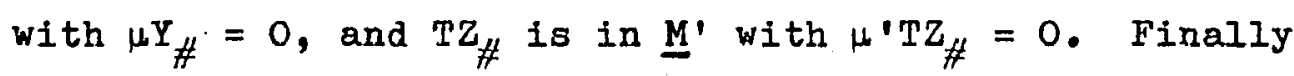
$\underline{S}=U_{k \geqq I} D_{k}^{\#} \cup Y_{\#} \cup Z_{\#} \cdot$ According to $H^{4}$, there is, for $k \geqq I+I$, a countable number of pairwise disjoint sets $D_{k j}, j \geqq 1$, in $\underline{D}$ such that $D_{0} \cap D_{k}^{\#}=U_{j \geqq I} D_{k j}$. Set $Y=Y_{\#} \cap D_{0}$ and $Z=Z_{\#} \cap D_{0}$. We now assert that the triple

$$
\left(\left\{D_{i}\right\}_{i=1}^{I} \cup\left\{D_{k j}\right\}_{k \geqq I+1, j \geqq 1}, Y, Z\right)
$$

is a D-partition of $\mathrm{D}_{\mathrm{O}^{*}}$ According to Definition II.1.3, there are four conditions to check. It is easy to see that the sets 
In the family $\left(D_{i}\right)_{i=1}^{I} U\left(D_{k j}\right\}_{k \geqq I+1, J \geqq I}$ are pairwise disjoint. Observe that

$$
\begin{aligned}
& U_{i=1}^{I} D_{i} \cup U_{k, j} D_{k j} \cup Y \cup z= \\
&=\left(D_{0} \cap U_{i=1}^{I} D_{i}\right) \cup U_{k \geqq I+1}\left(D_{0} \cap D_{k}^{\#}\right) \cup\left(D_{0} \cap Y_{\#}\right) \cup\left(D_{0} \cap z_{\#}\right)= \\
&=D_{0} \cap \underline{S}=D_{0} .
\end{aligned}
$$

Moreover, $Y$ is in $\underline{M}$ with $\mu Y=0$ and $T Z$ is in $\underline{M}^{\prime}$ with $\mu$ 'TZ $=0$. Hence $\left(\left\{D_{i}\right\}_{i=I}^{I} \cup\left\{D_{k j}\right\}_{k \gg I+I, j \geqq I}, Y, Z\right)$ is indeed a D-partition for $D_{0}$; its $\underline{D}-c$ omponent does include the sequence $\left\{D_{i}\right\}_{i=1}^{I}$.

II.1.9. Lemma. Suppose $W^{\prime}$ and $W_{A}^{\prime}$ are weight functions for $T$ and suppose that $W_{A}$ is a finite valued $A C$ part of 'W'. Define a non-negative function $W_{\Delta}^{\prime}$ on $\underline{S}^{\prime} X \underline{D}$ by

$$
W_{\Delta}\left(s^{\prime}, D\right)=W^{\prime}\left(s^{\prime}, D\right)-W_{A}^{\prime}\left(s^{\prime}, D\right),\left(s^{\prime}, D\right) \in \underline{s^{\prime}} X \underline{D} .
$$

Then $w_{\Delta}$ satisfies the standard conditions $\left.\left.i\right), i i i\right)$, and $\left.i v\right)$ of H9 and is almost under additive.

Proof. First we check the standard conditions i), iii), and $i v)$. Thus if $D$ is in $\underline{D}$ and $s^{\prime}$ is in C'TD then

$$
W_{\Delta}\left(s^{\prime}, D\right)=W^{\prime}\left(s^{\prime}, D\right)-W_{A}^{\prime}\left(s^{\prime}, D\right)=0-0=0 \text {. }
$$

Next, suppose that $D$ is the union of a sequence of sets $D_{1}$, $i \geqq 1$, in $\underline{D}$ for which $D_{i} \subseteq D_{i+1}$, $i \geqq 1$. Fix s' in $\underline{S}^{\prime} \cdot$ We must show that $\lim { }_{i} W_{\Delta}\left(s^{\prime}, D_{i}\right)$ exists and equals $W_{\Delta}^{\prime}\left(s^{\prime}, D\right)$. Indeed 
since $11 m_{1} W_{A}^{\prime}\left(s^{\prime}, D_{i}\right)=W_{A}^{\prime}\left(s^{\prime}, D\right)$ is finite it follows at once that

$$
\begin{aligned}
\lim w_{i} W_{\Delta}\left(s^{\prime}, D_{i}\right) & =\operatorname{Iim_{i}} W^{\prime}\left(s^{\prime}, D_{i}\right)-\lim w_{A}^{\prime}\left(s^{\prime}, D_{i}\right) \\
& =W^{\prime}\left(s^{\prime}, D\right)-W_{A}^{\prime}\left(s^{\prime}, D\right) \\
& =W_{\Delta}^{\prime}\left(s^{\prime}, D\right) .
\end{aligned}
$$

Finally, for a fixed $D$ in $\underline{D}$, the function $w_{\Delta}^{\prime}\left(s^{\prime}, D\right), \sigma^{\prime} \in \underline{S}^{\prime}$, is the difference of two $\underline{M}^{\prime}$-measurable functions $W^{\prime}\left(s^{\prime}, D\right), s^{\prime} \in \underline{S}^{\prime}$, and $W_{A}^{\prime}\left(s^{\prime}, D\right), s^{\prime} \in \underline{S}^{\prime} ;$ hence it is $\underline{M}^{\prime}$-measurable.

Now we prove that the function $w_{\Delta}$ is almost under additive. Assume that $\left\{D_{i}\right\}_{i=0}^{n}$ is a finite subset of $D$ for which the sets $D_{1}, i=1, \ldots, n$, are pairwise disjoint and $U_{i=1}^{n} D_{i} \cong D_{0}$. Then $\left\{D_{i}\right\}_{i=1}^{n}$ is a finite subset of $\underline{D}$ consisting of pairwise disjoint sets. By Lemma II.I.7, there is, for $I \leqq i \leqq n$, a sequence of sets $D_{i j}, j \geqq 1$, in $\underline{D}$ such that

$$
\begin{aligned}
& \text { i) } D_{i j} \subseteq D_{i} j+1, I \leqq 1 \leqq n \text { and } j \geqq 1 ; \\
& \text { ii) the set }\left\{D_{i j}\right\}_{i=1}^{n} \text { is of type } \gamma, j \geqq I ; \\
& \text { iii) } D_{i}=U_{j \geqq I} D_{i j}, I \leqq i \leqq n \text {. }
\end{aligned}
$$

Fix $j \geqq 1$; by Lemma II.1.8 the set $\left\{D_{i j}\right\}_{i=1}^{n}$ is a subset of the D-component of some D-partition $\left(\left\{D_{i}^{j}\right\}_{i \geqq I}, Y_{j}, Z_{j}\right)$ of $D_{0}$. We shall assume 


$$
D_{i j}=D_{i}^{j}, \quad 1 \leqq i \leqq n, \quad j \geqq 1 \text {. }
$$

We have

(1)

$$
U_{i \geqq 1} D_{i}^{j} \subseteq D_{0}
$$

Using (1) and the under additivity of $W^{\prime}$, we obtain

$$
\begin{aligned}
& w^{\prime}\left(s^{\prime}, D_{0}\right) \geqq \sum_{i=1}^{n} W^{\prime}\left(s^{\prime}, D_{i j}\right)+\sum_{i \gg n+1} w^{\prime}\left(s^{\prime}, D_{i}^{j}\right), \\
& s^{\prime} \in \underline{s}^{\prime} .
\end{aligned}
$$

By Lemma 4.1 of [2] we obtain

$$
\begin{aligned}
& W_{A}^{\prime}\left(s^{\prime}, D_{0}\right)=\sum_{i=1}^{n} W_{A}^{\prime}\left(s^{\prime}, D_{i j}\right)+\sum_{i \geqq n+I} W_{A}^{\prime}\left(s^{\prime}, D_{i}^{j}\right) \\
& \text { a.e. } \mu^{\prime} \text { on } \underline{S}^{\prime} \text {. }
\end{aligned}
$$

From (2) and (3) it follows that

$$
\begin{aligned}
& w_{\dot{\Delta}}\left(s^{\prime}, D_{0}\right)= w^{\prime}\left(s^{\prime}, D_{0}\right)-w_{A}^{\prime}\left(s^{\prime}, D_{0}\right) \\
& \geqq \sum_{i=1}^{n}\left\{W^{\prime}\left(s^{\prime}, D_{i j}\right)-w_{A}^{\prime}\left(s^{\prime}, D_{i j}\right)\right\}+ \\
&+\sum_{i \geqq n+1}\left\{w^{\prime}\left(s^{\prime}, D_{i}^{j}\right)-w_{A}\left(s^{\prime}, D_{i}^{j}\right)\right\} \\
& \geqq \sum_{i=1}^{n}\left\{w^{\prime}\left(s^{\prime}, D_{i j}\right)-w_{A}\left(s^{\prime}, D_{i j}\right)\right\} \\
& \text { a.e. } w^{\prime} \text { on } \underline{s}^{\prime} \cdot
\end{aligned}
$$

In short 


$$
W_{\Delta}\left(s^{\prime}, D_{0}\right) \geqq \sum_{i=1}^{n} W_{\Delta}\left(s^{\prime}, D_{i j}\right) \text { a.e. } \mu^{\prime} \text { on } \underline{S}^{\prime}, j \geqq I \text {. }
$$

We have already proved that $W_{\Delta}$ is inner continuous; hence, in view of $i)$ and $i 1 i)$, it follows that $\lim _{j} w_{\Delta}\left(s^{\prime}, D_{i j}\right)=w_{\Delta}\left(s^{\prime}, D_{i}\right)$, $s^{\prime} \in \underline{s}^{\prime}, 1 \leqq i \leqq n \cdot$ Consequently

$$
\operatorname{Iim}_{j} \sum_{i=1}^{n} w_{\Delta}^{\prime}\left(s^{\prime}, D_{i j}\right)=\sum_{i=1}^{n} w_{\Delta}^{\prime}\left(s^{\prime}, D_{i}\right), s^{\prime} \in \underline{s}^{\prime} \cdot
$$

Because of (4) and (5) we have $\Sigma_{i=1}^{n} w_{\Delta}^{\prime}\left(s^{\prime}, D_{i}\right) \leqq W_{\Delta}^{\prime}\left(s^{\prime}, D_{0}\right)$ a.e. $\mu^{\prime}$ on $\underline{S}^{\prime}$. Hence $W_{\Delta}^{\prime}$ is almost under additive.

II.1.10. Remark. Because of Lemma II.1.9, the function $W_{\Delta}^{\prime}$ might be described as a "pseudo" weight function for T. OnIy the required under additivity is lacking. From the point of view of integration this property is effectively present in the almost under additivity of $W_{\Delta}^{\prime}$. We shall now show that, subject to an additional hypothesis concerning the family D, there does exist a weight function for $T$ which agrees with $W_{\Delta}^{\prime} a_{\bullet} \cdot \mu^{\prime}$ on S' uniformly with respect to $\underline{D}$.

II.1.11. Lemma. Assume HC4. Let $\left\{D_{i}\right\}_{i=0}^{I}$ be a finite subset of $\underline{D}$ for which the sets $D_{i}, I \leqq i \leqq I$, are pairwise disjoint and $U_{i=1}^{I} D_{i} \subseteq D_{0}$. For each $i=0,1, \ldots, I$, there is a sequence of sets $D_{i j}^{*}, j \geqq 1$, in $\underline{D}_{*}$ such that 
I) $D_{i j}^{*} \cong D_{i j+1}^{*}, 0 \leqq i \leqq I$ and $j \geqq 1$;

2) $U_{j \geqq I} D_{i j}^{*}=D_{i}, \quad 0 \leqq i \leqq I$;

3) $U_{i=1}^{I} D_{i j}^{*} \cong D_{0 j}^{*}, j \geqq 1$.

Proof. We shall construct the "double" sequence $\left\{D_{i j}^{*}\right\}_{1=0}^{I} \sum_{j=1}^{\infty}$ by mathematical induction on $j \cdot$ According to HC4, there is, for $0 \leqq i \leqq I$, a sequence of sets ${ }^{*} D_{i j}, j \geqq I$, in $\underline{D}_{*}$ such that ${ }^{*} D_{i j} \subseteq{ }^{*} D_{i} j+1, j \geqq I$, and $D_{i}=U_{j \geqq I}{ }^{*} D_{i j} \cdot$ According to $i$ ) of $\mathrm{HC}_{4}$, there exists a set $D_{01}^{*}$ in $\underline{D}_{*}$ such that

$U_{i=0}^{I}{ }^{*} D_{i I} \leqq D_{01}^{*} \leqq D_{0}$. Now set $D_{i I}^{*}={ }^{*} D_{i I}, I \leqq i \leqq I$. We must observe that we have

$$
\begin{aligned}
& \text { i1) } D_{i I}^{*}={ }^{*} D_{i I}, I \leqq i \leqq I ; \\
& \text { iii ) } U_{i=1}^{I} D_{i I}^{*} U^{*} D_{0 I} \leqq D_{O I}^{*} \subseteq D_{0} \cdot
\end{aligned}
$$

Now assume that the family $\left\{D_{i I}^{*}\right\}_{i=0}^{I}$ has been extended to a finite subset $\left\{D_{i j}^{*}\right\}_{i=0}^{I} \underset{j=1}{k}$ of $\underline{D}_{*}$ for which

$$
\begin{aligned}
& \left.i_{k}\right) \quad D_{i j}^{*} \leqq D_{i j+1}^{*}, \quad 0 \leqq i \leqq I, I \leqq j<k ; \\
& \left.i i_{k}\right) \quad D_{i j}^{*}={ }^{*} D_{i j}, \quad I \leqq i \leqq I, I \leqq j \leqq k ; \\
& \left.i i i_{k}\right) \quad U_{i=1}^{I} D_{i j}^{*} U^{*} D_{0 j} \subseteq D_{0 j}^{*} \leqq D_{0}, \quad I \leqq j \leqq k .
\end{aligned}
$$

We wish to extend the set $\left\{D_{i j}^{*}\right\}_{i=0}^{I} \underset{j=1}{k}$ to a larger subset $\left\{D_{i j}^{*}\right\}_{i=0}^{I} j_{j=1}^{k+1}$ of $\underline{D}_{*}$ for which $\left.\left.i_{k}\right), i i_{k}\right)$, and $\left.i i i_{k}\right)$ hold with $k$ 
replaced by $k+1$. In order to carry out this extension, we use ii) of $\mathrm{HC} 4$ again. Thus, since $U_{i=0}^{I}{ }^{*} D_{i k+1} \subseteq D_{0}$, there exists a set $D_{0 k+1}^{*}$ in $\underline{D}_{*}$ such that $D_{0 k}^{*} U U_{i=0}^{I} D_{i k+1}^{*} \leqq D_{0}^{*}{ }_{k+1} \subseteq D_{0}$. Set $D_{i k+1}^{*}={ }^{*} D_{i k+1}, 1 \leqq i \leqq I$. Conditions $\left.\left.i_{k+1}\right), i_{k+1}\right)$, and $\left.{ }^{i 1 i_{k+1}}\right)$ are evidently satisfied by the set $\left\{D_{i j}^{*}\right\}_{i=0}^{I}{ }_{j=1}^{k+1}$.

By induction we can then continue to extend the successive families $\left.\left\{D_{i j}^{*}\right\}\right\}_{i=0}^{I} \underset{j=1}{k}$ to obtain a subfamily $\left\{D_{i j}^{*}\right\}_{i=0}^{I}{ }_{j=1}^{\infty}$ of D. for which

$$
\begin{aligned}
& \text { i) } D_{i j}^{*} \subseteq D_{i j+1}^{*}, 0 \leqq i \leqq I, j \geqq 1 ; \\
& \text { ii) } D_{i j}^{*}={ }^{*} D_{i j}, I \leqq i \leqq I, j \geqq 1 ; \\
& \text { iii) }{ }^{*} D_{0 j} \cup\left(U_{i=1}^{I} D_{i j}^{*}\right) \subseteq D_{0 j}^{*} \subseteq D_{0}, j \geqq 1 .
\end{aligned}
$$

We assert that this family $\left\{D_{i j}^{*}\right\}_{i=0}^{I} \sum_{j=1}^{\infty}$ satisfies conditions 1), 2), and 3). Indeed condition 1) of the conclusion is i) above. Condition 3) is an immediate consequence of iii). Using ii), we observe that

$$
U_{j \geqq I} D_{i j}^{*}=U_{j \geqq I} D_{i j}=D_{i}, \quad I \leqq i \leqq I ;
$$

using iii), we note that $D_{0}=U_{j \geqq I}{ }^{*} D_{0 j} \subseteq U_{j \geqq I} D_{0 j}^{*} \subseteq D_{0} \cdot$ Hence the family $\left\{D_{i j}^{*}\right\}_{i=0}^{I} \infty_{j=1}^{\infty}$ also satisfies 2$)$.

II.1.12. Lemma. Assume HC4. Suppose $W^{\prime}$ is a non-negative function defined on $\underline{S}^{\prime} \times \underline{D}$. Suppose that $W^{\prime}$ is inner continuous 
and almost under additive. There exists a set $M^{\prime}$ in $M^{\prime}$ such that $H^{\prime} M^{\prime}=0$ and such that the following holds: Whenever a set $D$ in $\underline{D}$ contains a countable number of pairwise disjoint sets $D_{i}$ in $\underline{D}$ then $\Sigma_{i \geqq I^{\prime}}\left(s^{\prime}, D_{i}\right) \leqq W^{\prime}\left(s^{\prime}, D\right), s^{\prime} \in C^{\prime} M^{\prime} \cdot$

Proof. Denote by $N$ the collection of all finite sequences $\left(D_{i}^{*}\right\}_{i=0}^{n}$ of sets in $\underline{D}_{*}$ for which the sets $D_{i}^{*}, 1 \leqq i \leqq n$, are pairwise disjoint and $U_{i=1}^{n} D_{i}^{*} \subseteq D_{0^{*}}^{*}$ Since $\underline{D}_{*}$ is countable the collection $N$ is also countable. By assumption $W '$ is almost under additive. Hence, for every set $\left[D_{i}^{*}\right\}_{i=0}^{n}$ in $\mu$, there exists a set $M^{\prime}\left(\left(D_{i}^{*}\right\}_{i=0}^{n}\right)$ in $M^{\prime}$ such that $\mu^{\prime} M^{\prime}\left(\left\{D_{i}^{*}\right\}_{i=0}^{n}\right)=0$ and

$$
\sum_{i=1}^{n} w^{\prime}\left(s^{\prime}, D_{i}^{*}\right) \leqq W^{\prime}\left(s^{\prime}, D_{0}^{*}\right), s^{\prime} \in C^{\prime} M^{\prime}\left(\left\{D_{i}^{*}\right\}_{i=0}^{n}\right) .
$$

Define $M^{\prime}=U M^{\prime}\left(\left\{D_{i}^{*}\right\}_{i=0}^{n}\right),\left\{D_{i}^{*}\right\}_{i=0}^{n} \in N^{\prime}$. Because $N$ is countable, it follows that $M^{\prime}$ is in $M^{\prime}$ and $\mu^{\prime} M^{\prime}=0$. Furthermore

$$
\sum_{i=1}^{n} w^{\prime}\left(s^{\prime}, D_{i}^{*}\right) \leqq w^{\prime}\left(s^{\prime}, D_{0}^{*}\right), s^{\prime} \in C^{\prime} M^{\prime},\left\{D_{i}^{*}\right\}_{i=0}^{n} \in k .
$$

Now let $\left\{D_{i}\right\}_{i=0}^{I}$ be any finite sequence of sets in $\underline{D}$ for which the sets $D_{i}, 1 \leqq i \leqq I$, are pairwise disjoint and $U_{i=1}^{I} D_{i} \subseteq D_{0}$. By Lemma II.I.II there exists a double sequence $\left\{D_{i j}^{*}\right\}_{i=0}^{I} \sum_{j=1}^{\infty}$ of sets in $\underline{D}_{*}$ with the properties listed as 1$)$, 2), and 3) in the conclusion of Lemma II.1.11. The sets $D_{i j}^{*}$, 
$I \leqq i \leqq I$, are necessarily pairwise disjolnt for every $f \cdot$ Hence, in view of 3$),\left\{D_{i j}^{*}\right\}_{i=0}^{I}$ is in $N$ for $j \geqq 1$. According to (1),

$$
\sum_{i=1}^{I} W^{\prime}\left(s^{\prime}, D_{i j}^{*}\right) \leqq W^{\prime}\left(s^{\prime}, D_{0 j}^{*}\right), \quad s^{\prime} \in C^{\prime} M^{\prime}, j \geqq 1 .
$$

Using 1), 2), and the inner continuity of $W^{\prime}$, we obtain

$$
\lim _{j} W^{\prime}\left(s^{\prime}, D_{i j}^{*}\right)=W^{\prime}\left(s^{\prime}, D_{i}\right), 0 \leqq i \leqq I, s^{\prime} \in \underline{S}^{\prime} \cdot
$$

Hence the sequence $\left\{\Sigma_{i=1}^{I} W^{\prime}\left(s^{\prime}, D_{i j}^{*}\right)\right\}_{j=1}^{\infty}$ is convergent for every $s^{\prime}$ in $\underline{S}^{\prime}$ and, using (2) and (3), we obtain

$$
\begin{aligned}
W^{\prime}\left(s^{\prime}, D_{0}\right) & =\lim W_{j}\left(s^{\prime}, D_{O j}^{*}\right) \\
& \geqq \operatorname{Iim} \sum_{i=I}^{I} W^{\prime}\left(s^{\prime}, D_{i j}^{*}\right) \\
& \geqq \sum_{i=1}^{I} W^{\prime}\left(s^{\prime}, D_{i}\right)
\end{aligned}
$$

whenever $s^{\prime}$ is in C'M'; in brief

$$
\sum_{i=I}^{I} w^{\prime}\left(s^{\prime}, D_{i}\right) \leqq W^{\prime}\left(s^{\prime}, D_{0}\right), s^{\prime} \in C^{\prime} M^{\prime} .
$$

Expression (4) holds whenever $\left\{D_{i}\right\}_{i=0}^{I}$ is any finite sequence of sets in $\underline{D}$ for which the sets $D_{i}, I \leqq i \leqq I$, are pairwise disjoint and $U_{i=1}^{I} D_{i} \subseteq D_{0}$

Finally let $D$ be a set in $D$ which contains a countable number of pairwise disjoint sets $D_{i}, i \geqq 1$. By the result proved in the preceding paragraph, we have 


$$
\sum_{i=1}^{I} w^{\prime}\left(s^{\prime}, D_{i}\right) \leqq W^{\prime}\left(s^{\prime}, D\right), \quad s^{\prime} \in C^{\prime} M^{\prime},
$$

for every positive integer I. Hence

$$
\Sigma_{i \geqq 1} W^{\prime}\left(s^{\prime}, D_{i}\right) \leqq W^{\prime}\left(s^{\prime}, D\right), \quad s^{\prime} \in C^{\prime} M^{\prime} \cdot
$$

II.1.13. Theorem. Assume HC4. Assume that $W^{\prime}$ and $W_{A}$ are weight functions for $T$. Assume that $W_{A}$ is a finite valued $A C$

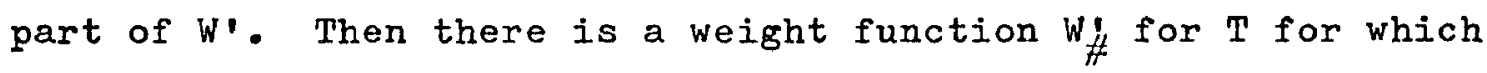
$W^{\prime} W^{\prime}=W_{A}^{\prime}+W_{A}$ a.e. $\mu^{\prime}$ on $S^{\prime}$ uniformly with respect to $D$.

Proof. Define the function $W_{\Delta}^{\prime}$ on the set $\underline{S}^{\prime} X \underline{D}$ by

$$
w_{\Delta}^{\prime}\left(s^{\prime}, D\right)=w^{\prime}\left(s^{\prime}, D\right)-w_{A}^{\prime}\left(s^{\prime}, D\right), \quad\left(s^{\prime}, D\right) \in \underline{s^{\prime}} \times \underline{D} .
$$

According to Lemma II.I.9, $W_{\Delta}^{\prime}$ satisfies conditions $\left.i\right)$, iii), and $i v)$ of $\mathrm{H} 9$ and is almost under additive. By Lemma II.I.12, there exists a set $M^{\prime}$ in $M^{\prime}$ such that $\mu^{\prime} M^{\prime}=0$ and such that the following holds: Given a set $D$ in $D$ which contains a countable number of pairwise disjoint sets $D_{i}$ in $\underline{D}$ then $\Sigma_{i} W^{\prime}\left(s^{\prime}, D_{i}\right) \leqq W^{\prime}\left(s^{\prime}, D\right)$ for every $s^{\prime}$ in C'M'.

Now define a non-negative function $W_{\#}$ on $\underline{S} \times \underline{D}$ by

$$
W_{\#}\left(s^{\prime}, D\right)=\left\{\begin{array}{cl}
W_{\Delta}^{\prime}\left(s^{\prime}, D\right) & \text { if } s^{\prime} \in C^{\prime} M^{\prime}, D \in \underline{D} \\
0 & \text { if } s^{\prime} \in M^{\prime}, D \in \underline{D}
\end{array} .\right.
$$

Observe that $W_{\#} \leqq W_{\Delta} \cdot$ We assert that $W_{\#}^{\prime}$ is a weight function for T. Condition $i)$ of $H 9$ follows from the facts that $W_{\#} \leqq w_{\Delta}^{\prime}$ and that $W_{\Delta}^{\prime}$ itself satisfies condition $\left.i\right)$ of 199 . The inner 
continuity of $w_{\#}$ follows from the inner continuity of $w_{\Delta} ;$ hence. $W_{\#}$ satisfies condition $\left.i i i\right)$ of $H 9$. Given D in $\underline{D}$, the function $W_{\#}\left(s^{\prime}, D\right), s^{\prime} \in \underline{S}^{\prime}$, is evidently $\underline{M}^{\prime}$-measurable; hence $W_{\#}$ satisfies condition $i \nabla)$ of H9. In order to show that $W_{\#}^{\prime}$ is a weight function for $T$, we now have only to show that it is under additive. Thus suppose a set $D$ in $D$ contains a countable number of pairwise disjoint sets $D_{i}$ in $\underline{D}$. In case $s^{\prime}$ is in $M$ ', we have $\Sigma_{i} W_{\#}^{\prime}\left(s^{\prime}, D_{i}\right)=0=W_{\#}^{\prime}\left(s^{\prime}, D\right) . \quad B u t$, in case $s^{\prime}$ is in $C^{\prime} M^{\prime}$, $\Sigma_{i} W_{\#}^{\prime}\left(s^{\prime}, D_{i}\right)=\Sigma_{i} W_{\Delta}^{\prime}\left(s^{\prime}, D_{i}\right) \leqq W_{\Delta}^{\prime}\left(s^{\prime}, D\right)=W_{\#}^{\prime}\left(s^{\prime}, D\right)$. Hence $W_{\#}^{\prime}$ is under additive. Hence $W_{\#}^{\prime}$ is a weight function for $T$. Finally, observe that

$$
\begin{aligned}
w^{\prime}\left(s^{\prime}, D\right) & =w_{A}^{\prime}\left(s^{\prime}, D\right)+w_{\Delta}^{\prime}\left(s^{\prime}, D\right) \\
& =w_{A}^{\prime}\left(s^{\prime}, D\right)+w_{\nexists f}^{\prime}\left(s^{\prime}, D\right)
\end{aligned}
$$

if $s^{\prime}$ is in $C^{\prime} M^{\prime}$ and $D$ is in $D$. Since $\mu^{\prime} M^{\prime}=0$, it follows that $W^{\prime}=W_{A}^{\prime}+W_{\#}^{\prime}$ a.e. $\mu^{\prime}$ on $\underline{S}^{\prime}$ uniformiy with respect to $\underline{D}$.

II.1.14. Theorem. Assume HC4. Let $W^{\prime}$ and $W_{a}^{\prime}$ be weight functions for $T$ and suppose that $W_{a}^{\prime}$ is an AC part of $W^{\prime}$. Then there exist weight functions $W_{A}^{\prime}$ and $W_{\#}^{\prime}$ for $T$ such that
(a) $W^{\prime}=W_{A}^{\prime}+W_{\#}$;
(b) $w_{A}^{\prime} \leqq w_{a}^{\prime} ;$ 
(c) $W_{A}^{\prime}=W_{a}^{\prime}$ a.e. $\mu^{\prime}$ on $\underline{S}^{\prime}$ uniformly with respect to $D_{i}$

(d) $\mathrm{T}$ is $^{2} \mathrm{ACW}_{\mathrm{A}}$.

Proof. By assumption $\mathrm{T}$ is $\mathrm{ACW}_{\mathrm{a}} \cdot$ Hence $\mathrm{T}$ is $\mathrm{BVW}{ }_{\mathrm{a}}$. As a result, $w_{a}^{\prime}\left(s^{\prime}, \underline{S}\right)<\infty$ a.e. $\mu^{\prime}$ on $\underline{S}^{\prime}$. Indeed, since $w_{a}^{\prime}$ is under additive, $W_{a}^{\prime}\left(s^{\prime}, D\right)<\infty$ a.e. $\mu^{\prime}$ on $\underline{S}^{\prime}$ uniformly with respect to D. There exists a set $M_{i}^{\prime}$ in $M^{\prime}$ such that $\mu^{\prime} M_{i}^{\prime}=0$ and $W_{a}^{\prime}\left(s^{\prime}, D\right)<$ $<\infty, s^{\prime} \in C^{\prime} M_{1}^{\prime}, D \in \underline{D}$. Define a non-negative function $W_{0}^{\prime}$ on $\underline{S} \cdot \underline{X}$ by

$$
W_{0}^{\prime}\left(s^{\prime}, D\right)=\left\{\begin{array}{c}
W_{a}^{\prime}\left(s^{\prime}, D\right), s^{\prime} \in C^{\prime} M_{l}^{\prime}, D \in \underline{D} \\
0, s^{\prime} \in M_{i}^{\prime}, D \in \underline{D}
\end{array} .\right.
$$

By Lemma I.7.1 $\mathrm{W}_{O}^{\prime}$ is a finite valued weight function for $T$ and $W_{0}^{\prime} \leqq W_{a}^{\prime} \leqq W^{\prime}$. Moreover, since $W_{o}^{\prime}=W_{a}^{\prime}$ a.e. $\mu^{\prime}$ on $S^{\prime}$ uniformly with respect to $\mathrm{D}$, it follows from Lemma $I .7 .5$ that $T$ is $\mathrm{ACW}_{0}$. The weight functions $W^{\prime}$ and $W_{0}^{\prime}$ (for $T$ ) satisfy the hypotheses of Theorem II.1.13. Hence there exists a weight function $W_{\square}$ for $T$ for which $W^{\prime}=W_{0}^{\prime}+W_{\dot{b}}^{\prime} a_{\cdot} e_{\bullet} \mu^{\prime}$ on $\underline{S}^{\prime}$ uniformly with respect to D. There is a set $M_{2}^{\prime}$ in $M^{\prime}$ such that $\mu^{\prime} M_{2}^{\prime}=0$ and

$$
W^{\prime}\left(s^{\prime}, D\right)=W_{0}^{\prime}\left(s^{\prime}, D\right)+W_{a^{\prime}}\left(s^{\prime}, D\right), \quad s^{\prime} \in C^{\prime} M_{2}^{\prime}, D \in \underline{D} .
$$

Define non-negative functions $w_{A}^{\prime}$ and $W_{\#}$ on $\underline{S}^{\prime} \times D$ by 


$$
W_{A}\left(s^{\prime}, D\right)=\left\{\begin{array}{c}
W_{a}^{\prime}\left(a^{\prime}, D\right), \text { if } s^{\prime} \in C^{\prime}\left(M_{i}^{\prime} U M_{2}^{\prime}\right), D \in \underline{D} \\
0, \text { if } s^{\prime} \in M_{I} \cup M_{2}^{\prime}, D \in \underline{D}
\end{array}\right.
$$

and

$$
w_{\#}\left(s^{\prime}, D\right)=\left\{\begin{array}{l}
w_{a^{\prime}}\left(s^{\prime}, D\right), \text { if } s^{\prime} \in C^{\prime}\left(M_{1}^{\prime} \cup M_{2}^{\prime}\right), D \in \underline{D} \\
w^{\prime}\left(s^{\prime}, D\right), \text { if } s^{\prime} \in M_{1}^{\prime} \cup M_{2}^{\prime}, D \in \underline{D}
\end{array} .\right.
$$

By Lemma I.7.1 both $W_{A}^{\prime}$ and $W_{\#}$ are weight functions for T.

We assert that the weight functions $W_{A}^{\prime}$ and $W_{\#}^{\prime}$ satisfy

conditions (a) - (d) of the conclusion. (a) follows from (1)

and (2). Conditions (b) and (c) are easily verified. Condition

(d) is an immediate consequence of Lemma I.7.5 and (c).

II.1.15. We prove a converse of Lemma I.7.7. One additional assumption is required.

Theorem. Assume HC4. Let $W^{\prime}, W_{A}^{\prime}$, and $W_{S}^{\prime}$ be weight functions for $T$ and suppose that $W^{i}=W_{A}^{\prime}+W_{S}^{\prime}$. Assume also that $T$ is $A_{A} W_{A}$ If $W_{S}^{\prime}$ is singular then $W_{A}^{\prime}$ is a maximal $A C$ part of $W !$.

Proof. Suppose $W_{a}^{\prime}$ is an AC part of $W^{\prime}$ for which $W_{A}^{\prime} \leqq W_{a}^{\prime}$ a.e. $\mu^{\prime}$ on $\underline{S}^{\prime}$ uniformly with respect to $\underline{D}$. There is a set $M_{0}^{\prime}$ in $\underline{M}^{\prime}$ such that $\mu^{\prime} M_{O}^{\prime}=0$ and $0 \leqq W_{A}^{\prime}\left(s^{\prime}, D\right) \leqq W_{a}^{\prime}\left(s^{\prime}, D\right)<\infty, s^{\prime} \in C^{\prime} M_{O}^{\prime}$, $D \in \underline{D}$. Define non-negative functions $W_{1}$ and $W_{2}$ on $\underline{S} \cdot \underline{X}$ by

$$
W_{i}^{\prime}\left(s^{\prime}, D\right)=\left\{\begin{array}{c}
W_{A}^{\prime}\left(s^{\prime}, D\right), s^{\prime} \in C^{\prime} M_{0}^{\prime}, D \in \underline{D} \\
0, s^{\prime} \in M_{0}^{\prime}, D \in \underline{D}
\end{array}\right.
$$

and 


$$
W_{2}^{\prime}\left(B^{\prime}, D\right)=\left\{\begin{array}{cl}
W_{a}^{\prime}\left(s^{\prime}, D\right), & s^{\prime} \in C^{\prime} M_{0}^{\prime}, D \in \underline{D} \\
0, & s^{\prime} \in M_{0}^{\prime}, D \in \underline{D}
\end{array}\right.
$$

By Lemma I.7.1 $W_{1}^{\prime}$ and $W_{2}^{\prime}$ are weight functions for $T$. They are finite valued. By Lemma $I .7 .5 \mathrm{~T}$ is $\mathrm{ACW}_{2}$ and $W_{i}$ is an $A C$ part of $W_{2} \cdot$ By Theorem II.I.I3 there is a weight function $W_{\#}$ for $T$ for which $W_{1}^{\prime}+w_{\#}=W_{2}^{\prime}$ a.e. $\mu^{\prime}$ on $\underline{S}^{\prime}$ uniformly with respect to D. By Lemma $I_{07.2, ~} \mathrm{~T}$ is $\mathrm{ACW}_{\#}$. There is a set $\mathrm{M}_{1}$ in $M^{\prime}$ such that $\mu^{\prime} M_{1}^{\prime}=0$ and $w_{1}^{\prime}\left(s^{\prime}, D\right)+w_{\#}^{\prime}\left(s^{\prime}, D\right)=w_{2}^{\prime}\left(s^{\prime}, D\right), s^{\prime} \in C^{\prime} M_{i}^{\prime}$,

$D \in \underline{D}$. Define a non-negative function $w_{0}$ on $\underline{S} \times \underline{D}$ by

$$
W_{0}^{\prime}\left(s^{\prime}, D\right)=\left\{\begin{array}{cl}
W_{\#}\left(s^{\prime}, D\right), & s^{\prime} \in C^{\prime} M_{I}^{\prime}, D \in D \\
0, & s^{\prime} \in M_{I}^{\prime}, D \in \underline{D}
\end{array} .\right.
$$

By Lemma I.7.1 W' is a weight function for T. By Lemma I.7.5 T is $\mathrm{ACW}_{\mathrm{O}} \cdot$ Moreover

$$
w_{0} \leqq w_{2}^{\prime}-w_{i}^{\prime} \leqq w_{S}^{\prime}
$$

Hence $W_{O}^{\prime}$ is an $A C$ part of $W_{S}^{\prime}$ and so $W_{0}^{\prime}$ must be trivial. Hence $W_{\text {\# }}$ is trivial. As a result, $W_{A}^{\prime}=W_{a}^{\prime} a \cdot e \cdot \mu^{\prime}$ on $\underline{S}^{\prime}$ uniformly with respect to $\underline{D}$.

II.2 The general decomposition theorem

In this section we shall prove the "general" decomposition theorem for weight functions. First however, some lemmas are needed. 
II.2.1. Lemma. Let $\left(a_{n i}\right)_{n=1}^{\infty} \infty_{i=1}^{\infty}$ be a double sequence whose entries $a_{n i}$ are real numbers or $+\infty$. Suppose $a_{n i} \leqq a_{n i+1}$, $i \geqq 1, n \geqq 1$, and $a_{n i} \leqq a_{n+1}, i \geqq 1, n \geqq 1$. Then the sequences $\left\{a_{n i}\right\}_{i=1}^{\infty}$ converge (possibly to $+\infty$ ) for $n \geqq 1$ and the sequences $\left\{a_{n i}\right\}_{n=1}^{\infty}$ converge (possibly to $\left.+\infty\right)$ for $i \geqq 1$. Moreover the sequences $\left\{\lim _{i} a_{n i}\right\}_{n=I}^{\infty}$ and $\left\{\lim _{n} a_{n i}\right\}_{i=1}^{\infty}$ converge (possibly to $+\infty)$ and

$$
\lim \operatorname{Iim}_{i} a_{n i}=\operatorname{Iim}_{i} \operatorname{Iim}_{n} a_{n i} \text {. }
$$

The simple proof is omitted.

II.2.2. Lemma. Assume $\left\{W_{11}\right\}_{n=1}^{\infty}$ is a sequence of weight functions for $T$ for which $W_{n}^{\prime} \leqq W_{n+1}^{\prime}, n \geqq I$. (Thus the sequence $\left\{W_{n}^{\prime}\left(s^{\prime}, D\right)\right\}_{n=I}^{\infty}$ is convergent for every $\left(s^{\prime}, D\right)$ in $\left.\underline{S}^{\prime} \underline{D}_{\cdot}\right)$ Define a non-negative function on $\underline{S} \times \underline{D}$ by

$$
w_{0}^{\prime}\left(s^{\prime}, D\right)=\lim w_{n} w^{\prime}\left(s^{\prime}, D\right), \quad\left(s^{\prime}, D\right) \in \underline{s}^{\prime} \times \underline{D} .
$$

Then $W_{0}^{\prime}$ is a weight function for $T$. If $\operatorname{Iim}_{n} W_{n} \underline{S}<\infty$, then $T$ is BVW $_{0}$. If $\lim _{n} W_{n} S<\infty$ and if $T$ is $A C W_{n}(n \geqq 1)$, then $T$ is $A C W_{0}$. Proof. The function $W_{0}^{\prime}$ is indeed non-negative. To show that it is a weight function for $T$ we shall check the four standard conditions of $\mathrm{H} 9$.

i) Suppose $D$ is in $\underline{D}$ and $s^{\prime}$ is in C'TD. Then $w_{o}^{\prime}\left(s^{\prime}, D\right)=\lim _{n} w_{n}^{\prime}\left(s^{\prime}, D\right)=0$. 
ii) Let $\left\{D_{i}\right\}_{i=0}^{m}$ be a finite subset of $\underline{D}$ for which the sets $D_{i}, I \leqq i \leqq m$, are pairwise disjoint and such that $U_{i=1}^{m} D_{i} \subseteq D_{0} \cdot$ Fix $s^{\prime}$ in $S^{\prime} \cdot$ Using the under additivity of each W' we obtain

$$
\begin{aligned}
\sum_{i=1}^{m} W_{0}^{\prime}\left(s^{\prime}, D_{i}\right) & =\sum_{i=1}^{m} \operatorname{Iim} w_{n}^{\prime}\left(s^{\prime}, D_{i}\right) \\
& =\lim \sum_{i=1}^{m} w_{n}^{\prime}\left(s^{\prime}, D_{i}\right) \\
& \leqq \lim { }_{n} w_{n}^{\prime}\left(s^{\prime}, D_{0}\right) \\
& \leqq w_{0}^{\prime}\left(s^{\prime}, D_{0}\right) .
\end{aligned}
$$

Hence W' is under additive.

iii) Suppose a set $D$ in $D$ is the union of a countable number of sets $D_{i}$ in $\underline{D}$ for which $D_{i} \subseteq D_{i+1}, i \geqq 1$. Fix s' in S'. First of all, we have

$$
w_{0}^{\prime}\left(s^{\prime}, D\right)=\lim w_{n} w_{n}^{\prime}\left(s^{\prime}, D\right)=\lim m_{n} \operatorname{Iim}_{i} w_{n}^{\prime}\left(s^{\prime}, D_{i}\right)
$$

The "numbers" $a_{n i}=W_{n}^{\prime}\left(s^{\prime}, D_{i}\right), n \geqq 1, i \geqq 1$, satisfy the requirements of Lemma II.2.1. Thus the sequences $\left\{W_{n}^{\prime}\left(s^{\prime}, D_{i}\right)\right\}_{n=1}^{\infty}$ are convergent and the sequence $\left(\lim _{n} w_{n}^{\prime}\left(s^{1}, D_{i}\right)\right\}_{i=1}^{\infty}$ is convergent; and,

$$
\lim _{n} \operatorname{Iim}_{i} W_{n}^{\prime}\left(s^{\prime}, D_{i}\right)=\operatorname{Iim_{i}} \operatorname{Ii} m_{n} W_{n}^{\prime}\left(s^{\prime}, D_{i}\right)
$$

Combining (1) and (2) yields 


$$
W_{0}^{\prime}\left(s^{\prime}, D\right)=\lim \operatorname{Iim}_{n} W_{n}^{\prime}\left(s^{\prime}, D_{i}\right)=\lim w_{i}\left(s^{\prime}, D_{1}\right)
$$

Hence $W_{O}^{\prime}$ is inner continuous.

$$
\text { iv) Fix } D \text { in } D \text {. Then } W_{j^{\prime}}\left(s^{\prime}, D\right)=\lim w_{n}\left(s^{\prime}, D\right) \text {, }
$$

$s^{\prime} \in \underline{S}^{\prime} \cdot$ Hence the function $W_{0}^{\prime}\left(s^{\prime}, D\right), s^{\prime} \in \underline{S}^{\prime}$, is $\underline{M}^{\prime}$-measurable because each of the functions $w_{n}^{\prime}\left(s^{\prime}, D\right), s^{\prime} \in \underline{S}^{\prime}$, is $\underline{M}^{\prime}-$ measurable.

In view of $i)-i v)$, $W_{0}^{\prime}$ is a weight function for $T$. Now assume also that $\operatorname{Iim}_{n} W_{n} \underline{S}<\infty$. By the Lebesgue Monotone Theorem

$$
\begin{aligned}
w_{0} \underline{S} & =\int_{\underline{\underline{S}}} w_{0^{\prime}}\left(s^{\prime}, \underline{\underline{S}}\right) d \mu^{\prime} \\
& =\lim { }_{n} \int_{\underline{S}} w_{n^{\prime}}\left(\underline{s}^{\prime}, \underline{S}\right) d \mu^{\prime} \\
& =\lim { }_{n} w_{n} \underline{S} .
\end{aligned}
$$

Hence $W_{O} S$ is finite and so $T$ is $B W_{O}$.

Now assume that $\lim _{n} W_{n} S<\infty$ and that $T$ is $A C W_{n}$ for every positive integer $n$. Observe that $T$ is $B V W_{O}$. Since $T$ is $\mathrm{ACW}_{n}$, there is, for each $n$, a non-negative M-measurable function $f_{n}(s)$, $s \in \underline{S}$, such that $W_{n} D=\int_{D} f_{n}(s) d \mu, D \in \underline{D}$. The weight functions $W_{n}^{\prime}$ are monotonically non-decreasing; thus $W_{n} \leqq W_{n+1}$ for every $\mathrm{n} \geqq 1$. Consequently,

$$
\int_{D} f_{n}(s) d \mu \leqq \int_{D} f_{n+1}(s) d \mu, \quad n \geqq 1, D \in \underline{D} .
$$

By Lemma 3.2 of [2] 
(3)

$$
f_{n}(s) \leqq f_{n+1}(s) \text { a.e. } \mu \text { on } \underline{S} \text {, }
$$

for every $n \geqq 1$. Define a non-negative function $f_{0}$ on $\underline{S}$ by

$$
\begin{aligned}
& \lim _{n} f_{n}(s) \text {, if }\left\{f_{n}(s)\right\}_{n=1}^{\infty} \text { is convergent }
\end{aligned}
$$

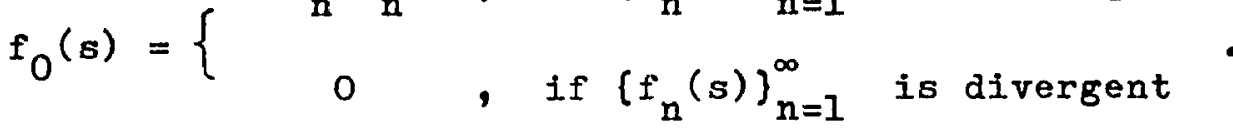

Then $f_{0}$ is non-negative, $\underline{M}$-measurable, and by the Lebesgue

Monotone Theorem we have

$$
\begin{aligned}
w_{0} D & =\int_{\underline{S}^{\prime}} w_{O^{\prime}}\left(s^{\prime}, D\right) d \mu^{\prime} \\
& =\lim \int_{n} \int_{\underline{S}^{\prime}} w_{n^{\prime}}\left(s^{\prime}, D\right) d \mu^{\prime} \\
& =\lim \int_{D} f_{n}\left(s^{\prime} d \mu\right. \\
& =\int_{D} f_{0}(s) d \mu
\end{aligned}
$$

whenever $D$ is in $D$. Hence surely $f_{O}$ is a g.I.b.f. $W_{0}$. Hence $T$ is $\mathrm{ACW}_{0} \cdot$

II.2.3. Lemma. Assume HC3. Let $W_{i}$ and $W_{i}$ be two weight functions for $\mathrm{T}$ for which $\mathrm{T}$ is $\mathrm{ACW}_{1}$ and $\mathrm{ACW}_{2}$. Then the following three conditions are equivalent:

i) $W_{i} \leqq W_{2}^{\prime}$ a.e. $\mu^{\prime}$ on $\underline{S}^{\prime}$ uniformly with respect to $\underline{D}$;

ii) $w_{1}^{\prime}\left(s^{\prime}, D\right) \leqq w_{2}^{\prime}\left(s^{\prime}, D\right)$ a.e. $\mu^{\prime}$ on $\underline{S}^{\prime}$ for every $D$ in $\underline{D}$; iii) $w_{1} \leqq w_{2}$.

Proof. It is clear that $i$ ) implies ii). That ii) implies iii) is equally clear. 
It remains to show that iii) implies i). Thus assume $W_{1} \leqq W_{2}$. Let $f_{i}$ be a g.l.b.f. $W_{i}, i=1,2$. It follows readily from Lemma 3.2 of [2] that $f_{1}(s) \leqq f_{2}(s)$ a.e. $\mu$ on $\underline{S}$.

Suppose $M \prime$ is in $M^{\prime}$ and $D$ is in $D$. As usual, let $H_{M}^{\prime}$ denote the characteristic function of $M^{\prime}$ as a subset of $\underline{S}^{\prime}$. Then, using the transformation formula (Theorem 4.10 of [2]), we obtain

$$
\begin{aligned}
\int_{M^{\prime}} W_{I}^{\prime}\left(s^{\prime}, D\right) d \mu^{\prime} & =\int_{S^{\prime}} H_{M^{\prime}}^{\prime}\left(s^{\prime}\right) W_{1}^{\prime}\left(s^{\prime}, D\right) d \mu^{\prime} \\
& =\int_{D} H_{M^{\prime}}^{\prime} T\left(s^{\prime}\right) f_{I}\left(s^{\prime}\right) d \mu \\
& \leqq \int_{D} H_{M^{\prime}}^{\prime} T\left(s^{\prime}\right) f_{2}\left(s^{\prime}\right) d \mu \\
& \leqq \int_{S^{\prime}} H_{M^{\prime}}^{\prime}\left(s^{\prime}\right) W_{2}^{\prime}\left(s^{\prime}, D\right) d \mu \\
& \leqq \int_{M^{\prime}} W_{2}^{\prime}\left(s^{\prime}, D\right) d \mu^{\prime} \cdot
\end{aligned}
$$

Using the usual measure theory argument, it follows that $W_{i}^{\prime}\left(s^{\prime}, D\right) \leqq W_{2}^{\prime}\left(s^{\prime}, D\right)$ a.e. $\mu^{\prime}$ on $\underline{s}^{\prime} \cdot$ For every $D_{\#}$ in the countable family $\underline{D}_{\#}$ whose existence is guaranteed by HC3, there exists a set $M^{\prime}\left(D_{\# \prime}\right)$ in $\underline{M}^{\prime}$ such that $\mu^{\prime} M^{\prime}\left(D_{\#}\right)=0$ and

$$
W_{1}^{\prime}\left(s^{\prime}, D_{\#}\right) \leqq W_{2}^{\prime}\left(s^{\prime}, D_{\#}\right), \quad s^{\prime} \in C^{\prime} M^{\prime}\left(D_{\#}\right)
$$

Now define $M_{0}^{\prime}=U M^{\prime}\left(D_{\#}\right), D_{\#} \in \underline{D}_{\#} \cdot$ Since $\underline{D}_{\#}$ is countable $M_{0}^{\prime}$ is in $\underline{M}^{\prime}$ and, in fact, $\mu^{\prime} M_{0}^{\prime}=0$. Moreover

$$
w_{1}^{\prime}\left(s^{\prime}, D_{\#}\right) \leqq w_{2}^{\prime}\left(s^{\prime}, D_{\#}\right), s^{\prime} \in C^{\prime} M_{0}^{\prime}, D_{\#} \in \underline{D}_{\#} \cdot
$$


Using HC3 and the inner continuity of the weight functions $W_{i}$ and $W_{2}^{\prime}$, it now follows from (I) that

$$
w_{i}^{\prime}\left(s^{\prime}, D\right) \leqq W_{2}^{\prime}\left(s^{\prime}, D\right), \quad s^{\prime} \in C^{\prime} M_{0}^{\prime}, D \in D .
$$

i) now follows from (2) and the fact that $\mu^{\prime} M_{0}^{\prime}=0$.

Remark. If we assume $\mathrm{HC} 3$, assume that $\mathrm{T}$ is $\mathrm{ACW}_{I}$, assume that $T$ is $A_{C W}$, and assume finally that $W_{1}=W_{2}$, we can deduce that $W_{i}^{\prime}=W_{2}^{\prime}$ a.e. $\mu^{\prime}$ on $\underline{S}^{\prime}$ uniformly with respect to $\underline{D}$. In other words, in this situation, the weights WD, $D \in \underline{D}$, determine the corresponding weight functions essentially uniquely.

II.2.4. Definition. Let $u$ ' be any family of weight functions for $T$. We define a relation on $U$ ' as follows: Suppose $W_{1}^{\prime}$ and $W_{2}^{\prime}$ are in $u^{\prime}$. Then we write $W_{1}<W_{2}^{\prime}$ if and only if $w_{1} \leqq w_{2}$

II.2.5. Remarks. The relation $\preccurlyeq$ is reflexive and transitive on $\mathrm{U}^{\prime}$. Hence $\left(\mathcal{H}^{\prime}, \zeta\right)$ is a partially ordered set. We say that $W_{1}$ precedes $W_{2}$ in the ordering $\leqslant$ if and only if $W_{1}<W_{2}$. $<$ will be termed the standard partial ordering for the family U' of weight functions for $T$.

II.2.6. Lemma. Assume HC3. Assume that $\Omega^{\prime}$ is a countable family of weight functions for $T$. Let $\checkmark$ be the standard partial ordering for $\Omega^{\prime}$. Assume that $\Omega^{\prime}$ is linearly ordered by $\measuredangle$. Assume further that $T$ is $\mathrm{ACW}_{a}$ for every $W_{a}^{\prime}$ in $\Omega \cdot$ 
Then there exists a weight function $W_{0}^{\prime}$ for $T$ for which

1) $W_{0}^{\prime}\left(s^{\prime}, D\right) \leqq \sup \left\{w_{a}^{\prime}\left(s^{\prime}, D\right): W_{a}^{\prime} \in \Omega^{\prime}\right\}$ for every $\left(s^{\prime}, D\right)$ in $\underline{S}^{\prime} \times \underline{D}$;

2) $w_{a} \leqq w_{0}$ for every $w_{a}^{\prime}$ in $\Omega^{\prime}$;

3) if sup $\left\{W_{a} S: W_{a}^{\prime} \in \Omega^{\prime}\right\}<\infty$, then $T$ is $A C W_{0}$

Proof. Given two distinct weight functions $W_{a}^{\prime}$ and $W_{c}^{\prime}$ in $\Omega$ with $W_{a}^{\prime}<W_{c}^{\prime}$, there exists by Lemma II.2.3 a set $M^{\prime}\left(W_{a}^{\prime}, W_{c}^{\prime}\right)$ in $M^{\prime}$ such that $\mu^{\prime} M^{\prime}\left(w_{a}^{\prime}, w_{c}^{\prime}\right)=0$ and such that

$$
w_{a}^{\prime}\left(s^{\prime}, D\right) \leqq w_{c}^{\prime}\left(s^{\prime}, D\right), \quad s^{\prime} \in C^{\prime} M^{\prime}\left(w_{a}^{\prime}, w_{c}^{\prime}\right), D \in \underline{D} .
$$

Define

$$
X^{\prime}=U^{\prime}\left(W_{a}^{\prime}, W_{c}^{\prime}\right), \quad W_{a}^{\prime}, W_{c}^{\prime} \in \Omega^{\prime} \text { and } W_{a}^{\prime}<W_{c}^{\prime}
$$

Then, because $\Omega^{\prime}$ is countable, $X^{\prime}$ is in $M^{\prime}$ and $\mu^{\prime} X^{\prime}=0$. Moreover we have

$$
w_{a}^{\prime}\left(s^{\prime}, D\right) \leqq w_{c}^{\prime}\left(s^{\prime}, D\right), \quad s^{\prime} \in C^{\prime} X^{\prime}, D \in D, w_{a}^{\prime}<w_{c}^{\prime} \in \Omega^{\prime} .
$$

Next, given $w_{a}^{\prime}$ in $\Omega^{\prime}$, we define a non-negative function *W: on $\underline{S} \cdot \underline{D}$ by

$$
* w_{a}^{\prime}\left(s^{\prime}, D\right)=\left\{\begin{array}{cl}
w_{a}^{\prime}\left(s^{\prime}, D\right), & \left(s^{\prime}, D\right) \in C^{\prime} X ' \times D \\
0, & \left(s^{\prime}, D\right) \in X \cdot X D
\end{array} .\right.
$$

Define ${ }^{*} \Omega$ ' to be the family of all non-negative functions $f^{\prime}$ defined on $\underline{S} \times \underline{D}$ for which $f^{\prime}={ }^{*} W_{a}^{\prime}$ for some $w_{a}^{\prime}$ in $\Omega^{\prime}$. In view of (2) and of Lemma I.7.1, * $\Omega$ ' consists of weight functions for T. We can introduce the standard partial ordering <* for the 
family * $\Omega$, of weight functions for T. There is a natural mapping $F$ from $\Omega^{\prime}$ onto $* \Omega$ ' defined by

$$
F\left(w_{a}^{\prime}\right)=* w_{a}^{\prime}, \quad w_{a}^{\prime} \in \Omega \cdot
$$

Because of (2) and because of the fact that $\mu^{\prime} X^{\prime}=0$ we can deduce that $W_{a}^{\prime}=*_{a}^{\prime}$ a.e. $\mu^{\prime}$ on $\underline{S}^{\prime}$ uniformly with respect to D whenever $W_{a}^{\prime}$ is in $\Omega^{\prime}$ and ${ }^{*} W_{a}^{\prime}=F\left(W_{a}^{\prime}\right) ;$ by Lemma $I .7 .5, W_{a}=W_{a}$ and thus $T$ is $A C * W_{a}$ for every ${ }^{*} W_{a}^{\prime}$ in ${ }^{*} \Omega^{\prime}$. Furthermore it follows that $W_{a}^{\prime}<W_{c}^{\prime}$ if and only if $F\left(W_{a}^{\prime}\right)<* F\left(W_{c}^{1}\right)$. Hence $<*$ Iinearly orders * $\Omega$ '. Finally, it follows from (1) and (2) that $W_{a}^{\prime}<* * W_{c}^{\prime}$ if and only if ${ }^{*} W_{a}^{\prime} \leqq W_{c}^{\prime} \cdot$

At last we are ready to introduce the desired function W'́ We define a non-negative function $W_{0}^{\prime}$ on $\underline{S}^{\prime} \times \underline{D}$ by

$$
W_{o}^{\prime}\left(s^{\prime}, D\right)=\sup \left\{w_{a}^{\prime}\left(s^{\prime}, D\right): *_{a}^{\prime} \in w^{*} \Omega^{\prime}\right\},
$$

for every $\left(s^{\prime}, D\right)$ in $\underline{S}^{\prime} \times \underline{D}$. First we shall prove that $W_{0}^{\prime}$ is a weight function for $T$. We must check the four standard requirements of $\mathrm{H9}$.

i) Suppose D is in D and $s^{\prime}$ is in C'TD. The family * $\Omega$ ' consists of weight functions for $T$ so that we must have $w_{0}^{\prime}\left(s^{\prime}, D\right)=\sup \left\{w_{a}^{\prime}\left(s^{\prime}, D\right): w_{a}^{\prime} \in * \Omega^{\prime}\right\}=0$.

ii) Now we assert that wo is under additive. Suppose $D$ in $\underline{D}$ contains a finite number of pairwise disjoint sets $D_{i}$, $I \leqq i \leqq I . ~ F i x s^{\prime}$ in $\underline{S}^{\prime} \cdot$ Two cases must be treated separately. First we assume that there is a positive integer $i_{0}$ for 
which $W_{0}^{\prime}\left(s^{\prime}, D_{i_{0}}\right)=\infty$. In this situation we have $\infty=W_{0}^{\prime}\left(s^{\prime}, D_{i_{0}}\right)=$ $=\sup \left\{{ }^{*} W_{a}^{\prime}\left(s^{\prime}, D_{i_{0}}\right): W_{a}^{\prime} \in R^{\prime}\right\} \leqq \sup \left\{{ }^{*} W_{a}^{\prime}\left(s^{\prime}, D\right):{ }^{*} W_{a}^{\prime} \in *^{*}\right\}=$ $=W_{0}^{\prime}\left(s^{\prime}, D\right) \cdot A s$ a result

(3)

$$
\sum_{i=1}^{I} w_{0}^{\prime}\left(s^{\prime}, D_{i}\right)=\infty=w_{0}^{\prime}\left(s^{\prime}, D\right)
$$

Next, we assume that $w_{0}\left(s^{\prime}, D_{i}\right)<\infty$ for $i=1, \ldots$, I. Fix

$\varepsilon>0$. For each $i=1, \ldots$. , there exists ${ }^{*} W_{i}$ in ${ }^{*} \Omega$ ' such that

$$
{ }^{*} W_{i}\left(s^{\prime}, D_{i}\right)>W_{j}^{\prime}\left(s^{\prime}, D_{i}\right)-\frac{\varepsilon}{I} .
$$

Let ${ }^{*} W_{a}^{\prime}$ be one of the ${ }^{*} W_{i}^{\prime}$ which is preceded by the others in the linear ordering <*. Recall that ${ }^{*} W_{a}^{\prime}$ is under additive and that ${ }^{*} W_{i}<*{ }^{*} W_{a}^{\prime}$ implies ${ }^{*} W_{i} \leqq W_{a}^{\prime}(i=1, \ldots, I)$; we obtain

$$
\begin{aligned}
\sum_{i=1}^{I} w_{0}^{\prime}\left(s^{\prime}, D_{i}\right) & <\sum_{i=I}^{I}\left(w_{i}^{\prime}\left(s^{\prime}, D_{i}\right)+\frac{\varepsilon}{I}\right) \\
& <\sum_{i=I}^{I}\left(w_{a}^{\prime}\left(s^{\prime}, D_{i}\right)+\frac{\varepsilon}{I}\right) \\
& <{ }^{*} w_{a}^{\prime}\left(s^{\prime}, D\right)+\varepsilon \\
& <w_{j}^{\prime}\left(s^{\prime}, D\right)+\varepsilon .
\end{aligned}
$$

Since $\varepsilon>0$ was arbitrary it follows that

$$
\sum_{i=1}^{I} w_{j}^{\prime}\left(s^{\prime}, D_{i}\right) \leqq w_{j}^{\prime}\left(s^{\prime}, D\right) .
$$

It follows from (3) and (4) that $W_{0}^{\prime}$ is under additive.

iii) We assert that $W_{0}^{\prime}$ is inner continuous. Thus 
suppose $D$ in $D$ is the union of a sequence of $\operatorname{sets} D_{i}$, $\geqq 1$, in D for which $D_{i} \subseteq D_{i+1}, i \geqq 1$. Fix $s^{\prime}$ in $\underline{S}^{\prime} \cdot W_{0}^{\prime}$ is under additive and so the sequence $\left\{W_{0}\left(s^{\prime}, D_{i}\right)\right\}_{i=1}^{\infty}$ is convergent and $\lim _{i} W_{0}^{\prime}\left(s^{\prime}, D_{i}\right) \leqq W_{0}^{\prime}\left(s^{\prime}, D\right)$. Suppose $t$ is a real number for which $t<w_{0}^{\prime}\left(s^{\prime}, D\right)$. There exists $w_{a}^{\prime}$ in ${ }^{*} \Omega$, for which $t<{ }^{*} w_{a}^{\prime}\left(s^{\prime}, D\right)$. * W such that $t<w_{a}^{\prime}\left(s^{\prime}, D_{I}\right)$. As a result $t<w_{0}^{\prime}\left(s^{\prime}, D_{I}\right)$. The sequence $\left\{W_{0}^{\prime}\left(s^{\prime}, D_{i}\right)\right\}_{i=I}^{\infty}$ is non-decreasing; we have therefore $t<W_{0}^{\prime}\left(s^{\prime}, D_{I}\right) \leqq \lim _{i} W_{0}^{\prime}\left(s^{\prime}, D_{i}\right)$. It now follows at once that we must have $\lim _{i} W_{0}^{\prime}\left(s^{\prime}, D_{i}\right)=W_{0}^{\prime}\left(s^{\prime}, D\right)$.

iv) Fix $D$ in $\underline{D}$. By definition $w_{0}^{\prime}\left(s^{\prime}, D\right)=\sup \left\{w_{a}^{\prime}\left(s^{\prime}, D\right):^{*} w_{a}^{\prime} \in \Omega^{\prime}\right\}, s^{\prime} \in \underline{s}^{\prime} \cdot$ Because each of the functions ${ }_{a}^{\prime}\left(s^{\prime}, D\right), s^{\prime} \in \underline{S}^{\prime}$, is $\underline{M}^{\prime}-$ measurable and because ${ }^{*} \Omega$ ' is countable, we know that the function $W_{0}^{\prime}\left(s^{\prime}, D\right), s^{\prime} \in \underline{S}^{\prime}$, must be $\underline{M}^{\prime}$-measurable.

Now we wish to show that the weight function $W_{0}^{\prime}$ satisfies conditions 1), 2), and 3) of the conclusion. That it satisfies 1) follows at once from (2). In order to verify 2) we fix $W_{c}$ in $\Omega^{\prime}$ and $D$ in $D$. Then surely

$$
W_{c} D={ }^{*} W_{c} D=\int_{S^{\prime}} *^{\prime} W_{c}\left(s^{\prime}, D\right) d \mu^{\prime} \leqq \int_{S^{\prime}} W_{O}^{\prime}\left(s^{\prime}, D\right) d \mu^{\prime}=W_{O} D \text {. }
$$

Hence 2) holds. 
Condition 3) remains to be checked. We assume that $\sup \left\{W_{a} S: W_{a}^{\prime} \in \Omega^{\prime}\right\}<\infty$. Set $t=\sup \left\{W_{a} L_{a}: W_{a}^{\prime} \in \Omega^{\prime}\right\}$. Surely $t=\sup \left\{{ }^{*} W_{a}:^{*} W_{a}^{\prime} \in *^{*}\right\}$. Select ${ }^{*} W_{1}^{\prime}$ in $* \Omega$ ' so that ${ }^{*} W_{1} \underline{S}>t-1$. Suppose that functions ${ }^{*} w_{1}^{\prime}, \ldots,{ }^{*} w_{m}^{\prime}$ in $* \Omega$ ' have been selected so that

$$
* W_{1} \prec * * W_{2}^{1} \prec * \ldots<* * W_{m}^{\prime}
$$

and

$$
{ }^{*} w_{i} \underline{S}>t-\frac{1}{i}, \quad i=1, \ldots, \text { m. }
$$

Evidently there must exist ${ }^{*} W_{m+1}^{\prime}$ in ${ }^{*} \Omega \prime$ such that

$$
*_{m}^{\prime} \cdot<* W_{m+1}^{\prime}
$$

and

$$
*_{m+I} \underline{S}>t-\frac{I}{m+I}
$$

By induction there exists a sequence $\left\{{ }^{*} W_{m}^{\prime}\right\}_{m=1}^{\infty}$ of weight functions in $* \Omega$ ' such that

$$
* W_{m}^{1}<* * W_{m+1}^{\prime}, m \geqq 1 \text {, }
$$

and

$$
{ }^{*} \mathrm{w}_{\mathrm{m}} \mathrm{S}>t-\frac{\mathrm{I}}{\mathrm{m}}, \mathrm{m} \geqq 1
$$

Combining (5) and an earlier observation yields

$$
{ }^{*} W_{m}^{\prime} \leqq W_{m+I}^{\prime}, \quad m \geqq I \text {. }
$$

The sequence $\left\{W_{m}^{\prime}\left(s^{\prime}, D\right)\right\}_{m=1}^{\infty}$ is non-decreasing and hence is convergent for every $\left(s^{\prime}, D\right)$ in $\underline{S^{\prime}} \times \underline{D}$. Define a non-negative function $W_{\#}$ on $\underline{S}^{\prime} \times \underline{D}$ by 


$$
w_{\#}\left(B^{\prime}, D\right)=\lim *_{m} W_{m}^{\prime}\left(B^{\prime}, D\right), \quad\left(s^{\prime}, D\right) \in S^{\prime} \times D
$$

By Lemma II.2.2 W\# is a weight function for $T$; moreover, since $\operatorname{Iim}_{m} * W_{m} S=t<\infty$ and since $T$ is $A C * W_{m}$ for every $m \geqq I$, it follows that $\mathrm{T}$ is $\mathrm{ACW}_{\#^{-}}$Because of their respective definitions, it is evident that

$$
\text { W } \leqq W_{0} \cdot
$$

In order to strengthen (8) we shall compare W with the functions *W' in ${ }^{\prime} \Omega \prime$. Thus let an arbitrary function $* '$ ' in $* \Omega$ ' be given. Two possibilities arise, because <* linearly orders * $\Omega$ ' Case 1. Assume that there is an integer $m \geqq I$ such that ${ }^{*} W^{\prime}<{ }^{*} W_{m}^{\prime}$ Hence ${ }^{*} W^{\prime} \leqq W_{m}^{\prime}$ and so, using $(7),{ }^{*} W^{\prime} \leqq{ }^{*} W_{m}^{\prime} \leqq W_{\#}^{*}$ Hence

$$
* w^{*} \leqq W_{\#} \cdot
$$

Case 2. Assume that ${ }^{*} W_{m}^{\prime}<* * W^{\prime}$ for every $m \geqq 1$. In this event, we have ${ }^{*} W_{m}^{\prime} \leqq W^{\prime}, m \geqq 1 ; \operatorname{clearly}, W_{\sharp{ }^{\prime}}\left(s^{\prime}, \underline{s}\right) \leqq W^{\prime}\left(s^{\prime}, \underline{s}\right)$,

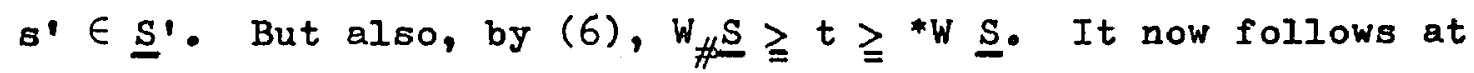
once from these remarks that

$$
W_{\#}\left(s^{\prime}, \underline{S}\right)={ }^{*} W^{\prime}\left(s^{\prime}, \underline{S}\right) \text { a.e. } \mu^{\prime} \text { on } \underline{S}^{\prime} \cdot
$$

According to (9) and (10), we have

$$
*^{\prime}\left(s^{\prime}, \underline{S}\right) \leqq W_{\#}^{\prime}\left(s^{\prime}, \underline{S}\right) \quad \text { a.e. } \mu^{\prime} \text { on } \underline{S}^{\prime}, *^{\prime} \in W^{\prime} \cdot
$$

Inasmuch as * $\Omega$ ' is countable, (II) implies

$$
W_{0}^{\prime}\left(s^{\prime}, \underline{S}\right) \leqq W_{\#}\left(s^{\prime}, \underline{S}\right) \text { a.e. } \mu^{\prime} \text { on } \underline{S}^{\prime} \text {. }
$$

Using (8) and (12), we obtain 
(13)

$$
W_{0}^{\prime}\left(s^{\prime}, \underline{S}\right)=W_{\#}\left(B^{\prime}, \underline{S}\right) \text { a.e. } \mu^{\prime} \text { on } \underline{S}^{\prime} \cdot
$$

Now let $f_{\#}$ be a g.l.b.f. $W_{\#}$ By (13)

$$
W_{0} \underline{S}=\int_{\underline{S}} f_{\#}(s) d \mu \text {. }
$$

But, again by (8),

$$
\int_{D} f_{\#}(s) d \mu=w_{\#} D \leqq w_{0} D, \quad D \in \underline{D} .
$$

From (14) and (15) it follows that $T$ is $\mathrm{ACW}_{0}$.

II.2.7. Theorem. Assume HC3. Let $W_{0}^{\prime}$ and $W_{a}^{\prime}$ be weight functions for $T$. Suppose that $W_{a}^{\prime}$ is an $A C$ part of $W_{j} \cdot$ Suppose that $T$ is $\mathrm{BWW}_{0}$. Then there exists a maximal AC part $W_{A}$ of $W_{0}^{\prime}$ such that $W_{a}^{\prime} \leqq W_{A}^{\prime} \cdot$

Proof. Define $u$ ' to be the family of all weight functions W' for $T$ for which
a) $w_{a}^{\prime} \leqq W^{\prime} \leqq w_{o}^{\prime} ;$
b) $\mathrm{T}$ is ACW.

Let $<$ denote the standard partial ordering of the family $u$ '. Thus $\left(u^{\prime},<\right)$ is a partially ordered set. Observe that the function $W_{a}^{\prime}$ is in $U^{\prime}$. Hence $U^{\prime} \neq \varnothing$.

Now we suppose that $B$ ' is a linearly ordered subfamily of U'. If we can show that has an upper bound in $U^{\prime}$ we may then apply Zorn's Lemma to $\left(u^{\prime}, \prec\right)$. Thus we seek to show that 0 ' does have an upper bound in $U^{\prime}$. The sets of the countable family $\underline{D}_{\#}$ (guaranteed by HC3) can be listed: $\mathrm{D}_{\# 1}, \mathrm{D}_{\# 2}, \ldots, \mathrm{D}_{\# \mathbf{n}}$,

For every positive integer $n$ and every $W^{\prime}$ in $(S)$ have 


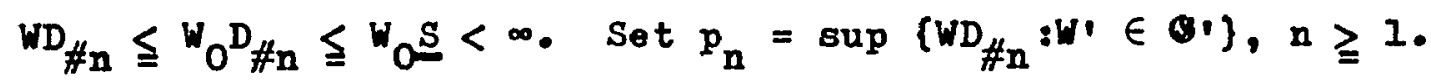

As a result

$$
p_{n} \leqq w_{0} D_{\# n}<\infty, \quad n \geqq 1
$$

Next, for every positive integer $n$, there is by induction a sequence $\left[w_{n m}^{\prime}\right\}_{m=1}^{\infty}$ of functions in $Q^{\prime}$ such that $w_{n m}^{\prime}<w_{n}^{\prime} m+I$, $m \geqq 1$, and such that $W_{n m} D_{\# n}>p_{n}-\frac{I}{m}$. The subfamily $\Omega$ ' of $\mathcal{O}$ ' which consists of all of the $W^{\prime}{ }^{\prime}{ }^{\prime}$ is countable. $\Omega^{\prime}$ is linearly ordered by $<$. Observe also that

$$
\sup \left\{W \underline{S}: W^{\prime} \in \Omega^{\prime}\right\} \leqq W_{O} \leq \infty \text {. }
$$

Hence Lemma II.2.6 applies. There exists a weight function $W_{\#}$ for $T$ for which

$$
\begin{aligned}
& \text { i) } W_{\#}\left(s^{\prime}, D\right) \leqq \sup \left(W^{\prime}\left(s^{\prime}, D\right): W^{\prime} \in \Omega^{\prime}\right\}, \text { for every } \\
& \left(s^{\prime}, D\right) \text { in } \underline{s}^{\prime} \times \underline{D} ; \\
& \text { ii) } W_{\#^{\prime}} W^{\prime} \in \Omega^{\prime} ; \\
& \text { iii) T is ACW } W^{\cdot}
\end{aligned}
$$

Since $\Omega \cdot \subseteq U$ it follows from i) that $W_{\# \leqq W_{0}}$ From ii) it follows that $w_{a} \leqq w_{\#}$ By Lemma II.2.3 $w_{a}^{\prime}\left(s^{\prime}, D\right) \leqq w_{\#}\left(s^{\prime}, D\right)$ a.e. $\mu^{\prime}$ on $\underline{S}^{\prime}$ uniformly with respect to $\underline{D}$. There exists a set $X^{\prime}$ in M' such that

$$
\mu^{\prime} X^{\prime}=0
$$

and

$$
w_{a}^{\prime}\left(s^{\prime}, D\right) \leqq w_{\#}^{\prime}\left(s^{\prime}, D\right), \quad s^{\prime} \in C^{\prime} X^{\prime}, D \in D .
$$


At this point we define a non-negative function $W_{a}^{\prime}$ on $\underline{S}^{\circ} \times \underline{D}$ by

$$
W_{g}^{\prime}\left(s^{\prime}, D\right)=\left\{\begin{array}{ll}
W_{\#}^{\prime}\left(s^{\prime}, D\right), & s^{\prime} \in C^{\prime} X^{\prime}, D \in D \\
W_{a}^{\prime}\left(s^{\prime}, D\right), & s^{\prime} \in X^{\prime}, D \in \underline{D}
\end{array} .\right.
$$

By Lemma I.7.I $W_{\square}^{\prime}$ is a weight function for T. Since $W_{\#} \leqq W_{0}$ and $W_{a}^{\prime} \leqq W_{0}^{\prime}$ it is evident that $W_{b}^{\prime} \leqq W_{0}^{\prime}$ And, using (2), we see that $W_{a}^{\prime} \leqq W_{\square}^{\prime}$ It follows from $(I)$ and Lemma $I .7 .5$ that $W_{\square}=W_{\#}$ and that $T$ is $A C W_{\square}$. Consequently, $W_{\square}^{\prime}$ is in $U^{\prime} \cdot$. From ii), we have

$$
W \leqq W_{\#}=W_{\square}, W^{\prime} \in \Omega^{\prime}
$$

It now follows that $W_{\square} D_{\# n} \geqq W_{n m} D_{\# n}$ for every pair of positive integers $m$ and $n$. Hence

$$
w_{\square} D_{\# n} \geqq p_{n}, \quad n \geqq 1 \text {. }
$$

Now suppose that $W^{\prime}$ is in ${ }^{\prime}$. Then, if we use (4) together with the definition of $p_{n}$, we obtain $W_{\# n} \leqq W_{\square} D_{\# n}, n \geqq l$. If we use $\mathrm{HC} 3$ and the inner continuity of weights in general we obtain

$$
W_{\square} \leqq W^{*}
$$

We therefore proved that $W_{\dot{b}}$ is in $u^{\prime}$ and, in view of (5),

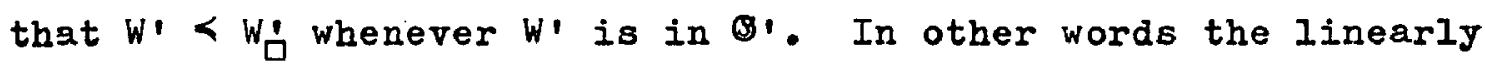
ordered subfamily ${ }^{\prime}$ of $\left(U^{\prime},<\right)$ does admit an upper bound in $U^{\prime}$. By Zorn's Lemma there is an element $W_{A}^{\prime}$ in $u$ ' which is maximal with respect to $\left\langle\right.$; thus, if $W$ in $U^{\prime}$ is comparable with $W_{A}^{\prime}$, 
W' must precede $W_{A}$ in the ordering <. Now, since $W_{A}$ is in $U^{\prime}$, it follows that $W_{a}^{\prime} \leqq W_{A}^{\prime} \leqq W_{O}^{\prime}$ and that $T$ is $A C W_{A}$. Hence $W_{A}^{\prime}$ is an $A C$ part of $W_{0}$ and if we can show that it is a maximal AC part. of $W_{0}^{\prime}$ the proof will be complete.

Thus suppose that ${ }^{W} W^{\prime}$ is an AC part of $W_{O}^{\prime}$ for which $W_{A} \leqq W^{\prime}$ a.e. $\mu^{\prime}$ on $\underline{S}^{\prime}$ uniformly with respect to $D$. There must be a set $M '$ in $M^{\prime}$ such that

$$
H^{\prime} M '=0
$$

and

$$
W_{A}^{\prime}\left(s^{\prime}, D\right) \leqq W^{\prime}\left(s^{\prime}, D\right) \leqq W_{O}^{\prime}\left(s^{\prime}, D\right), \quad s^{\prime} \in C^{\prime} M^{\prime}, D \in \underline{D} .
$$

Define a non-negative function ${ }^{\prime} W^{\prime}$ on $\underline{S}^{\prime} \times \underline{D}$ by

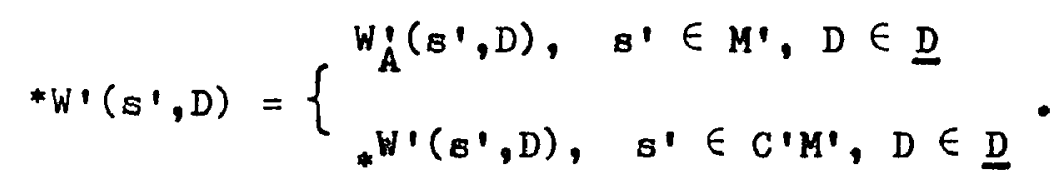

By Lemma I.7.I, *W' is a weight function for T. From (7) and from the fact that $W_{A}^{\prime} \leqq W_{O}^{\prime}$, we deduce that $W_{A}^{\prime} \leqq W^{\prime} \leqq W_{O}^{\prime} \quad$ By

(6) and Lemma I.7.5, we know that

$$
* W=W
$$

and that $T$ is $\mathrm{AC}^{*} W$. Therefore ${ }^{*} W^{\prime}$ is in $U^{\prime} \cdot$ But $W_{A} \leqq{ }^{*} W^{\prime}$ and so $W_{A}<W^{\prime}$. Using the maximality of $W_{A}^{\prime}$, we obtain ${ }^{*} W^{\prime}<W_{A}$ Therefore $* W={ }^{*} W \leqq W_{A}$ (by $\left.(8)\right)$. By Lemma II.2.3, * $W^{\prime} \leqq W_{A}$ a.e. $\mathrm{W}^{\prime}$ on $\underline{S}^{\prime}$ uniformly with respect to $\underline{D}$. In view of the hypothesis concerning $W^{\prime}$, it follows now that $W_{A}^{\prime}={ }^{\prime} W^{\prime} a_{\circ} \theta^{\prime} \mu^{\prime}$ 
on $\underline{S}^{\prime}$ uniformly with respect to $\underline{D}$. Thus W' is a maximal AC part of $W_{0}$.

II.2.8. The General Decomposition Theorem. Assume HC4. Let $W_{0}^{\prime}$ be a weight function for $T$ and suppose that $T$ is $\mathrm{BVW}_{0}$. There exiats a Lebesgue-type decomposition for W'.

Proof. The function $o^{\prime \prime}$ on $\underline{S}^{\prime} \times \underline{D}$ which is defined by $0^{W}\left(s^{\prime}, D\right)=0,\left(s^{\prime}, D\right) \in \underline{S^{\prime}} \times \underline{D}$, is a weight function for $T$. Surely $0^{W '} \leqq W_{0} ;$ and, $T$ is $A C_{0} W$. By Theorem II.2.7 there exists a maximal AC part $W_{a}^{\prime}$ of $W_{0} \cdot$ By Theorem II.1.14 there exist weight functions $W_{A}$ and $W_{S}^{\prime}$ for $T$ such that

$$
\begin{aligned}
& \text { (a) } W_{0}^{\prime}=W_{A}^{\prime}+W_{S}^{\prime} ; \\
& \text { (b) } W_{a}^{\prime}=W_{A}^{\prime} \quad a_{0} \theta \mu^{\prime} \text { on } \underline{S}^{\prime} \text { uniformly with respect to } \underline{D} \text {; } \\
& \text { (c) T is } \mathrm{ACW}_{\mathrm{A}} \text {. }
\end{aligned}
$$

Evidently $W_{A} \leqq W_{0} \cdot$ By Lemma I.7.6, W' is a maximal AC part of $W_{j}$ We are now able to assert that $\left(W_{\dot{A}}, W_{S}^{\prime}\right)$ is a Lebesgue-type decomposition for $W_{j}$. Indeed this follows from (a), (c), the fact that $W_{A}$ is a maximal AC part of $W_{O}$, and Leimma I.7.7.

\section{II.3 A sufficient condition for absolute continuity}

II.3.1. Remarks. Theorem 7.24 of [2] gives a necessary and sufficient condition for $T$ to be ACW; the condition is expressed partly in terms of $T$. The result requires however that 
H13 be satisfied. A similar result for which 113 is not required would be of great interest. The following theorem represents a preliminary step toward such a result.

We continue to assume HI - H8 throughout.

II.3.2. Theorem. Assume H7*, H9, and HCl. Suppose that T is BVW and that $W$ is over additive. Define $S_{+}^{\prime}=$ $=\left\{B^{\prime} \in \underline{S}^{i}: W^{\prime}\left(B^{\prime}, \underline{S}\right)>0\right\}$. Suppose also that whenever B is a set In $\underline{B}$ such that $B \subseteq T^{-1} S_{+}$and $\mu B=0$ it is true that $\mu \cdot T B=0$. Then I is ACW.

Proof. According to 5.11 of [2], it suffices to prove that $W$ is $A C$ with respect to $\mu$.

Note that, since $T$ is BVW, there exists for every $t>0$ a positive real number $\sigma(t)$ such that if $M^{\prime}$ is any set in $M^{\prime}$ with $\mu^{\prime} M^{\prime}<\delta(t)$ then $f_{M^{\prime}} W^{\prime}\left(s^{\prime}, \underline{S}\right) d \mu^{\prime}<t$. Also, according to $H 7^{*}$, whenever $M^{\prime}$ is any element of $\mathcal{M}^{\prime}$ and $\zeta^{\prime}$ is any positive real number there exists a set $O^{\prime}$ in $\mathbb{1} \cdot$ such that $M^{\prime} \subseteq O^{\prime}$ and $\mu^{\prime} O^{\prime} \leqq \mu^{\prime} M^{\prime}+5^{\prime}$

With these general remarks disposed of, we turn to the proof of the assertion that $W$ is AC with respect to $\mu$. Thus suppose $M$ is any set in $\underline{M}$ with $\mu M=0$. Let $\varepsilon>0$ be given. Using Lemma $I .7 .8$ we obtain a set $B$ in $\underline{B}$ such that $M \subseteq B$ and $\mu B=0$. We know by $\mathrm{HCl}$ that TB is in $\underline{M}^{\prime}$. The function $W^{\prime}\left(s^{\prime}, \underline{S}\right)$, $s^{\prime} \in \underline{S}^{\prime}$, is $M^{\prime}-$ measurable and so $S_{+}^{\prime}$ is in $M^{\prime}$. By $H 6$ there must be a set $B_{+}^{\prime}$ in $B^{\prime}$ such that $B_{+}^{\prime} \subseteq S_{+}^{\prime}$ and $\mu^{\prime}\left(S_{+}^{\prime} \cap C^{\prime} B_{+}^{\prime}\right)=0$. 
According to $\mathrm{HCl}, \mathrm{T}^{-1} \mathrm{~B}_{+}$is in $\mathrm{B}$ and $80 \mathrm{~B} \cap \mathrm{T}^{-1} \mathrm{~B}_{+}^{\prime}$ is in $\mathrm{B}$. And, $\mu\left(B \cap T^{-1} B_{+}^{\prime}\right)=0$.

Now we examine the set $T\left(B \cap T^{\left.-I_{B^{\prime}}\right)}\right.$. Evidently $T\left(B \cap T^{\left.-1_{B_{t}}\right)}=\right.$ $=\operatorname{TB} \cap \mathrm{B}_{+}^{\prime}$. Since $B \cap \mathrm{T}^{-1_{B_{+}}} \subseteq \mathrm{T}^{-1} S_{+}$, it follows from the hypothesis that $\mu \cdot\left(\mathrm{TB} \cap \mathrm{B}_{+}^{\prime}\right)=0$. Note that $\mathrm{TB} \cap \mathrm{S}_{+} \subseteq$ $\subseteq\left(T B \cap B_{+}^{\prime}\right) \cup\left(S_{+}^{\prime} \cap C^{\prime} B_{+}^{\prime}\right)$ so that

$$
\mu^{\prime}\left(\mathrm{TB} \cap S_{+}^{\prime}\right) \leqq \mu^{\prime}\left(\mathrm{TB} \cap \mathrm{B}_{+}^{\prime}\right)+\mu^{\prime}\left(S_{+}^{\prime} \cap \mathrm{C}^{\prime} \mathrm{B}_{+}^{\prime}\right) \text {. }
$$

\section{Hence}

$$
H^{\prime}\left(T B \cap S_{+}^{\prime}\right)=0
$$

By $H ?^{*}$ there is a set $O_{0}^{\prime}$ in $\mathbb{D}^{\prime}$ such that $\mathrm{TB} \cap S_{+}^{\prime} \subseteq_{0}^{\prime}$ and $\mu^{\prime} O_{0}<\delta\left(\frac{5}{2}\right)$. There exists a countable number of pairwise disjoint sets $D_{i}, i \geqq 1$, for which $T^{-1} O_{j}=U D_{i}$. Note that $U D_{1}=T^{-1} O_{0} \supseteq T^{-1} T B \cap T^{-1} S_{+} \supseteq B \cap T^{-1} S_{+}$or, in brief,

$$
U D_{i} \supseteq B \cap \mathrm{T}^{-1} S_{+} \cdot
$$

Also $\mathrm{TD}_{i} \subseteq O_{0}^{\prime}$ for $i \geqq 1$ and so

$$
\begin{aligned}
\sum W D_{i} & =\sum \int_{T D_{i}} w^{\prime}\left(s^{\prime}, D_{1}\right) d \mu^{\prime} \\
& =\sum \int_{j^{\prime}} w^{\prime}\left(s^{\prime}, D_{1}\right) d \mu^{\prime} \\
& =\oint_{0} \sum w^{\prime}\left(s^{\prime}, D_{1}\right) d \mu^{\prime} \leq
\end{aligned}
$$




$$
\leqq \oint_{0} w^{\prime}\left(s^{\prime}, \underline{s}\right) d \mu^{\prime}
$$

or

$$
\sum W D_{i}<\frac{\varepsilon}{2}
$$

Next we consider the set $B \cap \mathrm{Cr}^{-1} S_{+}^{\prime}=B \cap \mathrm{T}^{-1} \mathrm{C}^{\prime} \mathrm{S}_{+}$. Note that $T\left(B \cap T^{-1} C^{\prime} S_{+}^{\prime}\right)=T B \cap C^{\prime} S_{+}^{\prime}$. The set $\underline{S}^{\prime}$ can be written as the union of a countable number of sets $B_{i}^{\prime} \perp \geqq 1$, in $\underline{B}^{\prime}$ each of finite $\mu^{\prime}$ measure. Define, for $i \geqq 1, M_{i}^{\prime}=B_{i}^{\prime} \cap T B \cap C^{\prime} S_{+}$Fix $i \geqq 1$. There exists a set $O_{i}^{\prime}$ in $\mathbb{Q D}^{\prime}$ such that $M_{i} \subseteq O_{i}^{\prime}$ and

$$
\mu^{\prime}\left(O_{i}^{\prime} \cap C^{\prime} M_{1}^{\prime}\right)<\delta\left(\frac{\varepsilon}{2^{i+1}}\right)
$$

There is a countable number of pairwise disjoint sets $D_{i j}, j \geqq 1$, in $D$ such that $U_{j} D_{i j}=T^{-1} 0_{i}$. Note that $U_{i, j} D_{1 j}=U_{i} T^{-1} 0_{i} \supseteqq$ $\supseteq U_{i} T^{-1} M_{i}=T^{-1} T B \cap T^{-1} C^{i} S_{+}$, so that we have

$$
U_{i, j} D_{i j} \stackrel{\supseteq}{=} B \cap C T^{-1} S_{+} \cdot
$$

The positive integer i is again fixed. We have

$$
O_{i} \cap C^{\prime M} !=\left(O_{i} \cap C^{\prime} B_{i}^{\prime}\right) \cup\left(O_{i} \cap C \cdot T B\right) \cup\left(O_{i}^{\prime} \cap s_{+}^{\prime}\right)
$$

so that $O_{i} \cap C^{\prime} M_{i} \supseteq O_{i} \cap s_{+}$. Hence $\mu \cdot\left(O_{i} \cap s_{+}^{\prime}\right)<\sigma\left(\frac{\varepsilon}{2^{i+1}}\right)$. We have $\mathrm{TD}_{i j} \subseteq O_{i}$ for $i \geqq 1$ and $j \geqq 1$. Hénce

$$
\sum_{i, j} W D_{i j}=\sum_{i} \sum_{j} \int_{T D_{i j}} w^{\prime}\left(s^{\prime}, D_{i j}\right) d \mu^{\prime}=
$$




$$
\begin{aligned}
& =\sum_{i} \sum_{j} \int_{D_{i}} w^{\prime}\left(s^{\prime}, D_{i j}\right) d \mu^{\prime} \\
& \leqq \sum_{i} \int_{i} w^{\prime}\left(s^{\prime}, s\right) d \mu \cdot \\
& \leqq \sum_{1} \int_{D^{\prime} n s_{+}} w^{\prime}\left(s^{\prime}, \underline{s}\right) d \mu^{\prime} \\
& <\sum_{i \geq 1} \frac{e}{2^{i+1}}
\end{aligned}
$$

Thus we have

$$
\sum_{i, j} W D_{i j}<\frac{\varepsilon}{2} .
$$

In view of (2) and (4), we have $M \subseteq B=\left(B \cap T^{-1} S_{t}^{1}\right) U$

$U\left(B \cap C T^{-1} S_{+}^{\prime}\right) \subseteq U_{i} D_{1} U U_{i, j} D_{i j}$ or

$$
M \subseteq U_{i} D_{i} U U_{i, j} D_{i j}
$$

And, by (3) and (5),

(7)

$$
\sum_{i} W D_{i}+\sum_{i, j} W D_{i j}<\varepsilon_{.}
$$

It follows from (6) and (7) that $W$ is $A C$ with respect to $\mu$. Hence $T$ is ACW.

II.4 On the difference of two almost over additive weight functions

We shall not examine the difference of two arbitrary almost over additive weight functions. Such a difference may not be 
non-negative and so it could hardy be a weight function. We shall however examine the same special case as that considered in Section II.1. We shall suppose that $W^{\prime}$ and $W_{A}$ are weight functions for $T$ and that $W_{A}^{\prime}$ is a finite valued $A C$ part of $W^{\prime}$. It will then be proved that, under certain conditions, $W^{\prime}-W_{A}$ is almost over additive if $W^{\prime}$ is almost over additive. We Bhall continue to assume Hl - H8.

\section{II.4.1. Lemma. Assume H4* and H9. Suppose $M$ is in $M$} with $\mu M<\infty$ and suppose that $D_{0}$ is a set in $D$ with $M \subseteq D_{0}$. Then there is a double sequence $\left\{D_{k i}\right\}_{k=1}^{\infty} \prod_{i=1}^{\infty}$ of sets in $\underline{D}$ such that

$$
\begin{aligned}
& \text { (1) } \quad M \subseteq U_{i \geq I} D_{k i} \subseteq U_{i \geq 1} D_{k-1} i^{\prime} \\
& \text { (ii) } \Sigma_{i \geq 1} W^{\prime}\left(s^{\prime}, D_{k i}\right) \leqq \Sigma_{i \geq 1} W^{\prime}\left(s^{\prime}, D_{k-1}\right)^{\prime}, s^{\prime} \in \underline{s}^{\prime}, \\
& \text { for every } k>1 \text { and such that } \\
& \text { (iii) the sets } D_{k i}(i \geqq l) \text { are pairwise disjoint, } \\
& \text { (iv) } \mu\left(U_{i \geqq 1} D_{k i} \cap \mathrm{CM}\right)<\frac{1}{\mathbf{k}} \\
& \text { (v) } U_{1 \geqq I} D_{k i} \cong D_{0} \cdot
\end{aligned}
$$

Proof. By H4* there is, for every positive integer $k$, a countable number of pairwise disjoint sets $D_{k i}^{* *}, i \geqq 1$, in $\underline{D}$ such that $M \subseteq U_{i \geqq l} D_{k i}^{* *}$ and $\Sigma_{i \geqq l} \mu D_{k i}^{* *}<\mu M+\frac{1}{k}$. For every positive Integer 1 , there is a countable number of pairwise disjoint sets

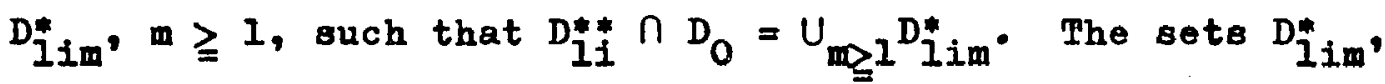


$1 \geqq 1, m \geqq 1$, are pairwise disjoint subsets of $D_{0}$ in $\underline{D}$ for which

$$
M \subseteq D_{0} \cap U_{i \geq I^{\prime}} D_{i i}^{* *}=U_{i, m} D_{i \text { im }}^{*}
$$

and

$$
\sum_{i, m} \mu D_{i \text { im }}^{*} \leqq \sum_{i \geqslant I} \mu D_{l i}^{* *}<\mu M+\frac{1}{1}
$$

Hence there exist a countable number of sets $D_{1 i}, i \geqq 1$, in $\underline{D}$

for which (iii), (iv), and ( $v$ ) hold when $I \leqq k \leqq I$ and

$M \subseteq U_{i} D_{1 i}$

Assume now that for all positive integers $k$ which do not exceed p sets $D_{k i}, i \geqq 1$, in $\underline{D}$ have been chosen so that ( $i$ ) and (ii) hold for $2 \leqq k \leqq p$ and (iii), (iv), and ( $v$ ) hold for

$I \leqq k \leqq p$. For every pair of positive integers $i$ and $m$ there is a countable number of pairwise disjoint sets $D_{i m u}^{(p)}, u \geqq 1$, in $\underline{D}$ such that

$$
D_{p+1}^{* *} i \cap D_{p m}=U_{u \geq 1} D_{i m u}^{(p)}
$$

Note that the sets $p_{i m u}^{(p)}, i \geqq 1, m \geqq 1, u \geqq 1$, are pairwise disjoint sets in $\underline{D}$. Now also

$$
U_{i, m, u} D_{i m u}^{(p)}=U_{i \geq 1} D_{p+1}^{* *} \cap U_{m \notin 1} D_{p m} \cdot
$$

Hence we have

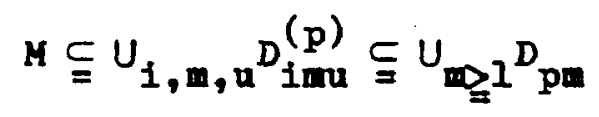

and 


$$
\mu\left(U_{i, m, u} D_{i m u}^{(p)} \cap C M\right) \leqq \mu\left(U_{i} D_{p+1}^{* *} i \cap C M\right)<\frac{1}{p+1}
$$

In addition

$$
D_{\text {imu }}^{(p)} \cong D_{p m} \cong D_{0}, i \geqq 1, m \geqq 1, u \geqq 1 \text {. }
$$

Inasmuch as $W^{\prime}$ is under additive and since, for each $m \geqq I$, the sets $D_{i m u}^{(p)}, i \geqq I, u \geqq I$, are pairwise disjoint subsets of $D_{p m}$ it follows that

$$
\sum_{i, m, u} w^{\prime}\left(s^{\prime}, D_{i m u}^{(p)}\right) \leqq \sum_{m \geq I} w^{\prime}\left(s^{\prime}, D_{p m}\right), s^{\prime} \in S^{\prime} \cdot
$$

We list the sets $D_{i m u}^{(p)}, i \geqq I, m \geqq I, u \geqq I$, as:

$$
D_{p+1}, D_{p+1}, \cdots, D_{p+1}, \cdots
$$

It is evident from these remarks and from the induction hypothesis that the sets $D_{k i}, 1 \leqq k \leqq p+1, i \geqq 1$, satisfy (i) and (ii) for $2 \leqq k \leqq p+1$ and (iii), (iv), and (v) for $1 \leqq k \leqq p+1$

The proof is completed by an inductive argument.

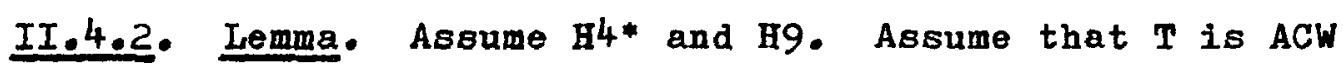
and let $f$ be a B.l.b.f. W. Suppose $M$ is a set in $M$ with $\mu M<\infty$. Let $\left\{D_{k i}\right\}_{k=1}^{\infty} \sum_{i=1}^{\infty}$ be a double sequence of sets in $D$ for which conditions (i) - (iv) of Lemma II.4.I hold. Then the sequence $\left\{\Sigma_{i \geqq 1} W^{\prime}\left(s^{\prime}, D_{k i}\right)\right\}_{k=1}^{\infty}$ is convergent for every $s^{\prime}$ in $\underline{S}^{\prime}$ and if we define a non-negative function $g^{\prime}$ on $\underline{S}^{\prime}$ by $g^{\prime}\left(s^{\prime}\right)=$ 
$=\lim _{k} \Sigma_{i \geq 1} W^{\prime}\left(s^{\prime}, D_{k i}\right), s^{\prime} \in \underline{S}^{\prime}$, then $g^{\prime}\left(s^{\prime}\right), s^{\prime} \in \underline{S}^{\prime}$, is $\underline{H}^{\prime}-$

measurable. Moreover

$$
\int_{S^{\prime}} B^{\prime}\left(B^{\prime}\right) d \mu^{\prime}=\int_{M} f(s) d \mu .
$$

Proof. The convergence of each sequence $\left\{\Sigma_{i \geq I^{1}} W^{\prime}\left(s^{\prime}, D_{k i}\right)\right\}_{k=1}^{\infty}$

follows from (ii). The function $g^{\prime}\left(s^{\prime}\right)$, $s^{\prime} \in \underline{S}^{\prime}$, is $\underline{M}^{\prime}-$

measurable because it is the limit of a sequence of $\underline{M}^{\prime}$-measurable functions. The functions in the non-increasing sequence

$\left\{\Sigma_{i \geqq I} W^{\prime}\left(s^{\prime}, D_{k i}\right), s^{\prime} \in \underline{s}^{\prime}\right\}_{k=1}^{\infty}$ are bounded by the $\mu^{\prime}$-integrable function $W^{\prime}\left(s^{\prime}, \underline{S}\right)$, s' $\in \underline{S}^{\prime}$. Hence it follows from the Lebesgue Theorem that

$$
\int_{\underline{S}} G^{\prime}\left(s^{\prime}\right) d \mu^{\prime}=1 i \xi_{k} \int_{\underline{S}} \sum_{i \geqq 1} W^{\prime}\left(s^{\prime}, D_{k i}\right) d \mu^{\prime} \cdot
$$

Now $f$ is a g.l.b.f. W so that we have

$$
\begin{gathered}
\int_{\underline{\underline{S}}} \sum_{i \geqq I} w^{\prime}\left(s^{\prime}, D_{k i}\right) d \mu^{\prime}=\sum_{i \geqq I} \int_{\underline{S^{\prime}}} w^{\prime}\left(s^{\prime}, D_{k i}\right) d \mu^{\prime}= \\
=\sum_{i \geqq I} \int_{D_{k i}} f(s) d \mu=\int_{U_{i} D_{k i}} f(s) d \mu
\end{gathered}
$$

for every positive integer $k$. Using (i) we obtain

$$
\int_{\underline{S}} \sum_{i \geqq I} W^{\prime}\left(s^{\prime}, D_{k i}\right) d \mu^{\prime}=\int_{M} f(s) d \mu+\oint_{i} D_{k i} \cap C M^{f}(s) d \mu \cdot
$$

Set $N_{k}=U_{i} D_{k i} \cap C M$ and $N=n_{k} U_{i} D_{k i} \cap C M$. In view of (i) the 
sequence $\left(\int_{k_{k}} f(s) d \psi\right\}_{k=1}^{\infty}$ is non-increasing and hence convergent; indeed, since the integrals $\delta_{\mathrm{N}_{k}} f(s) d \mu, k \geqq 1$, are finite, we have $\lim _{k} \int_{N_{k}} f(s) d \mu=\delta_{N} f(s) d \mu$. But, by (iv) $\mu N=0$. Hence

$$
\operatorname{Iim}_{k} \int_{i} D_{k i} \cap C M(s) d \mu=0 .
$$

Recall that (2) is valid for every $k \geqq 1$. As a result

$$
\begin{aligned}
\lim m_{k} & \int_{\underline{S}} \sum_{i \geq I} w^{\prime}\left(s^{\prime}, D_{k i}\right) d \mu^{\prime}= \\
& =\int_{M} f(s) d \mu+\lim _{k} \int_{D_{i} D_{k i} \text { nCM }} f(s) d \mu .
\end{aligned}
$$

It follows from (1), (4), and (3) that

$$
\int_{\underline{S}} B^{\prime}\left(B^{\prime}\right) d \mu^{\prime}=\int_{M} f(s) d \mu .
$$

II.4.3. Lemma. Assume H4*. Suppose that $W^{\prime}$ and $W_{A}^{\prime}$ are weight functions for $T$. Assume that $W_{A}$ is a finfte valued $A C$ part of $W^{\prime}$. Assume also that $W^{\prime}$ is almost orer additive. Define a non-negative function $W_{\dot{A}}^{\prime}$ on $\underline{S}^{\prime} \times \underline{D}$ by

$$
w_{\Delta}^{\prime}\left(s^{\prime}, D\right)=W^{\prime}\left(s^{\prime}, D\right)-w_{A}^{\prime}\left(s^{\prime}, D\right), \quad\left(s^{\prime}, D\right) \in \underline{S}^{\prime} \times \underline{D} .
$$

Let $\left(D_{k}\right\}_{k=0}^{n}$ be a finite sequence of sets in $\underline{D}$ for which $D_{0} \subseteq U_{k=1}^{n} D_{k}$. Assume also that $\mu D_{0}$ is finite. Then

$$
w_{\Delta}^{\prime}\left(s^{\prime}, D_{0}\right) \leqq \sum_{k=1}^{n} w_{\Delta}\left(s^{\prime}, D_{k}\right) \text { a.ee. } \omega^{\prime} \text { on } \underline{S}^{\prime} \text {. }
$$




\section{Proof. By Lemma II.1.9 the function $W_{\Delta}$ is almost under}

additive. If $n=1$ the conclusion follows from the almost under additivity of $W_{\Delta} \cdot$ Assume then that $n \geqq 2$.

Define $E_{1}=D_{0} \cap D_{1}$ and, for $k=2, \ldots, n$, define

$E_{k}=D_{0} \cap D_{k} \cap n_{i=1}^{k-1} C D_{i}$. Each $E_{k}$ is in $\underline{M}$ and $\mu E_{k} \leqq \mu D_{0}<\infty$.

The sets $E_{k}, k=1, \ldots, n$, are pairwise disjoint and

$$
D_{0}=U_{k=1}^{n} E_{k}
$$

Observe also that $E_{k} \subseteq D_{k}, I \leqq k \leqq n$. By Lemma II.4.I there exists, for $k=1, \ldots, n$, a double sequence of sets $D_{k p i}$,

$p \geqq 1, i \geqq 1$, in $\underline{D}$ such that

$$
\begin{aligned}
& \text { (i) } \quad E_{k} \subseteq U_{i \gg I} D_{k p i} \subseteq U_{i \gg I} D_{k} p-I i^{\prime} \\
& \text { (ii) } \Sigma_{i \geqq l} W_{A}^{\prime}\left(s^{\prime}, D_{k p i}\right) \leqq \Sigma_{i \geqq l} W_{A^{\prime}}^{\prime} s^{\prime}, D_{k}^{\prime} p-1 i^{\prime}, s^{\prime} \in \underline{S}^{\prime},
\end{aligned}
$$

for every $p>1$ and such that it is also true that

(iii) the sets $D_{k p I}(i \geqq 1$ ) are pairwise disjoint,

$$
\begin{aligned}
& \text { (iv) } \mu\left(U_{i \geq I} D_{k p i} \cap C E_{k}\right)<\frac{I}{p}, \\
& \text { (v) } D_{k p i} \subseteq D_{k}, i \geqq 1
\end{aligned}
$$

for every $p \geqq 1$. Let $f_{A}$ be a $8.1 . b . f . W_{A}$. By Lemma II.4.2 the sequence $\left\{\Sigma_{i \geqq l} W_{A}\left(s^{\prime}, D_{k p 1}\right)\right\}_{p=1}^{\infty}$ is convergent for every $s^{\prime}$ in $\underline{S}^{\prime}$ and if 


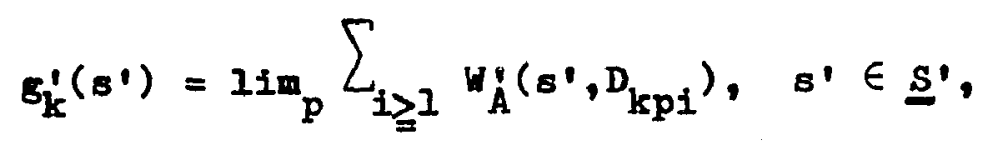

then $B_{k}^{\prime}\left(s^{\prime}\right), s^{\prime} \in \underline{S}^{\prime}$, is $\underline{M}^{\prime}-$ measurable; and,

$$
\int_{S^{\prime}} \delta_{k}^{\prime}\left(B^{\prime}\right) d \mu^{\prime}=\int_{\mathbb{F}_{k}} f_{A}(B) d \mu, \quad 1 \leqq k \leqq n \text {. }
$$

At this point we fix the positive integer p. According to (iii) and (v), the sets $D_{k p i}, i \geqq 1$, are pairwise disjoint subsets of $D_{k}$ for each $k$. $W_{\dot{\Delta}}$ is almost under additive; hence

$$
\sum_{i \geq 1} w_{\Delta}\left(s^{\prime}, D_{k p i}\right) \leqq w_{\Delta}\left(s^{\prime}, D_{k}\right) \text { a.e. } \mu^{\prime} \text { on } \underline{s}^{\prime}, 1 \leqq k \leqq n \text {. }
$$

In view of ( 1 ) and ( $i$ ) we have

$$
D_{0} \cong U_{k=1}^{n} U_{i \geq 1} D_{k p i} \text {. }
$$

The function $W$ ' is almost over additive. It follows that

$$
W^{\prime}\left(s^{\prime}, D_{0}\right) \leqq \sum_{k=1}^{n} \sum_{i \geq 1} w^{\prime}\left(s^{\prime}, D_{k p i}\right) \text { a.e. } \mu^{\prime} \text { on } \underline{S}^{\prime} \text {. }
$$

Consequently

$$
\begin{aligned}
& w_{\dot{\Delta}}\left(s^{\prime}, D_{0}\right) \leqq \sum_{k=1}^{n} \sum_{i \geq 1} w^{\prime}\left(s^{\prime}, D_{k p i}\right)-w_{A}\left(s^{\prime}, D_{0}\right) \\
& \text { a.e. } \mu^{\prime} \text { on } \underline{s}^{\prime} \text {. }
\end{aligned}
$$

The function $\Sigma_{k=1}^{n} \Sigma_{i \geqq 1} W_{A}^{\prime}\left(s^{\prime}, P_{k p i}\right), s^{\prime} \in \underline{S}^{\prime}$, is real valued for indeed 


$$
\sum_{k=1}^{n} \sum_{i \gg 1} w_{A}^{\prime}\left(s^{\prime}, D_{k p i}\right) \leqq n \cdot w_{A}\left(s^{\prime}, \underline{S}\right)<\infty .
$$

From (5) we obtain

$$
\begin{aligned}
& w_{\Delta}^{\prime}\left(s^{\prime}, D_{0}\right) \leqq \sum_{k=1}^{n} \sum_{i \geq 1} w^{\prime}\left(s^{\prime}, D_{k p i}\right)-\sum_{k=1}^{n} \sum_{i \geq I^{\prime}} w_{i}\left(s^{\prime}, D_{k p 1}\right)+ \\
& +\left\{\sum_{k=1}^{n} \sum_{i \geq 1} w_{A}^{\prime}\left(s^{\prime}, D_{k p i}\right)-w_{A}^{\prime}\left(s^{\prime}, D_{0}\right)\right\} \text { a,e, } \mu^{\prime} \text { on } \underline{s}^{\prime}
\end{aligned}
$$

and so

$$
\begin{aligned}
& w_{\Delta}^{\prime}\left(s^{\prime}, D_{0}\right) \leqq \sum_{k=1}^{n} \sum_{i \geq 1} w_{\Delta}^{\prime}\left(s^{\prime}, D_{k p i}\right)+ \\
& +\left\{\sum_{k=1}^{n} \sum_{i \geq 1} w_{A}^{\prime}\left(s^{\prime}, D_{k p i}\right)-w_{A}^{\prime}\left(s^{\prime}, D_{0}\right)\right\} \text { a.e. } \mu^{\prime} \text { on } \underline{s}^{\prime} \cdot
\end{aligned}
$$

Combining (3) and (6) yields

$$
\begin{aligned}
& w_{\Delta}\left(s^{\prime}, D_{0}\right) \leqq \sum_{k=1}^{n} w_{\Delta}\left(s^{\prime}, D_{k}\right)+ \\
& +\left\{\sum_{k=1}^{n} \sum_{i \geqq 1} w_{A}^{\prime}\left(s^{\prime}, D_{k p i}\right)-w_{A}^{\prime}\left(s^{\prime}, D_{0}\right)\right\} \text { a.e. } \mu^{\prime} \text { on } \underline{s}^{\prime} \cdot
\end{aligned}
$$

Expression (7) is valid for every positive integer p.

It follows now from (7) that

$$
\begin{aligned}
& w_{\Delta}\left(s^{\prime}, D_{0}\right) \leqq \sum_{k=1}^{n} w_{\Delta}^{\prime}\left(s^{\prime}, D_{k}\right)+\sum_{k=1}^{n} B_{k}^{\prime}\left(s^{\prime}\right)-w_{A}\left(s^{\prime}, D_{0}\right) \\
& \text { a.e. } \mu^{\prime} \text { on } \underline{s}^{\prime} \cdot
\end{aligned}
$$

By 6.1 of [2] the function $W_{A}^{\prime}$ is almost over additive. Thus, in 


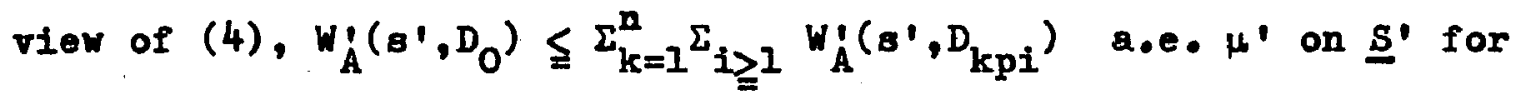
every positive integer p. Hence, referring again to the definition of $g_{k}^{\prime}\left(s^{\prime}\right), s^{\prime} \in \underline{S}^{\prime}$, we obtain

$$
W_{A}\left(s^{\prime}, D_{0}\right) \leqq \sum_{k=1}^{n} g_{k}^{\prime}\left(s^{\prime}\right) \text { a.e. } \mu^{\prime} \text { on } \underline{S}^{\prime} \cdot
$$

Using (9), (2), and (1) successively, we note that

$$
\begin{aligned}
& \int_{D_{0}} f_{A}(s) d \mu=\int_{S^{\prime}} W_{A^{\prime}}\left(s^{\prime}, D_{0}\right) d \mu^{\prime} \leqq \int_{\underline{S}} \sum_{k=1}^{n} g_{k}^{\prime}\left(s^{\prime}\right) d \mu^{\prime}= \\
& =\sum_{k=1}^{n} \int_{S^{\prime}} g_{k}^{\prime}\left(s^{\prime}\right) d \mu^{\prime}=\sum_{k=1}^{n} \int_{B_{k}} f_{A}(s) d \mu= \\
& =\int_{D_{0}} f_{A}(s) d \mu<\infty .
\end{aligned}
$$

Hence

$$
\int_{\underline{S}}, w_{A}\left(s^{\prime}, D_{0}\right) d \mu^{\prime}=\int_{\underline{S}} \sum_{k=I}^{n} B_{k}^{\prime}\left(s^{\prime}\right) d \mu^{\prime}<\infty .
$$

In view of (9) and (10) it follows at once that

$$
W_{A}\left(s^{\prime}, D_{0}\right)=\sum_{k=1}^{n} g_{k}^{\prime}\left(s^{\prime}\right) \text { a.e. } \mu^{\prime} \text { on } \underline{S}^{\prime} \cdot
$$

Because of (8) and (11) we have finally

$$
W_{\Delta}^{\prime}\left(s^{\prime}, D_{0}\right) \leqq \sum_{k=1}^{n} w_{\Delta}^{\prime}\left(s^{\prime}, D_{k}\right) \text { a.e. } \mu^{\prime} \text { on } \underline{S}^{\prime} \cdot
$$

II.4.4. Lemma. Assume H4* and HC2. Suppose W' and $W_{A}$ are weight functions for $T$. Suppose that $W_{A}^{\prime}$ is a finite valued 
AC part of W'. Assume moreover that W' is almost over additive. Define a non-negative function $w_{\Delta}^{\prime}$ on $\underline{S}^{\prime} \times \underline{D}$ by $w_{\Delta}^{\prime}\left(B^{\prime}, D\right)=$ $W^{\prime}\left(s^{\prime}, D\right)-W_{A}^{\prime}\left(s^{\prime}, D\right),\left(s^{\prime}, D\right) \in \underline{S}^{\prime} X \underline{D}$. Then $W_{\Delta}^{\prime}$ is almost over additive.

Proof. Let $D^{j}, j \geqq 0$, be a countable family of sets in $\underline{J}$ for which

$$
D^{0} \subseteq U_{j \geqq 1} D^{j}
$$

By HC2 there is a sequence of sets $D_{i}, i \geqq l$, in $D$ for which the following properties hold:

(i) $D_{i} \subseteq D_{i+1}, \quad i \geqq 1$;

(ii) $D^{0}=U_{i \geqq 1} D_{i}$;

(iii) if ${ }^{\prime} D^{j}, j \geqq l$, is any countable family of sets in D for which $D^{0} \subseteq U_{j \geqq 1} D^{j}$ then for every positive integer $i$ there exists a positive integer $J(i)$ such that $D_{1} \subseteq U_{j \geqq 1}^{J(i)} \#_{D}^{j}$.

By 14 there exists a countable family of sets ${ } D_{j}, j \geqq I$, in $D$ such that $\underline{S}=U_{j \geqq I} D_{j}$ and $\mu_{*} D_{j}$ is finite for $j \geqq 1$. By (iii) there is for every positive integer $i$ a positive integer $J^{*}(i)$ such that $D_{i} \subseteq U_{j=1}^{J *(i)}{ }_{*} D_{j}$. Thus $\mu D_{i} \leqq \Sigma_{j=1}^{J *(i)} \mu_{*} D_{j}, i \geqq 1$. In other words we have 
(2)

$$
\mu D_{i}<\infty, \quad i \geqq 1
$$

Furthermore, according to (iii) and (1), there exists for every positive integer $i$ positive integer $j(i)$ such that

$$
D_{1} \subseteq U_{j=1}^{j(1)} D^{j}, \cdot 1 \geqq 1 \text {. }
$$

Fix a positive integer I. From (2) and (3) we deduce $\mu D_{I}<\infty$ and $D_{I} \subseteq U_{j=I}^{j(I)} D^{j}$. We apply Lemma II.4.3 to obtain $W_{\Delta}\left(B^{\prime}, D_{I}\right) \leqq \Sigma_{j=I}^{j(I)} W_{\Delta}\left(s^{\prime}, D^{j}\right)$ a.e. $H^{\prime}$ on $\underline{S}^{\prime} \cdot$ Hence surely

$$
w_{\Delta}\left(B^{\prime}, D_{I}\right) \leqq \sum_{j \geqq I} w_{\Delta^{\prime}}\left(s^{\prime}, D^{j}\right) \quad \text { a.e. } \mu^{\prime} \text { on } \underline{S}^{\prime} \cdot
$$

Expression (4) is valid for every positive integer I.

It was proved in Lemma II.1.9 that $W_{\Delta}^{\prime}$ is inner continuous. Using this fact together with (i), (ii), and (4), we obtain $W_{\Delta}^{\prime}\left(s^{\prime}, D^{0}\right) \leqq \Sigma \Sigma_{j \geqq 1} W_{\Delta}^{\prime}\left(s^{\prime}, D^{j}\right)$ a.e. $\mu^{\prime}$ on $\underline{S}^{\prime}$. Hence $W_{\Delta}^{\prime}$ is almost over additive.

II.5 The Strong Decomposition Theorem

First we prove a lemma about almost over additive weight functions which are singular. Then we proceed to the decomposition results.

II.5.1. Lemma. Assume H9, H7*, and HCl. Assume that W' is almost over additive and that $T$ is BW. Suppose also that W' is singular. Then $W^{\prime}$ is strongly singular. Proof. Since the measure space $\left(\underline{S}^{\prime}, \underline{M}^{\prime}, \mu^{\prime}\right)$ is $\sigma$-finite 
there is a countable number of pairwise disjoint setB $M_{n}^{\prime}, n \geqq 1$, in $\underline{y}^{\prime}$ for which $\underline{S}^{\prime}=U_{n \geq I} M_{n}^{\prime}$ and $\mu^{\prime} M_{n}^{\prime}<\infty, n \geqq 1$. Define

$$
S_{+}^{\prime}=\left\{s^{\prime} \in \underline{S}^{\prime}: W^{\prime}\left(s^{\prime}, \underline{S}\right)>0\right\} \text {. }
$$

Define $\Gamma$ to be the family of all sets $B$ in $\underline{B}$ for which $\mu B=0$. The union of a countable number of sets in $\Gamma$ is again in $\Gamma$. Also, for every positive integer $n$, define $\Gamma_{n}^{\prime}$ to be the family of all sets $M^{\prime}$ in $M^{\prime}$ for which $M^{\prime}=M_{\mathfrak{n}}^{\prime} \cap T$ for some $B$ in $\Gamma$. Set $p_{n}=\sup \left\{\mu^{\prime} M^{\prime}: M \prime \in \Gamma_{n}^{\prime}\right\}, n \geqq 1$. Note that $p_{n} \leqq \mu^{\prime} M_{n}^{\prime}<\infty, n \geqq 1$. Now fix an arbitrary positive integer $n$. There exists a sequence of sets $B_{n m}$, $m \geqq 1$, in $\Gamma$ such that

$$
\lim _{m} \mu \cdot\left(M_{n}^{\prime} \cap \mathbb{T B}_{n m}\right)=p_{n} \text {. }
$$

Introduce $B_{n}=U_{m \geq 1} B_{n m}$. Since $\Gamma$ is closed under countable unions it follows that $B_{n}$ is in $\Gamma$. And, for each $m, M_{n} \cap \mathrm{TB}_{n m} \cong$ $\subseteq M_{n}^{\prime} \cap T_{n}$ so that $\mu^{\prime}\left(M_{n}^{\prime} \cap T_{n m}\right) \leqq \mu^{\prime}\left(M_{n}^{\prime} \cap T_{n}\right)$. Hence $p_{n}=21 m_{m} \mu \cdot\left(M_{n}^{\prime} \cap T B_{n m}\right) \leqq \mu \cdot\left(M_{n}^{\prime} \cap T_{n}\right)$. From the definition of $p_{n}$ and from the fact that $B_{n}$ is in $\Gamma$ it follows that

$$
\mu \cdot\left(M_{n}^{\prime} \cap T B_{n}\right)=P_{n} \cdot
$$

Next we set $B_{0}=U_{n \geq 1} B_{n} \cdot B_{O}$ is in $\Gamma$ and, given $n \geqq 1$, suxely $M_{n}^{\prime} \cap \mathrm{TB}_{n} \subseteq M_{n}^{\prime} \cap \mathrm{TB}_{0}$ and so $\mu^{\prime}\left(M_{n}^{\prime} \cap \mathrm{TB}_{n}\right) \leqq \mu^{\prime}\left(M_{n}^{\prime} \cap \mathrm{TB}_{0}\right)$. It follows as before from the definition of $p_{n}$ that

$$
\mu \cdot\left(M_{n}^{\prime} \cap T B_{0}\right)=p_{n}, \quad n \geqq 1
$$


The set ${ }^{T B_{O}}$ is in $\underline{y}^{\prime}$. By $H 6$ there exists a set $B_{0}^{\prime}$ in $\underline{B}^{\prime}$ such that $\mathrm{TB}_{0} \subseteq \mathrm{B}_{0}^{\prime}$ and $\mu^{\prime}\left(\mathrm{B}_{0}^{\prime} \cap \mathrm{C}^{\prime} \mathrm{TB}_{0}\right)=0$. Define a non-negative function $W_{\#}$ on $\underline{S} \times \underline{D}$ by

$$
w_{\#}\left(s^{\prime}, D\right)=\left\{\begin{array}{cl}
W^{\prime}\left(s^{\prime}, D\right), & \left(s^{\prime}, D\right) \in C^{\prime} B_{0}^{\prime} \times \underline{D} \\
0, & \left(s^{\prime}, D\right) \in B_{0}^{\prime} \underline{D}
\end{array} .\right.
$$

By Lemma I.7.I W is a weight function for T. W' is almost over additive and so $W_{\#}$ is almost over additive. Hence $W_{\text {\# }}$ is over additive. Moreover $\mathrm{T}$ is $\mathrm{BVW}_{\#^{\bullet}}$ 'Define $\mathrm{S}_{\#_{+}}=\left\{_{\mathbf{s}^{\prime}} \in \underline{S}^{\prime}:\right.$ $\left.W_{\#}\left(B^{\prime}, \underline{s}\right)>0\right\}$. Note that $s_{\#+}=s_{+}^{\prime} \cap C^{\prime} B_{0}^{\prime}$ Note further that we shall be able to apply Theorem II.3.2 if we can verify the following statement:

$$
\text { If } B \text { is a ret in } \underline{B} \text { for which } B \subseteq T^{-1} S_{\#+} \text { and } \mu B=0
$$
then $\mu \cdot T B=0$.

In order to verify statement (A) we let $B$ be a set in $\underline{B}$ for which $B \subseteq \mathrm{T}^{-1} \mathrm{SH}_{\#+}$ and $\mu \mathrm{B}=0$. We assert

$$
\mu \cdot T B=0
$$

Fix a positive integer $n$. Surely $B$ is in $\Gamma$ and so $M_{n}^{\prime} \cap$ TB is in $\Gamma_{n}^{\prime}$. Note that

$$
\begin{aligned}
\mathrm{B} \subseteq \mathrm{T}^{-1} S_{\#+} & =T^{-1}\left(S_{+} \cap \mathrm{C}^{\prime} \mathrm{B}_{0}^{\prime}\right) \\
& =\mathrm{T}^{-1} S_{+} \cap \mathrm{T}^{-1} \mathrm{C}^{\prime} \mathrm{B}_{0} \\
& =\mathrm{T}^{-1} S_{+} \cap \mathrm{CT}^{-1} \mathrm{~B}_{0} \\
& \subseteq \mathrm{T}^{-1} S_{+} \cap \mathrm{CT}^{-I_{\mathrm{PB}_{0}}}
\end{aligned}
$$


In short $\mathrm{B} \cong \mathrm{CP}^{-1} \mathrm{~TB}_{0^{\circ}}$ Hence $\mathrm{TB} \cap \mathrm{TB}_{0}=\varnothing$. Consequently

$$
\left(M_{n}^{\prime} \cap T_{0}\right) \cap\left(M_{n}^{\prime} \cap T B\right)=\varnothing \text {. }
$$

Now $B_{O}$ and $B$ are in $\Gamma$ so that $B_{O} \cup B_{\text {is }}$ in $\Gamma$. As a result, $M_{n}^{\prime} \cap T\left(B_{O} \cup B\right)$ is in $\Gamma_{n}^{\prime}$. We have, in view of (2) and (4),

$$
\begin{aligned}
P_{n} & =\mu \cdot\left(M_{n}^{\prime} \cap T B_{0}\right) \\
& \leqq \mu \cdot\left(M_{n}^{\prime} \cap T B_{0}\right)+\mu \cdot\left(M_{n}^{\prime} \cap T B\right) \\
& \leqq \mu \cdot\left(M_{n}^{\prime} \cap T\left(B_{0} \cup B\right)\right) \\
& \leqq p_{n} \cdot
\end{aligned}
$$

Since $p_{n}$ is finite we have $\mu^{\prime}\left(M_{n}^{\prime} \cap T B\right)=0$. The integer $n$ was arbitrary and so (3) must hold. Hence statement (A) is valid. According to Theorem II.3.2 T is $\mathrm{ACW}^{*}$ Since $W^{\prime}$ is singular and since $W_{\#} \leqq W^{\prime}$ it follows that $W_{\#}$ is trivial. Hence $W_{\#}\left(s^{\prime}, \underline{S}\right)=0$ a.e. $\mu^{\prime}$ on $\underline{S}^{\prime}$. It now follows from the fact that $\mu^{\prime}\left(B_{0}^{\prime} \cap C^{\prime T B}{ }_{0}\right)=$ $=0$ and from the definition of $W_{\#}$ that $W^{\prime}\left(s^{\prime}, \underline{S}\right)=0$ a.e. $\mu^{\prime}$ on $\mathrm{C}^{\circ} \mathrm{TB}_{\mathrm{O}} \cdot$

We have proved that $B_{0}$ is a set in $\underline{B}$ for which $\mu B_{0}=0$ and $W^{\prime}\left(s^{\prime}, \underline{S}\right)=0$ a.e. $\mu^{\prime}$ on $\mathrm{C}^{\prime} \mathrm{TB}_{0} \cdot$ Hence the singular weight function $W \cdot i s$ strongly singular.

II.5.2. Theorem. Assume H9, H4*, H7*, HC1, and HC2. Assume that W' is almost over additive and that $T$ is BVW. Then every Lebesgue-type decomposition for W' is a strong Lebesgue- 
type decomposition for W'. Moreover the singular component of such a decomposition must be almost over additive.

Proof. Let $\left(W_{A}^{\prime}, W_{S}^{\prime}\right)$ be a Lebesgue-type decomposition for $W^{\prime}$. Then $W^{\prime}=W_{A}^{\prime}+W_{S}^{\prime}$; moreover $T$ is $A C W_{A}$ and $W_{S}^{\prime}$ is singular. Since $T$ is $B^{B V W}$ there exists a set $M^{\prime}$ in $M^{\prime}$ such that $\mu^{\prime} M^{\prime}=0$ and

$$
w_{\dot{A}}\left(s^{\prime}, D\right)<\infty, \quad s^{\prime} \in C^{\prime} M^{\prime}, D \in \underline{D} .
$$

We define two non-negative functions ${ }_{A}^{\prime}$ and $W_{S}^{\prime}$ on $\underline{S}^{\prime} \times \underline{D}$ by

$$
\begin{aligned}
& \sigma_{A}\left(s^{\prime}, D\right)=\left\{\begin{array}{cl}
w_{A^{\prime}}\left(s^{\prime}, D\right), & s^{\prime} \in C^{\prime} M^{\prime}, D \in \underline{D} \\
0, & s^{\prime} \in M^{\prime}, D \in \underline{D}
\end{array}\right. \\
& n_{S^{\prime}}\left(s^{\prime}, D\right)=\left\{\begin{array}{cl}
w_{S^{\prime}}\left(s^{\prime}, D\right), & s^{\prime} \in C^{\prime} M^{\prime}, D \in \underline{D} \\
W^{\prime}\left(s^{\prime}, D\right), & s^{\prime} \in M^{\prime}, D \in \underline{D}
\end{array}\right.
\end{aligned}
$$

By Lema $I .7 .1$ both $\theta_{A}$ and $\theta_{S}^{\prime}$ are weight functions for $T$.

\section{Moreover}

$$
W^{\prime}=\hat{A}_{\dot{A}}+\hat{D}_{\mathbf{S}} \cdot
$$

In view of the fact that $\mu^{\prime} M^{\prime}=0$ it follows from Lemma $I .7 .5$ that $T$ is $A C Q_{A}$ and that $\hat{Q}_{S}$ is singular. The weight function $W_{\dot{A}}$ is finite valued. By Lemma II.4.4 $\hat{W}_{S}$ must be almost over additive. Using Lemma $I .7 .5$ again we see that $W_{S}^{\prime}$ must be almost over additive. By Lemma II.5.1 $\mathrm{W}_{S}^{\prime}$ is strongly singular. Hence $\left(W_{A}, W_{S}^{\prime}\right)$ is a strong Lebesgue-type decomposition for $W^{\prime}$. 
II.5.3. Corollary. (The Strong Decomposition Theorem) Assume H9, H4*, H7*, and $\mathrm{HCl}$ - HC4. Assume that $\mathrm{T}$ is BVW and that $W^{\prime}$ is almost over additive. Then there exists a strong Lebesgue-type decomposition for W'. Its singular component is almost over additive.

Proof. By Theorem II.2.8 there exists a Lebesgue-type decomposition $\left(W_{A}^{\prime}, W_{S}^{\prime}\right)$ for $W^{\prime}$. But, by Theorem II.5.2, ( $\left.W_{A}^{\prime}, W_{S}^{\prime}\right)$ must be a strong Lebesgue-type decomposition for $W$ ' and $W_{S}^{\prime}$ must be almost over additive.

II.6 Almost additive weight functions

II.6.1. Remarks. Recently there has been an indication of some interest in almost additive weight functions. We now prove several basic results about such weight functions and establish a decomposition theorem for them.

\section{II.6.2. Lemma. Assume that $W^{\prime}$ is a non-negative function} defined on $\underline{S} \times \underline{D}$ which is inner continuous and almost additive. Then $W^{\prime}$ is almost under additive.

Proof. Let $\left\{D_{i}\right\}_{i=0}^{I}$ be a finite subset of $\underline{D}$ for which the sets $D_{i}, I \leqq i \leqq I$, are pairwise disjoint and $U_{i=1}^{I} D_{i} \cong D_{0} \cdot$ By Lemma II.1.7 there exists, for $i=1, \ldots$, a sequence of sets $D_{i j}, j \geqq l$, in $\underline{D}$ with the following properties:

i) $D_{i j} \subseteq D_{i} j+I, I \leqq i \leqq I, \quad j \geqq I$; 
ii) the set $\left\{D_{i f}\right\}_{i=1}^{I}$ is of type $\gamma, j \geqq 1$;

$$
\text { iii) } U_{j \geqq I} D_{i j}=D_{i}, \quad I \leqq i \leqq I \text {. }
$$

Now fix the positive integer $j$. The $\operatorname{set}\left\{D_{i j}\right\}_{i=1}^{I}$ consists of pairwise disjoint subsets of $D_{0}$, each of type $\gamma$. By Lemma II.1.8 there exists a D-partition $\left(\left\{D_{j k}^{\#}\right\}_{k \gg l}, Y_{j}, Z_{j}\right)$ of $D_{0}$ for which $\left\{D_{i j}\right\}_{i=1}^{I} \subseteq\left\{D_{j k}^{\#}\right\}_{k \gg I}$. We may as well assume that $D_{i j}=D_{j i}^{\#}$, $I \leqq i \leqq I$. By assumption $W^{\prime}$ is almost additive. Consequently.

$$
\sum_{k_{\underline{\prime}} I} W^{\prime}\left(s^{\prime}, D_{j k}^{\#}\right)=W^{\prime}\left(s^{\prime}, D_{0}\right) \quad \text { a.e. } \mu^{\prime} \text { on } \underline{S}^{\prime} \cdot
$$

Hence

$$
\begin{aligned}
& \sum_{i=1}^{I} w^{\prime}\left(s^{\prime}, D_{i j}\right)+\sum_{k \geqq I+1} W^{\prime}\left(s^{\prime}, D_{j k}^{\#}\right)=W^{\prime}\left(s^{\prime}, D_{0}\right) \\
& \text { a.e. } \mu^{\prime} \text { on } \underline{S}^{\prime} \text {. }
\end{aligned}
$$

Since $W$ is non-negative we have

$$
\sum_{i=1}^{I} W^{\prime}\left(s^{\prime}, D_{i j}\right) \leqq w^{\prime}\left(s^{\prime}, D_{0}\right) \text { a.e. } \mu^{\prime} \text { on } \underline{S}^{\prime} \cdot
$$

Statement (1) holds for every $f \geqq 1$. It follows from (1),

$i)$, ifi), and the inner continuity of $W$ that

$$
\sum_{i=1}^{I} w^{\prime}\left(s^{\prime}, D_{i}\right) \leqq w^{\prime}\left(s^{\prime}, D_{0}\right) \text { a.e. } \mu^{\prime} \text { on } \underline{S}^{\prime} \cdot
$$

Thus W' is almost under additive. 
II.6.3. Remark. It follows from Lemma II.6.2 that if W' satisfies conditions 1 ), $i i i)$, and $i v)$ for weight functions and If $W^{\prime}$ is almost additive then it is almost under additive. This fact does not guarantee that $W$ is a weight function for $T$. However there is reason to suspect that it may be possible to replace $i i)$ of $\mathrm{H} 9$ by the requirement that $W$ ' be almost under aditive without significantly altering the theory. If such a replacement were possible one could then say that a non-negative, almost additive function defined on $\underline{S} \times \underline{D}$ is a weight function for $T$ if it satisfies $i)$, iii), and $i v)$ of 89.

This matter remains unsettled. Thus, for the remainder of Section II.6, we shall require that W' be both a weight function for $T$ and an almost additive function. It must be remembered that later work may produce a redundancy in this requirement. (See Remark II.1.10.)

II.6.4. Lemma. Assume that $W^{\prime}$ is an almost additive weight function for $T$. Let $D_{i}, i \geqq 0$, be a countable family of sets in $D$ such that $D_{0} \subseteq U_{i \gg I^{1}} D_{i}$. Suppose that $D_{i}$ is of type $Y$ for $i \geqq 1$. Then

$$
W^{\prime}\left(s^{\prime}, D_{0}\right) \leqq \sum_{i \geqq I} W^{\prime}\left(s^{\prime}, D_{i}\right) \quad \text { a.e. } \mu^{\prime} \text { on } \underline{s}^{\prime} \cdot
$$

Proof. The conclusion follows from Lemma II.6.2 if there are only two sets, $D_{0}$ and $D_{1}$, involved. We shall assume then that at least three sets $-D_{0}, D_{1}, D_{2}$, and perhaps more - are 
involved. We define $E_{1}^{*}=D_{0} \cap D_{1}, E_{2}^{*}=D_{0} \cap C D_{1} \cap D_{2}$, and, in general,

$$
E_{k}^{*}=D_{0} \cap n_{i=1}^{k-1} C D_{i} \cap D_{k}, \quad k \geqq 1 \text {. }
$$

The sets $F_{k}^{*}, k \geqq I$, are pairwise disjoint and their union is $D_{0} \cdot D_{1}$ is of type $\gamma$ for $i \geqq l$. There exists a D-partition $\left(\left\{D_{i j}\right\}_{j \geqq 0}, Y_{i}, z_{i}\right)$ of $\underline{S}$ for which $D_{i} \in\left[D_{i j}\right\}_{j \geqq 0}$; let us suppose $D_{i}=D_{i 0^{\circ}}$ It is also possible to require that $Y_{i} U z_{i}=C U_{j \geqq 0} D_{i j}$ There exist sets $D_{1 p}, p \geqq 1$, in $\underline{D}$ which are pairwise disjoint and for which $E_{1}^{*}=D_{0} \cap D_{1}=U_{p \geqq 1} D_{1 p}$. Now fix an integer $k$ with $k \geqq 2$. Note that

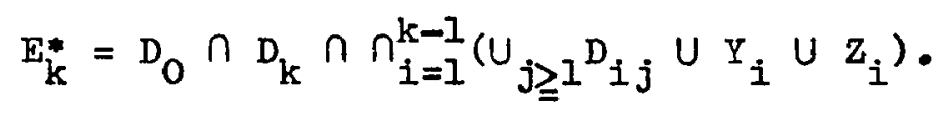

Let $J_{k-1}$ be the family of all sequences of positive integers of length $k-1$. Observe that

$$
D_{0} \cap D_{k} \cap U_{\left\{j_{I} \ldots j_{k-1}\right\} \in J_{k-1}}\left(\cap_{i=1}^{k-1} D_{i j_{1}}\right) \cong E_{k}^{*} \cdot
$$

Also

$$
\begin{aligned}
& \mathbf{E}_{k}^{*} \cong\left[D_{0} \cap D_{k} \cap U_{\left(j_{1} \ldots j_{k-1}\right\} \in J_{k-1}}\left(\cap_{i=1}^{k-1} D_{i j_{i}}\right)\right] U \\
& U U_{i=1}^{k-1}\left(Y_{i} \cup z_{i}\right) .
\end{aligned}
$$

For each $\left\{j_{1} \ldots j_{k-1}\right\}$ in $J_{k-1}$ there is by Lemma 8.2 of [2] a countable family of pairwise disjoint sets $D_{p}^{\left(f_{1} \cdots J_{k-1}\right\}}, p \geqq 1$, 
in $D$ for which $D_{0} \cap D_{k} \cap \cap_{i=1}^{k-1} D_{i j_{i}}=U_{p \geq I} D_{p}^{\left\{f_{1} \cdots \cdots j_{k-1}\right\}}$. In view of (1) we have

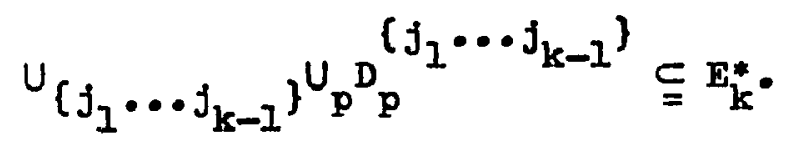

(2) becomes

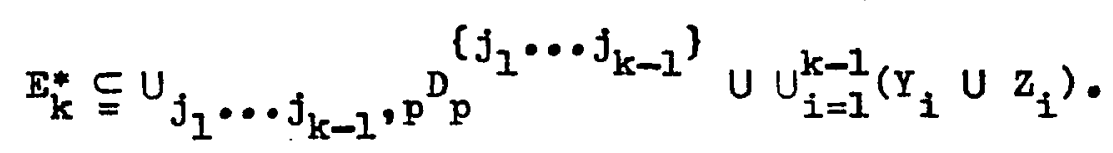

The sets in the family $\left(D_{p}^{\left\{j_{1} \cdots j_{k-1}\right\}}: p \geqq 1,\left\{j_{1} \cdots j_{k-1}\right\} \in J_{k-1}\right\}$ are evidently pairwise disjoint. Since the sets $E_{k}^{*}, k \geqq l$, are pairwise disjoint it now follows from (3) and from the last sentence of the preceding paragraph that the sets in the family $\underline{D}_{0}=\left\{D_{1 p}\right\} p l \mid$ $U\left\{D_{p}^{\left(j_{1} \cdots j_{k-1}\right\}}: p \geqq 1,\left\{j_{1} \ldots j_{k-1}\right\} \in J_{k-1}\right\}_{k \geq 2}$ are pairwise disjoint. Moreover we obtain

$$
\begin{aligned}
& U_{p \geqq 1} D_{1 p} \cup U_{k \geqq 2}\left[U_{\left\{j_{1} \ldots j_{k-1}\right\}, p_{p}} D^{\left(j_{1} \cdots j_{k-1}\right\}}\right] \subseteq \\
& \subseteq U_{k \geqq 1} F_{k}^{*}=D_{0} .
\end{aligned}
$$

Using (4) we obtain $D_{0}=U_{k \geqq 1} E_{k}^{*} \leqq U_{p \geqq 1} D_{1 p} U$

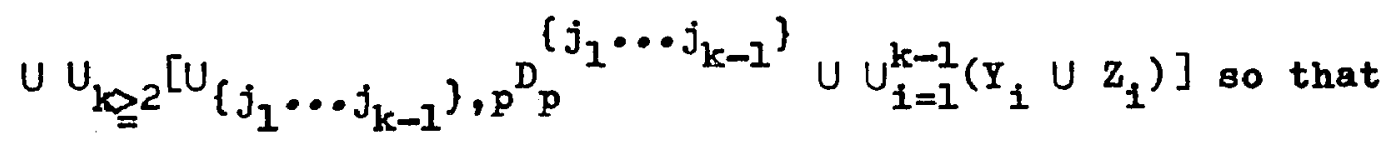




$$
\begin{aligned}
& \left.D_{0} \subseteq U_{p \geqq I} D_{1 p} U U_{k \geqq 2} U\left(j_{1} \ldots j_{k-1}\right\}, p^{D_{p}}\left[j_{1} \cdots j_{k-1}\right\}\right) \\
& U U_{k \geqq I}\left(Y_{k} U z_{k}\right) .
\end{aligned}
$$

From (6) and (5) it follows that

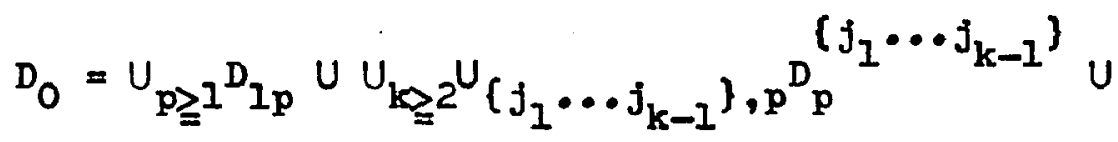

$$
\begin{aligned}
& U U_{k \geq I}\left(D_{0} \cap Y_{k}\right) \cup U_{k \geqq l}\left(D_{0} \cap z_{k}\right) \text {. }
\end{aligned}
$$

Note that $U_{k \geq I}\left(D_{0} \cap Y_{k}\right)$ is in $\underline{M}$ and

$$
\mu U_{k \geqq I}\left(D_{0} \cap Y_{k}\right)=0 \text {. }
$$

Similarly $T U_{k \gtrless l}\left(D_{0} \cap z_{k}\right)$ is in $M^{\prime}$ and

$$
\mu \cdot T U_{k \geq I}\left(D_{0} \cap z_{k}\right)=0
$$

It now follows from (7), (8), (9), and the first sentence of this paragraph that $\left(\underline{D}_{\square}, U_{k \geq I}\left(D_{0} \cap Y_{k}\right), U_{k \geqq l}\left(D_{0} \cap z_{k}\right)\right)$ is a $\underline{D}-$ partition of $D_{0}$. Since $W '$ is almost additive we have

$$
\begin{aligned}
& W^{\prime}\left(s^{\prime}, D_{0}\right)=\sum_{p \geq I^{\prime}} W^{\prime}\left(s^{\prime}, D_{l p}\right)+ \\
& +\sum_{k \geq 2} \sum_{\left\{j_{1} \ldots j_{k-1}\right\}, p} W^{\prime}\left(s^{\prime}, D_{p}\left\{j_{1} \ldots j_{k-1}\right\}\right) \text { a.e. } \mu^{\prime} \text { on } \underline{s}^{\prime} \cdot
\end{aligned}
$$

The sets $D_{1 p}, p \geqq 1$, are pairwise disjoint subsets of $D_{1}$ so that, in view of the under additivity of $W$ ', we have 
(11)

$$
\sum_{p \geqq 1} W^{\prime}\left(s^{\prime}, D_{1 p}\right) \leqq W^{\prime}\left(s^{\prime}, D_{1}\right), s^{\prime} \in \underline{S}^{\prime} \cdot
$$

Similarly we obtain

$$
\begin{aligned}
& \sum_{\left\{j_{1} \ldots j_{k-1}\right\}, p} w^{\prime}\left(s^{\prime}, D_{p}^{\left(j_{1} \cdots j_{k-1}\right\}}\right) \leqq w^{\prime}\left(s^{\prime}, D_{k}\right),
\end{aligned}
$$

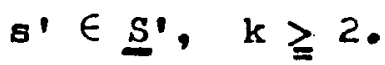

Combining (10), (11), and (12) yields

$$
W^{\prime}\left(s^{\prime}, D_{0}\right) \leqq W^{\prime}\left(s^{\prime}, D_{1}\right)+\sum_{k_{\underline{Z}}} W^{\prime}\left(s^{\prime}, D_{k}\right) \text { a.e. } \mu^{\prime} \text { on } \underline{S}^{\prime} \cdot
$$

II.6.5. Lemma. Assume HC2. Assume that $W '$ is an almost additive weight function for $T$. Then $W$ ' is almost over additive.

Proof. Let $D^{j}, j \geqq 0$, be a countable family of sets in $\underline{D}$

for which $D^{0} \subseteq U_{J \geqq I} D^{j}$. By HC2 there exists a sequence of sets $D_{i}^{\prime}, i \geqq I$, in $D$ with the following properties:

i) $D_{i} \cong D_{i+1}, \quad i \geqq 1$;

ii) $D^{0}=U_{i \geqq 1} D_{i}$;

iii) if $\#^{j}, j \geqq 1$, is any countable family of sets in D for which $D^{0} \subseteq U_{j \geqq 1} \#^{j}$ then for every positive integer $i$ there exists a positive integer $J(i)$ such that $D_{i} \subseteq U_{j=1}^{j(i)} \#_{D^{j}}$. 
By $\mathrm{H} 5$ there is for $j \geqq 1$ a sequence of $\operatorname{sets} D^{j p}, p \geqq 1$, in $\underline{D}$ with the following properties:
a) $D^{j p} \cong D^{j p+1}, p \geqq 1 ;$
b) $D^{j}=U_{p \geqq I^{j p}}$;
c) each $D^{j p}$ is of type $\gamma$.

Observe that $D^{0} \subseteq U_{j \geqq 1} D^{j}=U_{j, p} D^{j p}$.

At this point we fix the positive integer $i$. By $i i i)$ there exist positive integers $P(i)$ and $J(i)$ such that $D_{i} \subseteq U_{j=1}^{J(i) P(i)} D^{j p}$. Using a) we obtain $D_{i} \cong U_{j=1}^{J(i)} D^{j P(i)}$. Each $D^{j P(i)}$ is of type $\gamma$ and $W^{\prime}$ is almost additive; by Lemma II.6.4

$$
W^{\cdot}\left(s^{\prime}, D_{i}\right) \leqq \sum_{j=1}^{J(i)} W^{\prime}\left(s^{\prime}, D^{j P(i)}\right) \text { a.e. } \mu^{\prime} \text { on } \underline{S}^{\prime} \cdot
$$

Using $b$ ) and the under additivity of $W$ ' we deduce from (1)

$$
W^{\prime}\left(s^{\prime}, D_{i}\right) \leqq \sum_{j=1}^{J(i)} w^{\prime}\left(s^{\prime}, D^{j}\right) \text { a.e. } \mu^{\prime} \text { on } \underline{s}^{\prime} \cdot
$$

In view of (2), we see that we must surely have

$$
W^{\prime}\left(s^{\prime}, D_{i}\right) \leqq \sum_{j \geqq I} W^{\prime}\left(s^{\prime}, D^{j}\right) \text { a.e. } \mu^{\prime} \text { on } \underline{S}^{\prime} \cdot
$$

Statement (3) holds for every positive integer $i$. In view of $(3), i), 1 i)$, and the inner continuity of $W^{\prime}$ we have 


$$
W^{\prime}\left(s^{\prime}, D^{0}\right) \leqq \sum_{j \geqq 1} W^{\prime}\left(s^{\prime}, D^{j}\right) \quad \text { a.e. } \mu^{\prime} \text { on } \underline{s}^{\prime} \cdot
$$

Hence $W$ is almost over additive.

II.6.6. The following decomposition theorem now becomes a corollary of Theorem II.5.2 and Corollary II.5.3.

Theorem. Assume H9, H4*, H7*, HCl, and HC2. Assume that $W$ is almost additive and that $T$ is $B V W$.

1) Then every Lebesgue-type decomposition for W' is a strong Lebesgue-type decomposition for W'. The singular component of such a decomposition must also be almost additive.

2) Assume also HC4. Then there exists a strong Lebesguetype decomposition for W'. Its singular component is almost additive.

Proof. First we prove I). By Lemma II.6.5 W' is almost over additive. From Theorem II.5.2 it then follows that every Lebesgue-type decomposition for $W^{\prime}$ is a strong Lebesgue-type decomposition for W'. To see that the singular component of such a decomposition must be almost additive, let $\left(W_{A}, W_{S}\right)$ be a Lebesgue-type decomposition for W'. W' is almost additive and, by Lemma 4.1 of $[2], W_{A}$ is almost additive. Recall that $W^{\prime}=W_{A}^{\prime}+W_{S}^{\prime} ;$ it follows at once that $W_{S}^{\prime}$ is also almost additive. 2) follows at once from Corollary II.5.3, the fact that W' is almost over additive, and from 1 ). 
II.6.7. Remarks. The decomposition theorem just proved for almost additive weight functions is essentially a mere special case of the results of section II.5. It is of interest only because of the potential importance of almost additive weight functions. In Lemwa II.6.5 we proved that an almost additive weight function is almost over additive (if $\mathrm{HC2}$ is satisfied). One might ask whether an almost over additive weight function is necessarily almost additive. This question is answered in Section II.7.

\section{II.7 Examples}

II.2.1. Remarks. In this section we present several examples in order to illuminate a few points. The examples presented here are not the result of a systematic search. This section is not intended to be a comprehensive list of known examples. Indeed these examples were discovered rather incidentally. They do however serve as counter-examples for several interesting conjectures. It should be noted that Theorems III.2.3 and III.3.4 are used in this section. None of the results of Chapter III is dependent upon this section. It should also be noted that no a priori assumptions are made in this section.

II.7.2. Example. Let $\underline{S}$ be a non-empty bounded domain in $R^{2}$ and let $\pi$ denote the projection from $\underline{S}$ into the "X-axis"; $\pi$ is defined by $\pi[(x, y)]=x,(x, y) \in \underline{S}$. Set $\underline{S}^{\prime}=\pi \underline{S}$. Let $I_{1}$ denote one-dimensional Lebesgue measure and let $\mathrm{L}_{2}$ denote. 
two-dimensional Lebergue measure. Let $M$ be the family of all $I_{2}$-measurable sets which are subsets of $\underline{S}$ and let $\underline{M}^{\prime}$ be the family of all $I_{1}$-measurable sets which are subsets of $\underline{S}^{\prime} \cdot$ Set $\mu=I_{2} \mid \underline{M}$ and $\mu^{\prime}=I_{1} \mid \underline{M}^{\prime}$. Let $\underline{D}$ be the family of all subsets of S which are domains. Suppose $\underline{B}^{\prime}$ is the family of all Borel sets in $R^{1}$ which are subsets of $\underline{S}^{\prime}$. According to 9. of [2], the measure spaces $(\underline{S}, \underline{M}, \mu)$ and $\left\{\underline{S}^{\prime}, \underline{M}^{\prime}, \mu^{\prime}\right\}$, the families D and $B^{\prime}$, and the continuous map $\pi$ satisfy HI - H8, H4*, H7*, HIO - HI2, and H15. It follows from well-known theorems that HC2 - HC4 hold. Indeed if we define $\underline{D}_{0}$ to be the family of all domains $D$ in $D$ each of which is a 2-dimensional polyhedron having rational vertices then if we set $\underline{D}^{*}=\underline{D}^{*}=\underline{D}_{\#}=\underline{D}_{*}=\underline{D}_{0}$ we see that HIO, H15, HC3, and HC4 are all satisfied. Moreover, define $\underline{B}$ to be the family of all Borel sets in $R^{2}$ which are subsets of $\underline{S}$. It follows again from well-known results that HCl is satisfied. Thus all of the theory developed in [2] and in the present work can be applied here.

Now we define a weight function for $\pi$. Let $K$ be a fixed positive number for which $\underline{g}\left\{(x, y) \in R^{2}:|x| \leqq K,|y| \leqq K\right\}$. Thus

$$
\mu \underline{S}=I_{2} \underline{S} \leqq 4 K^{2}
$$

and

$$
\mu^{\prime} \underline{S}^{\prime}=I_{I} \underline{S}^{\prime} \leqq 2 K \text {. }
$$

For each $s^{\prime}$ in $\underline{S}^{\prime}$ and open subset $G$ of $\underline{S}$ the set $G_{g^{\prime}}$ defined by 
$G_{s^{\prime}}=\left\{y \in R^{l}:\left(s^{\prime}, y\right) \in G\right\}$ is an open set of real numbers and hence is surely $I_{1}$-measurable. Moreover

$$
I_{I}\left(G_{S^{\prime}}\right) \leqq 2 \pi
$$

We define a non-negative function $W^{\prime}$ on $\underline{S}^{\prime} \times \underline{D}$ by $W^{\prime}\left(s^{\prime}, D\right)=I_{1}\left(D_{S^{\prime}}\right)$, $\left(s^{\prime}, D\right) \in \underline{S^{\prime}} \times \underline{D}$. In view of $(3) W^{\prime}$ is bounded on $\underline{S}^{\prime} \times \underline{D}$. In order to show that $W$ ' is a weight function for $\pi$ we must check the four conditions of H9. First, we note that if $D$ is in $D$ and $s^{\prime}$ is in $C^{\prime} \pi D$ then $D_{s^{\prime}}=\varnothing$ and so $W^{\prime}\left(s^{\prime}, D\right)=0$. Using basic properties of measures (applied here for $L_{1}$ ) we see that $W^{\prime}$ is under additive and inner continuous; it is helpful to note that $G_{15}, \cap G_{2 s},=\varnothing$ when $G_{1} \cap G_{2}=\varnothing$. Next, fix $D$ in D. It follows from the Fubini Theorem for the plane that the function $W^{\prime}\left(B^{\prime}, D\right)=$ $=I_{I}\left(D_{s_{1}}\right), s^{\prime} \in \underline{S}^{\prime}$, is $\underline{M}^{\prime}$-measurable. Hence $W^{\prime}$ is a weight function for $\pi$. As usual, we let $W D, D \in \underline{D}$, be the weights induced by W'. We apply the Fubint Theorem again to obtain $W D=\mathcal{S}_{S^{\prime}} W^{\prime}\left(s^{\prime}, D\right) d \mu \prime=L_{2} D=\mu D, D \in \underline{D}$, or, briefly,

$$
W D=\mu D, \quad D \in \underline{D} .
$$

Using (4) and (I) we obtain WS $=\mu \underline{S} \leqq 4 K^{2}$. Hence $\pi$ is BVW. Moreover if we define a non-negative function $f_{1}$ on $\underline{S}$ by $f_{1}(s)=$ $=I, s \in \underline{S}$, it follows from (4) that $W D=\int_{D} f_{I}(s) d \mu, D \in \underline{D}$. Hence $\pi$ is ACW. We shall now use this example to discuss a possible extension of one theorem and the converse of another. Theorem 8.9 of [2] asserts that if HI - HI2, HI4, and HI5 
are satisfied and if $T$ is ACW then $W$ "essentlally" satisfies H13. In our present setting all of the hypotheses of Theorem 8.9 of [2] are satisfied except HI4. We shall now show that the conclusion of Theorem 8.9 is not satisfied in the present situation; that 1s, we shall show that if a non-negative function - is defined on 5 by

$$
A S=\inf [W \cdot(\pi S, D): S \subseteq D \in \underline{D}\}, \quad S \in S,
$$

then it is false that

$$
W \cdot\left(B^{\prime}, D\right)=\Sigma A S, S \in S_{B}, S \subseteq D_{1}
$$

a.e. $\mu^{\prime}$ on $\underline{S}^{\prime}$ uniformly with respect to $\underline{D}$. To see this we shall show is identically zero on 5 .

Now according to 9. of [2] the family 5 consists of those subsets of $S$ which contain exactly one point. Pick $S_{0}$ in 5 . The set $S_{0}$ consists of a single point $s_{0}$. Define for all $r>0$ the set $s\left(s_{0}, r\right)$ by

$$
s\left(s_{0}, r\right)=\left\{(x, y) \in R^{2}: d_{2}\left[(x, y), s_{0}\right]<r\right\}
$$

Here, $d_{2}$ is the conventional Euclidean metric for $R^{2}$. For all integers $n$ which are sufficiently large, say $n \geqq N_{0}$, the set $D^{n}=S\left(s_{0}, \frac{I}{n}\right)$ is a subset of $\underline{S}$ and hence an element of $\underline{D}$. Whenever $n \geqq N_{0}$ we have $W^{\prime}\left(\pi s_{0}, D^{n}\right)=L_{I}\left(D_{\pi s_{0}}^{n}\right)=\frac{2}{n}$; but also $W^{\prime}\left(\pi s_{0}, D^{n}\right) \geqq Q S_{O}$. Hence $Q S_{0}=0$. Hence $Q$ is identically zero on 5. Since it is false that $W^{\prime}=0$ a.e. $\mu^{\prime}$ on $\underline{S}^{\prime}$ uniformly 
with respect to D it is clear that the conclusion of Theorem 8.9 does not hold here. The requirement that HI4 hold cannot therefore be dropped entirely from Theorem 8.9 without making some sort of replacement.

The following statement is a converse to Theorem II.3.2: Assume HI - H9, H7*, and HCl. Assume that T is ACW. Then if $B$ is a set in $B$ such that $\mu B=0$ and $B \subseteq T^{-1}\left\{s^{\prime} \in \underline{S}^{\prime}: W^{\prime}\left(s^{\prime}, \underline{S}\right)>0\right\}$ it is true that $\mu \cdot T B=0$

We can use the present example to show that this statement is false. It is conrenient to make a definite choice for $\underline{S}$. We define $\underline{S}=\left\{(x, y) \in R^{2}: x^{2}+(y-2)^{2}<1\right\}$. We thereby obtain a special case of the example just presented. The hypotheses of the above "converse" are fulfilled since $\pi$ is ACW. And yet, the conclusion is false. To see this, set $\lambda=\{(x, y) \in \underline{S}: y=2\}$. Then $\lambda$ is in $\underline{B}, \lambda \subseteq \pi^{-1}\left\{s^{\prime} \in \underline{S}^{\prime}: w^{\prime}\left(s^{\prime}, \underline{S}\right)>0\right\}$, and $\mu \lambda=0$. But $\mu \cdot \pi \lambda=I_{1}[(-1,1)]=2$.

II.7.3. Example. In Section II.6 we investigated almost additive weight functions. We proved that (under certain conditions) every almost additive weight function is almost over additive. One might ask whether every almost over additive weight function is almost additive. One might also ask whether every weight function satisfying Hl3 is almost additive. The answers to these questions become clear from the following example. 
In this example our choices for $\underline{S}, \underline{S}^{\prime}, \underline{M}, \underline{M}^{\prime}, \mu, \mu^{\prime}, \pi, \underline{D}$, $\underline{B}$, and $\underline{B}^{\prime}$ are the same as those made in Example II.7.2. However we again make a special choice for $\underline{S}$. In fact we again set $\underline{S}=\left\{(x, y) \in R^{2}: x^{2}+(y-2)^{2}<1\right\}$. Then $\underline{S}^{\prime}=(-1,1)$. Again

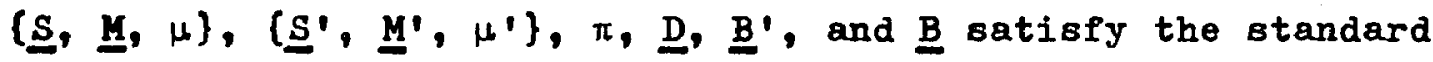
hypotheses HI - H8, H4*, Ḧ*, HIO - HI2, HI5, and HCl - HC4. Indeed the only departure from the last example is found in the choice of the weight function for $\pi$. Here we shall introduce a weight function which is generated by a non-negative function w defined on 5. Again define $\lambda=\{(x, y) \in \underline{S}: y=2\}$. Again $\lambda$ is in B. As before, 5 consists of all those subsets of $S$ which contain exactly one point; as a result, either $s \subseteq \lambda$ or $s \cap \lambda=\varnothing$

whenever $S$ is in 5 . We define a non-negative function $w$ on $S$ by

$$
w S=\left\{\begin{array}{l}
I, \quad s \subseteq \lambda \\
0, \quad s \cap \lambda=\varnothing^{\circ}
\end{array}\right.
$$

Because $\lambda$ is in $B$ it follows at once from Theorem III.2.3 that the summatory function $W_{*}^{\prime}\left(s^{\prime}, D, W\right),\left(s^{\prime}, D\right) \in \underline{S}^{\prime} \times \underline{D}$, is a weight function for $\pi$. This weight function satisfies H13 (by construction) and hence is over additive (7.8 of [2]).

We now assert that this weight function is not almost additive. Define $D_{1}=\{(x, y) \in \underline{S}: y>2\}$ and $D_{2}=\{(x, y) \in \underline{S}$ :

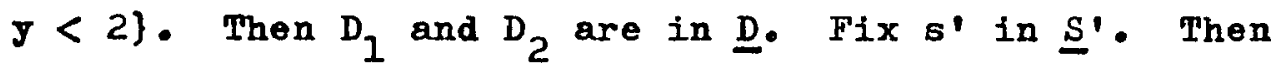

$$
W:\left(s^{\prime}, \underline{S}, w\right)=\Sigma w S, \quad S \subseteq \pi^{-1} s^{\prime}, \quad=w\left\{\left(s^{\prime}, 2\right)\right\}=1
$$

while also 


$$
W_{*}\left(s^{\prime}, D_{i}, w\right)=\Sigma w S, \quad s \in \sigma_{g^{\prime}}, S \subseteq D_{i},=0, \quad 1=1,2 .
$$

Since $\mu \lambda=0$ and $\underline{S}=D_{1} \cup D_{2} \cup \lambda$ it is clear that $\left(\left\{D_{1}\right\}_{1=1}^{2}, \lambda, \varnothing\right)$ is a $\underline{\text { D-partition of }}$ S. Thus if $W_{*}$ were almost additive we would then have

$$
w_{*}^{\prime}\left(s^{\prime}, \underline{S}, w\right)=w_{*}\left(s^{\prime}, D_{1}, w\right)+w_{*}^{\prime}\left(s^{\prime}, D_{2}, w\right) \text { a.e. } \mu^{\prime} \text { on } \underline{S}^{\prime} \cdot
$$

(1) and (2) hold for every $s^{\prime}$ in $S^{\prime}$. Hence (3) cannot hold unless $\mu^{\prime} \underline{S}^{\prime}=0$. But $\mu^{\prime} \underline{S}^{\prime}=2$. Hence $W$ : cannot be almost aditive.

We have exhibited a weight function which is (almost) over aditive and which even satisfies $H 13$ and yet is not almost additive.

II.7.4. The following theorem gives three methods by which new weight functions can be constructed from given ones.

Theorem. Assume HI - H8. Assume W' is a weight function for T. Let $t$ be a positive real number.

1) Define a non-negative function $t^{\prime \prime}$ on $\underline{S}^{\prime} \times \underline{D}$ by

$$
t^{\prime}\left(s^{\prime}, D\right)=\left\{\begin{array}{cl}
W^{\prime}\left(s^{\prime}, D\right), & \text { if } W^{\prime}\left(s^{\prime}, D\right)>t \\
0, & \text { if } W^{\prime}\left(s^{\prime}, D\right) \leq t
\end{array},\left(s^{\prime}, D\right) \in \underline{s}^{\prime} \times \underline{D} .\right.
$$

Then $0 \leqq W^{\prime} \leqq W^{\prime}$ and $t^{\prime \prime}$ is a weight function for $T$.

2) Define a non-negative function $w_{t}^{\prime}$ on $\underline{S}^{\prime} \times \underline{D}$ by

$$
W_{t}^{\prime}\left(s^{\prime}, D\right)=\left\{\begin{array}{cc}
W^{\prime}\left(s^{\prime}, D\right), & \text { if } \mu D>t \\
0, & \text { if } \mu D \leqq t
\end{array},\left(s^{\prime}, D\right) \in \underline{S}^{\prime} \times \underline{D} .\right.
$$

Then $0 \leqq W_{t}^{\prime} \leqq W^{\prime}$ and $W_{t}^{\prime}$ is a weight function for $T$. 
3) Define a non-negative function $w_{t T}$ on $\underline{S} \times \underline{D}$ by

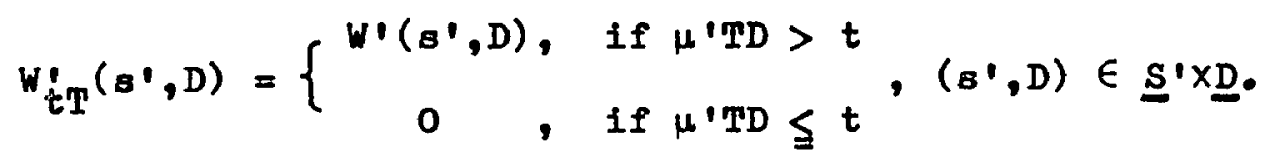

Then $0 \leqq W_{t T} \leqq W^{\prime}$ and $W_{t T}$ is a weight function for $T$.

Proof. In each case the proof consists of a straightforward verification of i) - iv) of H9. The details are omitted. Note that the weight function $t^{W}$ introduced in 1 ) always satisfies HבI.

Parts 2) and 3) of Theorem II.7.4 are due to Reichelderfer.

II.7.5. Example. Using 2) of Theorem II.7.4 it is easy to construct an example which reveals that two conjectures are false. We set $\underline{S}=\left\{s \in R^{1}: 0<s<1\right\}$ and $\underline{S}^{\prime}=\left\{s^{\prime} \in R^{1}: 0<s^{\prime}<I\right\}$. Let $\underline{M}$ consist of all $I_{1}$-measurable sets which are subsets of $\underline{S}$ and let $M^{\prime}$ consist of all $I_{1}$-measurable sets which are subsets of $\underline{S}^{\prime}$. Set $\mu=I_{1} \mid \underline{M}$ and $\mu^{\prime}=L_{1} \mid \underline{M}{ }^{\prime}$. Define the mapping $T$ from $\underline{S}$ into $\underline{S}$ by $T s=s, s \in \underline{S} . \quad T$ is the identity mapping from $\underline{S}$ onto $\underline{S}^{\prime}=\underline{S}$. Let $\underline{D}$ consist of all domains in $(0,1)$ and let $\underline{B}$ and $\underline{B}^{\prime}$ be the families of all Borel sets in $R^{l}$ which are subsets of $\underline{S}$ and $\underline{S}^{\prime}$ respectively. According to 9. of $[2],\{\underline{S}, \underline{M}, \mu\}$, $\left\{\underline{S}^{\prime}, \underline{M}^{\prime}, \mu^{\prime}\right\}, T, \underline{D}, \underline{B}^{\prime}$, and $\underline{B}$ satisfy HI - H8, HlO - Hl2, Hl5, H4*, and $H 7^{*}$. It also follows from well-known theorems that HCl - HC4 are satisfied.

The family 5 consists of those subsets of $\underline{S}=(0,1)$ which contain exactly one point. Define a non-negative function w 
on 5 by $w S=1, S \in 5$. By Theorem III.2.3 the function $W^{\prime}$ defined on $\underline{S} \times \underline{D}$ by

$$
W^{\prime}\left(s^{\prime}, D\right)=W^{\prime}\left(s^{\prime}, D, W\right), \quad\left(s^{\prime}, D\right) \in \underline{S}^{\prime} \times \underline{D},
$$

is a weight function for $T$. We now introduce the non-negative function $W_{i / 2}$ defined on $\underline{S} \times \underline{D}$ by

$$
W_{i / 2}\left(s^{\prime}, D\right)=\left\{\begin{array}{cl}
W^{\prime}\left(s^{\prime}, D\right), & \text { if } \mu D>\frac{I}{2} \\
0, & \text { if } \mu D \leqq \frac{I}{2}
\end{array},\left(s^{\prime}, D\right) \in \underline{S}^{\prime} \times D .\right.
$$

By 2) of Theorem II.7.4 $\mathrm{W}_{\mathrm{i} / 2}$ is a weight function for T. We develop this example further and state several of its consequences in a series of remarks.

i) $W D=\mu D=\mu \cdot T D, D \in \underline{D}$.

Proof. $W^{\prime}\left(s^{\prime}, D\right)=I$ if $s^{\prime}$ is in TD and is 0 if $s^{\prime}$ is in C'TD. Hence WD $=\mu \cdot T D$. But also $\mu \cdot T D=L_{1} D=\mu D$.

ii) $W_{1 / 2} \underline{S}=1$. Hence $T$ is $B_{1 / 2^{*}}$

Proof. Observe that $w_{1 / 2} \underline{S}=\int_{\underline{S}} \cdot w_{i / 2}^{\prime}\left(s^{\prime}, \underline{S}\right) d \mu^{\prime}=$ $=\int_{S^{\prime}} W^{\prime}\left(s^{\prime}, \underline{S}\right) d \mu^{\prime}=W \underline{S}=1$.

iii) Suppose W' is a weight function for $T$ for which $w_{0} \leqq w_{i / 2}$ and $w_{0}$ is over additive. Then $w_{0}=0$.

Proof. The sets $D_{1}=\left\{s \in \underline{s}: 0<s<\frac{1}{2}\right\}, D_{2}=\{s \in \underline{s}:$

$\left.\frac{1}{4}<s<\frac{3}{4}\right\}$, and $D_{3}=\left\{s \in \underline{s}: \frac{1}{2}<s<1\right\}$ are in $\underline{D}$. Evidently $w_{i / 2}\left(s^{\prime}, D_{i}\right)=0, s^{\prime} \in \underline{s}^{\prime}$, for $i=1,2,3$. Hence $w_{1 / 2} D_{i}=0$, for $i=1,2,3$. Now $w_{0} \leqq w_{1 / 2}$ so that $w_{0} D_{i}=0$ for $i=1,2,3$. Since 
$\underline{S}=D_{1} \cup D_{2} \cup D_{3}$ and $W_{0}$ is over additive it follows that $W_{0} \underline{S}=0$. But then $w_{0}=0$.

iv) For every $\varepsilon>0$ there exists $\delta(\varepsilon)>0$ such that $\mu^{\prime} \mathrm{TB}<\varepsilon$ whenever $B$ is in $\underline{B}$ and $\mu B<\delta(\varepsilon)$.

Proof. Note that $\mu{ }^{\prime T B}=\mu B$ for all $B$ in $\underline{B}$. Set $\delta(\varepsilon)=\varepsilon$, $\varepsilon>0$.

$$
\text { v) It follows from } i i \text { ) and iii) that } w_{1 / 2} \text { is not over }
$$

additive. By 5.11 of [2] $\mathrm{T}$ is not $\mathrm{ACW}_{1 / 2^{\circ}}$ The following conjecture mast therefore be false; for its hypotheses are satisfied in the present example and yet the conclusion is false here:

Confecture. Assume $\mathrm{HI}-\mathrm{HI} 2, \mathrm{HI} 4, \mathrm{HI} 5, \mathrm{H}^{* *}, \mathrm{H} 7^{*}$, and $\mathrm{HCl}-$ HC4. Suppose that for every $\varepsilon>0$ there exists $\delta(\varepsilon)>0$ such that $\mu$ 'TB $<\varepsilon$ whenever $B$ is in $\underline{B}$ and $\mu B<\delta(\varepsilon)$. Assume also that $\mathrm{T}$ is $\mathrm{BVW}$. Then $\mathrm{T}$ is ACW.

vi) $W$ and $W_{1 / 2}$ are $A C$ with respect to $\mu$.

Proof. Since $W_{1 / 2} \leqq W$ it suffices to show that, $W$ is $A C$ with reapect to $\mu$. Thus suppose $M_{0}$ is a set in $\underline{M}$ with $\mu M_{0}=0$. Let $8>0$ be given. There is a countable number of pairwise dis-

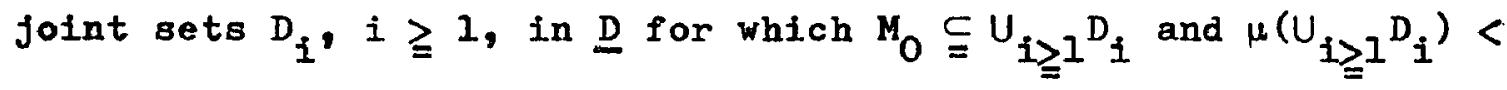
<.. By i) $W D_{i}=\mu D_{i}, i \geqq 1$. Hence $\Sigma_{i \geqq l} W D_{i}=\Sigma_{i \geqq I} \mu D_{i}<\varepsilon$. vii) $w_{i / 2}$ is singular. 
Proof. Suppose $W_{A}$ is an AC part of $w_{i / 2}$. By 5.11 of [2] $W_{A}$ is over additive. By iii), $W_{A}=0$.

viii) Let $W_{A}$ and $W_{S}^{\prime}$ be weight functions for $T$ for which $W_{A}^{\prime}+W_{S}^{\prime} \leqq W_{i / 2}, T$ is $A_{A} W_{A}$, and $W_{S}^{\prime}$ is strongly singular. Then $w_{A}=w_{S}=0$.

Proof. By vii) $W_{A}=0$. Since $w_{S}^{\prime}$ is strongly singular there is a set $B_{0}$ in $\underline{B}$ such that $\mu B_{0}=0$ and $W_{S^{\prime}}^{\prime}\left(B^{\prime}, \underline{S}\right)=0$ a.e. $\mu^{\prime}$ on $C^{\prime}{ }^{M B} B_{0}$. Note that $\mu \cdot{ }^{\prime} B_{0}=\mu B_{0}=0$. Hence $w_{S}^{\prime}\left(s^{\prime}, \underline{S}\right)=0$ a.e. $\mu^{\prime}$ on $\underline{S}^{\prime}$. Hence $W_{S}=0$. ix) There is no strong Lebesgue-type decomposition for $w_{i / 2}$

Proof. Use ii) and viii).

Thus there are weight functions for which there is no strong Lebesgue-type decomposition. We cannot hope to remove the requirement that $W$ ' be almost over additive from Corollary II.5.3 without making some sort of replacement.

II.7.6. Example. One may ask whether the classical Lebesgue decomposition theorem (see [3]) appears as a special case of our results. This classical theorem can be obtained from the Special Decomposition Theorem (III.3.4) but not in its most general form. Certain assumptions are required which are unnecessary in the customary approaches to the theorem. The rather awkward discussion follows. 
Assume that $\{X, \underline{M}, \mu)$ and $\{X, \underline{N}, \nu\}$ are measure spaces. Suppose that $\mu$ and $\nu$ are finite valued. In order to apply Theorem III.3.4 we must first choose S, S', $\underline{G}_{9} \underline{M}^{\prime}, \mu, \mu ', T, \underline{D}$, B, and $\underline{B}^{\prime}$ in such a way that $\mathrm{HI}$ - H8, $\mathrm{HII}-\mathrm{HI} 2, \mathrm{HCl}$, and $\mathrm{HC} 4$ are satisfied (see III.3.5). We set $\underline{S}=\underline{S}^{\prime}=X, \underline{M}=\underline{M}^{\prime}=\underline{B}=$ $=\underline{B}^{\prime}=\underline{N}, \mu=\mu$, and $\mu^{\prime}=v$. We define $T$ to be the identity mapping from $\underline{S}=X$ onto $\underline{S}^{\prime}=X$. Unfortunately we must assume that $\underline{D}$ can be chosen so that the hypotheses listed above are fulfilled; to carry out the discussion completely, we must also assume that $\mathrm{H}^{*}$ is satisfied. (The only natural choice for $\underline{D}$ in the abstract setting is $N$. It must be observed however that those hypotheses which require that $\underline{D}$ have certain countable subfamilies $\left(e^{\circ} \cdot g_{1}, H_{4}\right)$ are not likely to be fulfilled if $\left.\underline{D}=\underline{N}_{.}\right)$ Next, we define a non-negative function $w$ on 5 by $w S=1, s \in 5$. Define a non-negative function $W^{\prime}$ on $\underline{S}^{\prime} \times \underline{D}$ by

$$
W^{\prime}\left(s^{\prime}, D\right)=W_{*}^{\prime}\left(s^{\prime}, D, w\right), \quad\left(s^{\prime}, D\right) \in \underline{s^{\prime}} \times \underline{D}
$$

Evidently $W^{\prime}\left(s^{\prime}, D\right)=H_{D}^{\prime}\left(s^{\prime}\right),\left(s^{\prime}, D\right) \in \underline{S^{\prime}} X \underline{D}$. It is easy to check that $W$ ' is a weight function for $T$. Of course Hl3 is satisfied. As usual, let $W$ denote the weights induced by W'. Then

$$
W D=\int_{S^{\prime}} W^{\prime}\left(s^{\prime}, D\right) d \mu^{\prime}=\int_{X} H_{D^{\prime}}\left(s^{0}\right) d \nu=\nu D, \quad D \in D
$$

Hence $\mathrm{T}$ is BVW. By the Special Decomposition Theorem (III.3.4) there exists a strong Lebesgue-type decomposition $\left(W_{A}, W_{S}\right)$ for $W^{\prime}$ Then $W^{\prime}=W_{A}^{\prime}+W_{S}^{\prime}$, $T$ is $A_{A}$, and $W_{S}^{\prime}$ is strongly singular. For the corresponding weights we have $\nu \mid \underline{D}=W=W_{A}+W_{S}$. There 
exists a non-negative, $\mu$-integrable function $f_{A}$ defined on $S$ such that

$$
W_{A} D=\int_{D} f_{A}(B) d \mu, \quad D \in \underline{D}
$$

Moreover there is a set $B_{O}$ in $\underline{B}$ such that $\mu B_{0}=0$ and $W_{S^{\prime}}\left(s^{\prime}, \underline{S}\right)=0$ a.e. $\mu^{\prime}$ on $\mathrm{C}^{\cdot \mathrm{TB}} \mathrm{O}=\mathrm{CB}_{\mathrm{O}}$.

Define a non-negative function $\nu_{A}$ on $\underline{N}$ by $\nu_{A} M=\int_{M} f_{A}(s) d \mu$, $M \in N$. Then $\nu_{A}$ is a measure on $\underline{N}, \nu_{A}$ is $A C$ with respect to $\mu$, and $w_{A}=\nu_{A}$ ID. It is clear that $\nu_{A} D \leqq \nu D, D \in D$. Now let any set $M$ in $N$ be given. Then $M$ is in $M^{\prime}$ so that by $H 7^{*} \nu M=$ $=\inf \left[\mathrm{O}^{\prime}: 0^{\prime} \in \mathbb{D}^{\prime}, M \subseteq \mathrm{O}^{\prime}\right\}$. Since $T$ is the Identity mapping It is easily checked that $(1)$ consists of those subsets of $X$ which can be written as the union of a countable number of pairwise disjoint sets $D_{i}$ in $D_{\text {. Hence }}$

$$
\begin{aligned}
\nu M & =\inf \left\{\Sigma \nu D_{i}: M \subseteq U D_{i}, D_{i} \cap D_{k}=\varnothing \text { if } i \neq k\right\} \\
& \geqq \inf \left\{\Sigma \nu_{A} D_{i}: M \subseteq U D_{i}, D_{i} \cap D_{k}=\varnothing \text { if } i \neq k\right\} \\
& \geqq \nu_{A} M_{\bullet}
\end{aligned}
$$

Hence $\nu_{A} \leqq \nu$. We may therefore define a non-negative function $\nu_{S}$ on $\underline{M}$ by $\nu_{S} M=\nu M-\nu_{A} M, M \in \underline{N} \cdot \nu_{S}$ is a measure on $\underline{N}$ and $v_{S} I \underline{D}=w_{S} \cdot$ Finaliy, we assert that $\nu_{S} \mathrm{CB}_{0}=0$. To see this let $\varepsilon>0$ be given. Since $W_{S}^{\prime}\left(s^{\prime}, \underline{S}\right), s^{\prime} \in \underline{S}^{\prime}$, is $\mu^{\prime}$-integrable there is for every $t>0$ a number $\delta(t)>0$ such that $f_{M} \cdot W_{S}^{\prime}\left(s^{\prime}, \underline{S}\right) d \mu \mu^{\prime}<t$ 
if $M^{\prime}$ is in $\mu^{\prime}$ and $\mu^{\prime} M^{\prime}<\delta(t)$. By $H 7^{*}$ there is a set $O^{\prime}$ in (11) such that $\mathrm{CB}_{0} \subseteq 0^{\prime}$ and $\mu^{\prime} \mathrm{O}^{\prime}<\mu^{\prime} \mathrm{CB}_{\mathrm{O}}+\delta(\varepsilon)$. Hence $\mu^{\prime}\left(0 \cdot \cap \mathrm{B}_{0}\right)<\delta(\varepsilon)$. There is a countable number of pairwise disjoint sets $D_{i}$ in $\underline{D}$ such that $U D_{i}=0 \cdot \supseteq \mathrm{CB}_{0^{\circ}}$. It follows that $\nu_{S} C B_{0} \leqq \Sigma \nu_{S^{\prime}} D_{i}=\Sigma W_{S^{\prime}} D_{i}=\Sigma \int_{0}, W_{S}^{\prime}\left(s^{\prime}, D_{i}\right) d \mu^{\prime} \leqq \int_{0}, W_{S}^{\prime}\left(s^{\prime}, \underline{S}\right) d \mu \mu^{\prime}=$ $=\int_{0 \cdot \cap B_{0}} W_{S^{\prime}}\left(s^{\prime}, \underline{S}\right) d \mu^{\prime}+\int_{C_{B}} W_{S^{\prime}}\left(s^{\prime}, \underline{S}\right) d \mu^{\prime}<\varepsilon+0$. Hence $v_{s} \mathrm{CB}_{0}=0$

It should be noted that if $\underline{D}=\underline{N}$ the argument in the latter part of this discussion becomes much simpler. Moreover H7* is not required. 


\section{ON WEIGHT FUNCTIONS SATISFYING HI3}

Throughout this chapter we shall assume that $[\underline{S}, \underline{M}, \mu$ \} and $\left\{\underline{S}^{\prime}, \underline{M}^{\prime}, \mu^{\prime}\right.$ \} are measure spaces, that $T$ is a single valued transformation from $\underline{S}$ onto $\underline{S}^{\prime}$, that $\underline{D}$ is a family of subsets of

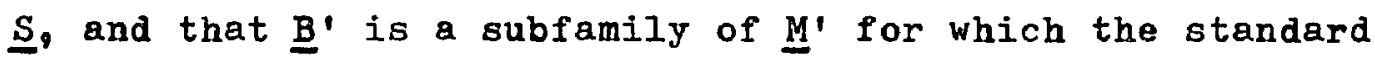
hypotheses $\mathrm{HI}-\mathrm{H} 8, \mathrm{HIl}$, and $\mathrm{Hl2}$ are satisfied. Thus, throughout this chapter, $\{\underline{S}, \underline{M}, \mu\},\left\{\underline{S}^{\prime}, \underline{M} \underline{M}^{\prime}, \mu^{\prime}\right\}, T, \underline{D}$, and $\underline{B}^{\prime}$ remain fixed and hypotheses $\mathrm{Hl}-\mathrm{H} 8, \mathrm{HIl}$, and $\mathrm{Hl} 2$ are always in force (unless it is specifically noted otherwịse). The remaining standard hypotheses will be assumed only when they are explicitly mentioned.

Certain notational conventions were set forth in Chapter $I$. We shall repeat several of them now. Thus 5 always denotes the family $\{S \subseteq S: S=N D, s \in D$, for some $s$ in $\underline{S}\}$. $\mathcal{E}$ always denotes the family of all subsets $E$ of $S$ each of which includes every $S$ in $S$ that it intersects. In addition, given $A \subseteq S, S_{A}$ always denotes the set $\{S \in S: S \cap A \not \varnothing\}$. Finally, if $w$ is a nonnegative function defined on $S$ then $W_{*}\left(s^{\prime}, D, w\right),\left(s^{\prime}, D\right) \in \underline{S}^{\prime} \times \underline{D}$, is always defined by

$$
w_{*}\left(s^{\prime}, D, w\right)=\Sigma W_{S}, \quad S \in \sigma_{s^{\prime}}, \quad S \subseteq D ; \quad\left(s^{\prime}, D\right) \in \underline{S}^{\prime} \times \underline{D} .
$$




\section{III.I Preliminary lemmas}

In this section we prove two simple but useful lemmas.

III.1.1. Lemma. Assume $w$ is a non-negative function defined on 5 . Define a function 0 on 5 by

$$
\hat{W}=\inf \left\{W_{*}(T S, D, W): S \subseteq D \in \underline{D}\right\}, \quad S \in S \text {. }
$$

1) Then $w S \leqq Q$ for every $S$ in 5 .

2) If $S_{0}$ is a set in $\dot{S}$ for which $\hat{w} S_{0}<\infty$ then $\hat{w} S_{0}=w S_{0}$.

Proof. First we prove I). Fix $S_{0}$ in 5. Suppose $\mathrm{D}_{0}$ is an

element of $\underline{D}$ for which $S_{0} \subseteq D_{0}$. Then $W_{*}\left(T S_{0}, D_{0}, w\right)=\Sigma w S$,

$\mathrm{S} \subseteq \mathrm{D}_{0} \cap \mathrm{T}^{-\mathrm{I}_{\mathrm{TS}}} \mathrm{O}^{\prime} \geqq \mathrm{wS}_{\mathrm{O}}$. Hence $\boldsymbol{\omega S}_{\mathrm{O}} \geqq \mathrm{wS}_{\mathrm{O}^{\circ}}$

Now we prove 2). Since $\hat{W S}_{0}<\infty$ there is a set $D^{*}$ in $\underline{D}$ such

that $S_{0} \subseteq D^{*}$ and $W_{*}\left(T S_{0}, D^{*}, W\right)<\infty^{\infty}$ Let $\varepsilon>0$ be given. There exist sets $S_{I}, \ldots, s_{m}$ in 5 such that the sets $s_{0}, s_{1}, \ldots, s_{m}$ are distinct, such that $s_{i} \subseteq D^{*}$ for $i=1, \ldots, m$, and such that

$\Sigma_{i=0}^{m} w S_{i}>W_{*}\left(T S_{0}, D^{*}, w\right)-\varepsilon$. By Lemma 8.4 of [2] there is a sequence of pairwise disjoint sets $D_{i}$ in $\underline{D}$ such that $S_{i} \subseteq D_{i} \subseteq D^{*}$ for $i=0,1, \ldots . m$. Then

$$
\begin{aligned}
W S_{0} & \leqq w_{*}\left(T S_{0}, D_{0}, w\right) \\
& \leqq w_{*}\left(T S_{0}, D^{*}, w\right)-\Sigma_{i=1}^{m} w_{*}\left(T S_{0}, D_{i}, w\right) \\
& <\Sigma_{i=0}^{m} w S_{i}+\varepsilon-\Sigma_{i=1}^{m} w S_{i} \\
& <w S_{0}+\varepsilon .
\end{aligned}
$$


Hence $\Delta S_{0}=w S_{0}$

Remark. Part 2) of Lemma III.1.1 is due to Reichelderfer.

III.1.2. Lemma. Assume $w$ is a non-negative function defined on 5. Again define a function $\hat{w}$ on 5 by

$$
\hat{w} S=\inf \left\{W_{*}(T S, D, W): S \subseteq D \in D\right\}, \quad S \in \sigma \text {. }
$$

Then the function $W:\left(s^{\prime}, D, w\right),\left(s^{\prime}, D\right) \in \underline{s^{\prime}} \times \underline{D}$, is also generated by

Proof. Using Lemma III.I.I we have $W_{*}^{\prime}\left(s^{\prime}, D, W\right) \leqq W !\left(s^{\prime}, D, 0\right)$, $\left(s^{\prime}, D\right) \in \underline{S}^{\prime} \times \underline{D}$. According to 8.5 of $[2], W_{*}^{\prime}\left(s^{\prime}, D, Q\right) \leqq W_{*}^{\prime}\left(s^{\prime}, D, w\right)$, $\left(s^{\prime}, D\right) \in \underline{S}^{\prime} \times \underline{D} . \quad$ (Actually Lemma 8.5 of [2] is not applicable here. In order to use it as it appears in [2] we must assume that

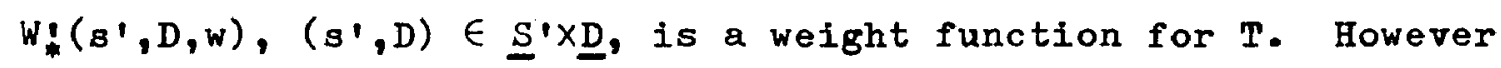
if one inspects the proof of 8.5 it becomes apparent that it requires only that $w_{\text {: }}$ be under additive. This requirement is fulfilled.)

\section{III.2 Measurability results}

III.2.1. Remark. Let $w$ be a non-negative function defined on 5. In view of 7.9 of $[2], w_{*}\left(s^{\prime}, D, w\right),\left(s^{\prime}, D\right) \in \underline{S^{\prime}} \times \underline{D}$, is a weight function for $T$ provided only that the function $W_{*}\left(s^{\prime}, D, w\right)$, $s^{\prime} \in \underline{S}^{\prime}$, is $\underline{M}^{\prime}-m e a s u r a b l e$ for every $D$ in $D$. There are no theorems in [2] - except 8.9 - that give conditions under which the function $W_{*}^{\prime}\left(s^{\prime}, D, w\right), s^{\prime} \in S^{\prime}$, is $M^{\prime}$-measurable for every $D$ in D. We shall now establish two such theorems, both of general interest. Theorem III.2.3 has as its forerunner the Theorem on page 217 of [1]. 
III.2.2. Definition. Let $w$ be a non-negative function defined on 5 . Define a non-negative function $g_{w}$ on $S$ by $B_{w}(s)=$ $=W S_{s}, s \in \underline{S}$, where $S_{s}$ is that member of 5 containing $s$. (Note that in view of Hll 5 partitions $\left.\underline{S}_{\cdot}\right)$ The function $g_{w}$ assumes a constant value on the subsets $S$ (in $\sigma$ ) of $\underline{S}$. It is called the point function induced by $w$ (or the induced point function).

III.2.3. Theorem. Assume HCl and HI5i. Let w be a nonnegative function defined on 5 . Suppose that the induced point function $g_{w}(s), s \in \underline{S}$, is $\underline{B}$-measurable.

I) Then the function $W_{*}^{\prime}\left(s^{\prime}, D, w\right), s^{\prime} \in \underline{S}^{\prime}$, is $M^{\prime}$-measurable for every $D$ in $\underline{D}$.

2) $W_{:}\left(s^{\prime}, D, w\right),\left(s^{\prime}, D\right) \in \underline{S}^{\prime} \times \underline{D}$, is a weight function for $T$. Proof. 2) follows from 1) and Remark 7.9 of [2]. It is sufficient to prove 1). The proof of 1 ) is carried out in three successively more general cases.

Case I. Assume $g_{w}$ is the characteristic function for some set $B$ in $\underline{B}$. Observe first that $B$ must also be in $\varepsilon$. Define $N\left(s^{\prime}, T, D \cap B\right)$ to be the number of sets $S$ in $S_{D \cap B}$ which are mapped onto $s^{\prime}$ by $T$. The function $N\left(s^{\prime}, T, D \cap B\right),\left(s^{\prime}, D\right) \in \underline{S}^{\prime} \times \underline{D}$, is rather similar to the crude multiplicity function defined in [I]. It is easy to verify that in the present case we have

$$
W_{*}^{\prime}\left(s^{\prime}, D, w\right)=N\left(s^{\prime}, T, D \cap B\right), \quad\left(s^{\prime}, D\right) \in \underline{S}^{\prime} \times \underline{D} \text {. }
$$

Our task now is to show that $N^{\prime}\left(B^{\prime}, T, D \cap B\right), s^{\prime} \in S^{\prime}$, is $M^{\prime}-$ measurable for every $D$ in $\underline{D}$. To do this we fix $D_{0}$ in $\underline{D}$. Define 
$\underline{D}^{* *}\left(D_{0}\right)$ to be the family of all finite subsets $\left\{D_{i}^{* *}\right\}_{i=1}^{n}$ of $\underline{D}^{* *}$ consisting of pairwise disjoint subsets of $D_{0}$; here, $\underline{D}^{* *}$ is the countable family whose existence is guaranteed by HI5i. The family $\underline{D}^{* *}\left(D_{0}\right)$ is countable. At this point we assert

$$
\begin{aligned}
N\left(s^{\prime}, T, D_{0} \cap B\right)= & \sup \left\{0, \sum_{i=1}^{n} H_{T\left(D_{1}^{* *} \cap B\right)}^{\prime}\left(s^{\prime}\right):\right. \\
& \left.\left(D_{i}^{* *}\right\}_{i=1}^{n} \in \underline{D}^{* *}\left(D_{0}\right)\right\},
\end{aligned}
$$

for every $s^{\prime}$ in $\underline{S}^{\prime}$

We prove (2). First assume that $\left\{D_{i}^{* *}\right\}_{i=1}^{n}$ is in $\underline{D}^{* *}\left(D_{0}\right)$. For each s' in $S^{\prime}$ we have

$$
\begin{aligned}
N\left(s^{\prime}, T ; D_{0} \cap B\right) & \geqq \sum_{i=I}^{n} N\left(s^{\prime}, T_{0}, D_{i}^{* *} \cap B\right) \\
& \geqq \sum_{i=1}^{n} H_{T\left(D_{i}^{* *} \cap B\right)}^{\prime}\left(s^{\prime}\right) .
\end{aligned}
$$

Hence

$$
\begin{aligned}
N\left(s^{\prime}, T, D_{O} \cap B\right) \geqq & \sup \left(0, \sum_{i=1}^{n} H_{T}^{\prime}\left(D_{i}^{* *} \cap B\right)\right. \\
& \left.\left\{D_{i}^{* *}\right\}_{i=1}^{n} \in \underline{s}^{\prime}\right):
\end{aligned}
$$

for every $s^{\prime}$ in $\underline{S}^{\prime}$.

Now fix $s^{\prime}$ in $\underline{S}^{\prime}$. If $N\left(s^{\prime}, T, D_{O} \cap B\right)=0$, then (2) follows from (3). Thus assume that $N\left(s^{\prime}, T, D_{O} \cap B\right)$ is positive. Let $m$ be any positive integer for which $N\left(s^{\prime}, T, D_{0} \cap B\right) \geqq m$. There 
exist distinct sets $S_{1}, \ldots, S_{m}$ in 5 such that $T S_{1}=s^{\prime}$ and $S_{1} \cong D_{0} \cap$ B. According to Lemma 8.4 of [2] there exist pairwise disfoint sets $D_{1}, \ldots, D_{m}$ in $\underline{D}$ such that $S_{i} \subseteq D_{i} \cong D_{0}$. By HI5i there exist sets $D_{i}^{* *}, \ldots, D_{m}^{* *}$ in $\underline{D}^{* *}$ such that $S_{i} \subseteq D_{i}^{* *} \subseteq D_{i}$ for $i=1, \ldots$, m. Evidently $\left[D_{i}^{* *}\right\}_{i=1}^{m}$ is in $\underline{D}^{* *}\left(D_{0}\right)$. Moreover $H_{T\left(D_{i}^{* *} \cap B\right)}\left(s^{\prime}\right)=1$. Hence $\Sigma_{i=1}^{m} H_{T}^{\prime}\left(D_{i}^{* * n B}\right)\left(s^{\prime}\right)=m$. It follows that sup $\left\{0, \Sigma_{i=1}^{n} H_{T}^{\prime}\left(D_{i}^{* *} \cap B\right)\left(s^{\prime}\right):\left\{D_{i}^{* *}\right\}_{i=1}^{n} \in D^{* *}\left(D_{0}\right)\right\} \geqq N\left(s^{\prime}, T, D_{0} \cap B\right)$. Hence (2) holds in this case also. Thus (2) holds for every $s^{\prime}$

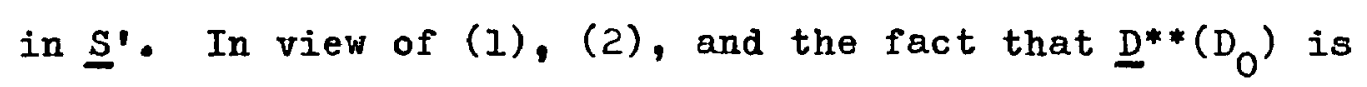
countable, it is now apparent that it suffices to show that each function $H_{T}^{\prime}\left(D_{i}^{* * \cap B}\right)\left(s^{\prime}\right), s^{\prime} \in \underline{S}^{\prime}$, is $\underline{M}^{\prime}-$ measurable. But by HCl each set $T\left(D_{i}^{* *} \cap B\right)$ is in $M^{\prime}$ and so $H_{T}^{\prime}\left(D_{i}^{* *} \cap B\right)$ is $\underline{M}^{\prime}$-measurable. Case II. Assume that $g_{w}$ is bounded. Thus we assume that there is a positive number $t$ for which $g_{W}(s) \leqq t, s \in \underline{S}$. We shall use the fact that 1) holds for Case I to prove it for the more general Case II.

Let $m$ be a fixed positive integer. For each $j=0,1, \ldots$, $2^{m}$ we define

$$
B_{m j}=\left\{s \in \underline{S}: \frac{j t}{2^{m}} \leqq B_{w}(s)<\frac{(j+1) t}{2^{m}}\right\} .
$$

Then $B_{m j}$ is in $\underline{B}$ for each $j$. The characteristic function $H_{m j}(s)$, 
$s \in \underline{S}$, of the subset $B_{m j}$ of $S$ is $B$-measurable. Each $B_{m j}$ is also in $\mathcal{E}$ and so, given $S$ in $5, H_{m j} S$ consists of but one number $r_{m j}(S)$. Define a non-negative function $w_{m j}$ on 5 by $w_{m j} s=r_{m j}(s), s \in S$. Surely $B_{w_{m j}}=H_{m j}$. Thus we may apply the result of Case $I$ to conclude that the function $W_{*}^{\prime}\left(s^{\prime}, D, w_{m j}\right), s^{\prime} \in \underline{S}^{\prime}$, is $\underline{M}^{\prime}$-measurable for every $D$ in $D$. We define a non-negative function $w_{m}$ on 5 by

$$
w_{m} s=\sum_{j=0}^{2^{m}} \frac{j t}{2^{m}} w_{m j} s, \quad s \in \sigma .
$$

By Lemma I.7.9 we have

$$
w_{*}\left(s^{\prime}, D, w_{m}\right)=\sum_{j=0}^{2^{m}} \frac{j t}{2^{m}} w_{*}^{\prime}\left(s^{\prime}, D, w_{m j}\right), \quad\left(s^{\prime}, D\right) \in \underline{s}^{\prime} \times \underline{D} .
$$

It follows that the function $W_{*}^{\prime}\left(s^{\prime}, D, w_{m}\right), s^{\prime} \in S^{\prime}$, is $M^{\prime}-$ measurable for every $D$ in $D$ and for every positive integer $m$. For each $m \geqq 1$ the set $S$ is the union of the pairwise disjoint sets $B_{m j}, j=0,1, \ldots, 2^{m}$. And $B_{m j}=B_{m+1,2 j} \cup B_{m+1,2 j+1}$ for every $m$ and $j$. It follows that $w_{m} \leqq w_{m+1}, m \geqq l$, and $0 \leqq w S-w_{m} S<\frac{t}{2^{m}}, S \in S$. Hence $\left\{w_{m}\right\}_{m=I}^{\infty}$ is a non-decreasing sequence of functions and $w S=I i m_{m} w_{m} S, S \in S$. By Lemma I.7.10

$$
w_{*}^{\prime}\left(s^{\prime}, D, w\right)=1 i m_{m} w_{*}^{\prime}\left(s^{\prime}, D, w_{m}\right), \quad\left(s^{\prime}, D\right) \in \underline{s} \cdot \times \underline{D} .
$$

Now fix $D$ in $D$. We have already observed that each function $w_{*}\left(s^{\prime}, D, w_{m}\right), \quad s^{\prime} \in \underline{s}^{\prime}$, is $M^{\prime}$-measurable. It follows from 
(4) that $W_{:}^{\prime}\left(s^{\prime}, D, w\right), s^{\prime} \in \underline{S}^{\prime}$, must also be $\underline{M}^{\prime}$-measurable. We now know that 1) holds in Cases I and II. We shall use these facts to show that 1) holds in every case. Thus suppose $\mathrm{B}_{\mathrm{W}}$ is an arbitrary non-negative, B-measurable function. Define a sequence $\left\{w_{m}\right\}_{m=1}^{\infty}$ of functions on 5 by $w_{m} S=\min (w S, m), s \in 5$. For each $m \geqq 1, W_{*}^{\prime}\left(s^{\prime}, D, w_{m}\right), s^{\prime} \in S^{\prime}$, is $\underline{M}^{\prime}$-measurable for every $D$ in D; this follows from the result of Case II. Since wS $=$ $=I i m_{m} w_{m} S, S \in S$, and since $w_{m} \leqq w_{m+1}, m \geqq 1$, it follows from Lemma I.7.10 that

$$
\lim _{m} W_{*}\left(s^{\prime}, D, w_{m}\right)=W_{*}^{\prime}\left(s^{\prime}, D, w\right), s^{\prime} \in \underline{S}^{\prime},
$$

for every $D$ in $D$. Hence $W_{*}^{\prime}\left(s^{\prime}, D, w\right), s^{\prime} \in S^{\prime}$, is $M^{\prime}$-measurable for every $D$ in $D$. The proof of 1 ) is complete.

III.2.4. Theorem. Assume H9, H15i, and HCl. Define a non-negative function $a$ on 5 by

$$
\text { QS }=\inf (W \cdot(T S, D): S \subseteq D \in \underline{D}\}, \quad S \in S \text {. }
$$

1) There exists a non-negative function w defined on 5 such that the Induced point function $\mathrm{g}_{w}(s), s \in \underline{S}$, is $\underline{B}$-measurable and for which there exists a set $B^{\prime}$ in $\underline{B}^{\prime}$ such that $\mu^{\prime} B^{\prime}=0$ and $g_{W}(s)=g_{Q}(s), s \in C^{-1} B^{\prime}$.

2) For every $D$ in $\underline{D}$ the function $W_{*}\left(s^{\prime}, D, \hat{w}\right), s^{\prime} \in \underline{S}^{\prime}$, is M'-measurable.

3) $W_{*}^{\prime}\left(B^{\prime}, D, \hat{W}\right),\left(B^{\prime}, D\right) \in \underline{S}^{\prime} \times \underline{D}$, is a weight function for $T$. Proof. Note first that $W \cdot\left(T S, D^{* *}\right) \leqq W^{\prime}(T S, D)$ whenever 
$S$ is in $\Theta$ and $D^{* *} \subseteq D$. Thus it follows from HI5i that

$$
\hat{w} S=\inf \left\{W^{\prime}\left(T S, D^{* *}\right): S \subseteq D^{* *} \in \underline{D}^{* *}\right\}, \quad S \in \mathbf{5}
$$

We first prove I). By 2.12 of [2] (see I.4) there 1s, for every $D$ in $D$, a non-negative function $h_{D}^{\prime}$ on $\underline{S}^{\prime}$ which is $\underline{B}^{\prime}$-measurable and for which we have $h_{D^{\prime}}\left(s^{\prime}\right)=W^{\prime}\left(s^{\prime}, D\right)$ a.e. $\mu^{\prime}$ on $\underline{s}^{\prime} \cdot$ Define $X_{D}^{\prime}=\left\{s^{\prime} \in \underline{S}^{\prime}: h_{D}^{\prime}\left(s^{\prime}\right) \neq W^{\prime}\left(s^{\prime}, D\right)\right\}$. Then $X_{D}^{\prime}$ is in $\underline{M}^{\prime}$ and $\mu^{\prime} X_{D}^{\prime}=0$. By $H 6$ there is for every $D$ a set $B_{D}^{\prime}$ in $\underline{B}^{\prime}$ such that $X_{D}^{\prime} \subseteq B_{D}^{\prime}$ and $\mu^{\prime} B_{D}^{\prime}=0$. Now we define $B^{\prime}=U B_{D^{* *}}^{\prime}, D^{* *} \in \underline{D}^{* *}$. $\underline{D}^{* *}$ is countable and each $B_{D^{* *}}$ is in $\underline{B}^{\prime}$. Hence $B^{\prime}$ is in $\underline{B}^{\prime}$, and $\mu^{\prime} B^{\prime}=0$. Moreover

$$
h_{D^{* *}}^{\prime}\left(s^{\prime}\right)=W^{\prime}\left(s^{\prime}, D^{* *}\right), \quad s^{\prime} \in C^{\prime} B^{\prime}, D^{* *} \in \underline{D}^{* *}
$$

For each $D^{* *}$ in $D^{* *}$ we define a non-negative function $t_{D^{*}}$ on $S$ by $t_{D^{* *}}(s)=h_{D^{* *}}(T s), s \in \underline{S}$. Jsing the fact that each $h_{D}^{\prime}$ is $\underline{B}^{\prime}$-measurable and the fact that $T^{-1} \underline{B}^{\prime} \subseteq \underline{B}$ we can prove easily that the functions $t_{D^{* *}}(s), s \in \underline{S}$, are B-measurable. Observe that

$$
t_{D^{* *}}(s)=W^{\prime}\left(T s, D^{* *}\right), \quad s \in \mathrm{CT}^{-1} B^{\prime}, D^{* *} \in \underline{D}^{* *}
$$

Each function $t_{D^{* *}}$ is constant on the sets $S$ in 5 . Hence for each $S$ in $S$ the set $t_{D^{*} *} S$ consists either of a single real number or just of $\infty$. Let $p\left(D^{* *}, S\right)$ be the unique element of the set $t_{D^{* *}}$ s. We define non-negative functions $w_{D^{*}}$ on 5 by 


$$
w_{D^{* *}} S= \begin{cases}p\left(D^{* *}, S\right) & \text { if } S \subseteq D^{* *} \\ p(\underline{S}, S) & \text { if } S \cap D^{* *}=\varnothing\end{cases}
$$

For the induced point functions we have

$$
g_{w_{D^{* *}}}(s)= \begin{cases}t_{D^{* *}}(s), & \text { if } s \in D^{* *} \\ t_{\underline{S}}(s), & \text { if } s \notin D^{* *}\end{cases}
$$

Each function $\mathrm{g}_{\mathrm{w}_{\mathrm{D}^{*}}}(\mathrm{~s}), \mathrm{s} \in \underline{\mathrm{S}}$, is B-measurable. Finally we can introduce the desired function $w$. We define a non-negative function $w$ on $S$ by $w S=\inf \left\{w_{D^{* *}} S: D^{* *} \in \underline{D}^{* *}\right\}, S \in \mathcal{S}$. Surely $g_{w}(s)=\inf \left\{B_{W^{*}}(s): D^{* *} \in \underline{D}^{* *}\right\}, s \in \underline{S} \cdot$ The function $B_{W}(s)$, s $\in \underline{S}$, is evidently $\underline{B}$-measurable. We now assert

$$
w S=\hat{H} S, \quad S \subseteq C T^{-1} B^{\prime}
$$

(Observe that it follows from Hl2 that either $S \subseteq T^{-1} B^{\prime}$ or $S \subseteq C T^{-1} B^{\prime}$ whenever $S$ is in $\left.S_{.}\right)$

To see (4) we assume $S_{0} \subseteq C T^{-1} B^{\prime}$. Let $D^{* *}$ be any element of

$D^{* *}$ for which $S_{O} \subseteq D^{* *}$. Using (3) we obtain

$$
w \cdot\left(T S_{O}, D^{* *}\right)=t_{D^{* *}} S_{O}=w_{D *} S_{O} \geqq w S_{O} \cdot
$$

In view of (1)

$$
\text { คS }_{0} \geqq \text { WS }_{0}
$$

Now suppose that $D^{* *}$ is any set in $\underline{D}^{* *}$. If $S_{0} \subseteq D^{* *}$ then $w_{D^{* *}} S_{O}=t_{D^{* *}} S_{O}=w^{\prime}\left(T S_{O}, D^{* *}\right) \geqq A S_{O} ;$ but if $S_{O} \cap D^{* *}=\varnothing$ then 
$w_{D^{* *}} S_{0}=t_{S^{S}} S_{0}=W^{\prime}\left(T S_{0}, \underline{S}\right) \geqq A S_{0}$. In either event $w_{D^{* *}} S_{0} \geqq A S_{0}$ ConsequentIy

$$
w S_{0} \geqq A S_{0}
$$

From (5) and (6) we obtain $w_{O}=0 S_{O}$. Thus (4) is proved.

It follows from (4) that $g_{W}(s)=g_{\hat{W}}(s), s \in \mathrm{CT}^{-1} \mathrm{~B}^{\prime}$. It is now clear that the function $w$ fulfills the requirements of 1 ). Using this same function w together with (4) we shall now establish the truth of 2).

Thus fix $D$ in $D$. Suppose $s^{\prime}$ is in $C^{\prime} B^{\prime}$. Then $T^{-1} s^{\prime} \cap T^{-1} B^{\prime}=$ $=\varnothing$ so that if $S$ is in $S_{S^{\prime}}$, it is true that $S \subseteq \mathrm{CT}^{-1} \mathrm{~B}^{\prime}$. It follows easily from (4) that $W_{*}^{\prime}\left(s^{\prime}, D, W^{\prime}\right)=W_{*}\left(s^{\prime}, D, \hat{D}\right)$. Since $\mu^{\prime} B^{\prime}=0$ we have evidently shown that $w_{*}^{\prime}\left(s^{\prime}, D, w\right)=w_{*}^{\prime}\left(s^{\prime}, D, \omega\right)$ a.e. $\mu^{\prime}$ on $\underline{S}^{\prime}$ uniformly with respect to $\underline{D}$. By Theorem III.2.3 the function $W_{*}^{\prime}\left(s^{\prime}, D, w\right), s^{\prime} \in \underline{S}^{\prime}$, is $M^{\prime}$-measurable for every $D$ in $D$. The measure space $\left\{\underline{S}^{\prime}, \underline{M}^{\prime}, \mu^{\prime}\right\}$ is complete and so the function $W_{*}^{\prime}\left(s^{\prime}, D, \hat{D}\right), s^{\prime} \in \underline{S}^{\prime}$, must be $\underline{M}^{\prime}$-measurable for every $D$ in $\underline{D}$. Thus 2) is proved. 3) follows from 2) and Remark 7.9 of [2].

\section{III.2.5. Corollary. Assume HI5i and HCI. Assume that W'} is a weight function for T which satisfies HI3. Then there exists a non-negative function $w_{0}$ defined on 5 such that the induced point function $B_{w_{0}}(s), s \in \underline{S}$, is $\underline{B}$-measurable, such that $w_{*}^{\prime}\left(s^{\prime}, D, w_{0}\right) \leqq w^{\prime}\left(s^{\prime}, D\right),\left(s^{\prime}, D\right) \in \underline{S}^{\prime} \times \underline{D}$, and such that $w_{*}^{\prime}\left(s^{\prime}, D, w_{0}\right)=$ $=W^{\prime}\left(s^{\prime}, D\right)$ a.e. $\mu^{\prime}$ on $\underline{S}^{\prime}$ uniformly with respect to $\underline{D}$. 
Proof. We define a non-negative function on 5 by

$$
\text { WS }=\inf \{W \cdot(T S, D): S \subseteq D \in \underline{D}\}, \quad S \in \mathcal{E} \text {. }
$$

By Lemma III.1.2 the function $W$ is generated by $\omega$. By. Theorem III.2.4 there exists a non-negative function $w_{\text {\# }}$ defined on 5 such that the induced point function $\mathrm{B}_{\mathrm{w}_{\#}}(\mathrm{~s}), \mathrm{s} \in \underline{\mathrm{S}}$, is $\underline{\mathrm{B}}-$ measurable and for which there exists a set $B^{\prime}$ in B $^{\prime}$ such that $\mu^{\prime} B^{\prime}=0$ and

$$
\mathbf{B}_{\mathbf{w}_{H}}(s)=\mathbf{B}_{\boldsymbol{Q}}(\mathbf{s}), \quad s \in C \mathrm{~T}^{-1} \mathbf{B}^{\prime}
$$

(1) is equivalent to

$$
w_{\#} S=A S, \quad S \subseteq C T^{-1} B^{\prime}
$$

Define a non-negative function $w_{0}$ on 5 by

$$
w_{0} S= \begin{cases}w_{\#} S \text { if } S \subseteq C T^{-I} B^{\prime} \\ 0 \text { if } S \cap C T^{-I_{B^{\prime}}=\varnothing}\end{cases}
$$

Surely $g_{w_{0}}(s), s \in \underline{S}$, is $\underline{B}$-measurable. By $(2) w_{*}^{\prime}\left(s^{\prime}, D, w_{0}\right) \leqq$ $\leqq W^{\prime}\left(s^{\prime}, D\right),\left(s^{\prime}, D\right) \in \underline{s}^{\prime} \times \underline{D}$. Jsing the fact that $w_{0} S=$ as whenever $S \subseteq \mathrm{CT}^{-1} \mathrm{~B}^{\prime}$ it follows readily that

$$
W_{*}\left(s^{\prime}, D, w_{0}\right)=W^{\prime}\left(s^{\prime}, D\right), \quad s^{\prime} \in C^{\prime} B^{\prime}, \quad D \in \underline{D} .
$$

The proof is complete since $\mu^{\prime} B^{\prime}=0$.

III.2.6. Remark. It follows from Corollary III.2.5 that every weight function satisfying Hl3 is effectively generated by some function $w$ on $\sigma$ whose induced point function is B-measurable. 
This result will be used several times in the sequel. It appears to be a significant tool in the study of those weight functions which satisfy HI3.

III.3 The Special Decomposition Theorems

III.3.1. Remarks. According to 7.8 of [2], every weight function satisfying $\mathrm{Hl} 3$ is over additive and hence almost over additive. Evidently the results of Section II.5 can be applied directly to obtain decomposition theorems for weight functions of this special type. We shall adopt this approach. However it is also possible to obtain the desired result from the General Decomposition Theorem (Theorem II.2.8). The latter approach requires lengthier proofs but fewer of the standard hypotheses. We shall present an outline of the second approach, giving few details.

\section{III.3.2. Iemma. Assume $W_{0}^{\prime}$ is a weight function for $T$} which satisfies H13. Suppose that $W_{1}$ and $W_{2}^{\prime}$ are weight functions for $T$ for which $w_{0}^{\prime}=w_{1}^{\prime}+w_{2}^{\prime}$. Define, for $i=0,1,2$, a nonnegative function $\theta_{i}$ on $S$ by

$$
\hat{w}_{i} S=\inf \left\{w_{i}(T S, D): S \subseteq D \in \underline{D}\right\}, \quad S \in S \text {. }
$$

Then

$$
\hat{\omega}_{0} s=\omega_{1} s+\omega_{2} s, \quad s \in E,
$$

and

$$
w_{0}^{\prime}\left(s^{\prime}, D\right)=w_{*}^{\prime}\left(s^{\prime}, D, \hat{O}_{1}\right)+w_{*}\left(s^{\prime}, D, \hat{w}_{2}\right), \quad\left(s^{\prime}, D\right) \in \underline{S}^{\prime} \times \underline{D} .
$$


Moreover if $\left(s_{0}^{\prime}, D_{0}\right)$ is an element of $\underline{S} \times \underline{D}$ for which $w_{0}\left(s_{0}^{\prime}, D_{0}\right)<\infty$ then $W_{i}\left(s_{0}^{\prime}, D_{0}\right)=W_{1}\left(s_{0}^{\prime}, D_{0}, \hat{Q}_{1}\right), i=1,2$. Hence if $T$ is $B W_{0}$ then $w_{i}^{\prime}\left(s^{\prime}, D\right)=W_{*}^{\prime}\left(s^{\prime}, D, \hat{W}_{i}\right)$ a.e. $\mu^{\prime}$ on $\underline{S}^{\prime}$ uniformly with respect to $\underline{D}$.

Proof. We prove (1) first. We assert that

$$
\hat{\theta}_{1} s+\theta_{2} s \leqq \theta_{0} s, \quad s \in S \text {. }
$$

Surely (3) holds in case $\theta_{0} S=\infty$. Thus assume $\hat{Q}_{0} S<\infty$ and let $p$ be a positive real number for which $\omega_{0} s<p$. There exists a set $D_{p}$ in $\underline{D}$ such that $S \subseteq D_{p}$ and $W_{o}\left(T S, D_{p}\right)<p$. It follows that $a_{1} S+a_{2} S \leqq w_{i}\left(T S, D_{p}\right)+w_{2}\left(T S, D_{p}\right)=w_{0}\left(T S, D_{p}\right)<p$. Hence (3) also holds in case $\theta_{0} S<\infty$. Next we prove

$$
\hat{w}_{0} s \leqq \hat{\omega}_{1} s+\hat{a}_{2} s, \quad s \in \epsilon .
$$

Surely (4) holds in case $\hat{w}_{1} s+\hat{\theta}_{2} s=\infty$. Thus assume $\hat{w}_{1} S+\hat{w}_{2} s<\infty$ and suppose $\hat{w}_{1} S+\hat{O}_{2} S<p$. There exist sets $D_{1}$ and $D_{2}$ in $\underline{D}$ such that $S \subseteq D_{1}, S \subseteq D_{2}$, and $w_{1}\left(T S, D_{1}\right)+w_{2}^{\prime}\left(T S, D_{2}\right)<p$. By Lemma 8.3 of [2] there is a set $D_{p}$ in $\underline{D}$ such that $S \subseteq D_{p} \cong D_{1} \cap D_{2}$. Using the under additivity of the $W_{i}$ 's we obtain

$$
\begin{aligned}
Q_{0} S & \leqq w_{0}\left(T S, D_{p}\right) \\
& \leqq w_{i}\left(T S, D_{p}\right)+w_{2}\left(T S, D_{p}\right) \\
& \leqq w_{i}\left(T S, D_{1}\right)+w_{2}\left(T S, D_{2}\right)<p .
\end{aligned}
$$

Hence (4) holds in case $\theta_{1} s+\theta_{2} s<\infty$. (1) now follows from (3) and (4). (2) follows from (1), Lemma I.7.9, and Lemma III.1.2. 
Assume now that $\left({ }_{0}, D_{0}\right)$ is an element of $\underline{S} \times \underline{D}$ for which

$w_{0}\left(s_{0}^{\prime}, D_{0}\right)<\infty$. In view of (2) we have

(5)

$$
w_{0}^{\prime}\left(s_{0}^{\prime}, D_{0}\right)=w_{*}^{\prime}\left(s_{0}^{\prime}, D_{0}, A_{1}\right)+w_{*}^{\prime}\left(s_{0}^{\prime}, D_{0}, \hat{W}_{2}\right)
$$

and, by hypothesis,

$$
W_{0}^{\prime}\left(s_{0}^{\prime}, D_{0}\right)=W_{1}^{\prime}\left(s_{0}^{\prime}, D_{0}\right)+W_{2}^{\prime}\left(s_{0}, D_{0}\right) \text {. }
$$

Since $w_{0}\left({ }^{j} \dot{0}, D_{0}\right)<\infty$ we can deduce from (5) and (6) that $w_{i}\left(s_{0}, D_{0}\right)$ and $W_{:}\left(s_{0}, D_{0}, \hat{W}_{i}\right)$ are finite $(i=1,2)$ and finally that

$$
0=\left(w_{1}^{\prime}\left(s_{0}^{\prime}, D_{0}\right)-w_{*}^{\prime}\left(s_{0}^{\prime}, D_{0}, \hat{w}_{1}\right)\right)+\left(w_{2}^{\prime}\left(s_{0}^{\prime}, D_{0}\right)-w_{*}^{\prime}\left(s_{0}^{\prime}, D_{0}, \theta_{2}\right)\right)
$$

But, according to Lemma 8.5 of [2], we must have

$$
W_{i}^{\prime}\left(s_{0}^{\prime}, D_{0}\right)-W_{*}^{\prime}\left(s_{0}^{\prime}, D_{0}, \hat{\omega}_{i}\right) \geqq 0, i=1,2 \text {. }
$$

It follows from (7) and (8) that $w_{i}\left(s_{0}, D_{0}\right)=w_{*}\left(s_{0}, D_{0}, \hat{w}_{i}\right)$, $i=1,2$.

III.3.3. The following theorem may seem out of place in this section. However it is a result of independent interest.

Theorem. Assume HC4. Assume $W^{\prime}$ is a weight function for T which satisfies HI3. Assume that $T$ is BVW. Assume that $W^{\prime}$ is an AC part of W'. Then $W_{a}$ essentially satisfies HI3 in the following sense: There exists a non-negative function wo defined on $S$ such that $W_{*}^{\prime}\left(s^{\prime}, D, w_{0}\right) \leqq W_{a}^{\prime}\left(s^{\prime}, D\right),\left(s^{\prime}, D\right) \in \underline{S} \times \underline{D}$, and such that $W_{*}^{\prime}\left(s^{\prime}, D, W_{0}\right)=W_{a}^{\prime}\left(s^{\prime}, D\right)$ a.e. $\mu^{\prime}$ on $\underline{S}^{\prime}$ uniformly with respect to $\underline{D}$.

Proof. By Theorem II.I.14 there exist weight functions W' 
and $W_{\#}$ for $T$ such that $W^{\prime}=W_{A}^{\prime}+W_{\#}^{\prime}, W_{A}^{\prime} \leqq W_{Q}^{\prime}, T$ is $A C W_{A}$, and such that $W_{A}^{\prime}=W_{a}^{\prime}$ a.e. $\mu^{\prime}$ on $\underline{S}^{\prime}$ uniformly with respect to $\underline{D}$. We define a non-negative function $w_{0}$ on 5 by $w_{0} S=\operatorname{lnf}\left[W_{A}(T S, D):\right.$ $S \subseteq D \in \underline{D}\}, S \in E$. Since $T$ is BVW it follows from Lemma III.3.2 that $W_{A}^{\prime}\left(s^{\prime}, D\right)=W_{*}^{\prime}\left(s^{\prime}, D, W_{0}\right)$ a. $\theta_{0} \mu^{\prime}$ on $\underline{S}^{\prime}$ uniformly with respect to D. Moreover Lemma 8.5 of [2] guarantees that $w_{*}\left(s^{\prime}, D, w_{0}\right) \leqq$ $W_{A}^{\prime}\left(s^{\prime}, D\right),\left(s^{\prime}, D\right) \in \underline{S}^{\prime} \times \underline{D}$. It now follows at once that $w_{0}$ has the desired properties.

III.3.4. The special decomposition theorem. Assume HI3, H4*, H7*, HCl, and HC2. Assume that $\mathrm{T}$ is BVW.

1) Then every Lebesgue-type decomposition for W' is a strong Lebesgue-type decomposition for 'W'. The components of such a decomposition must essentially satisfy HI3 (see III.3.3) and hence are almost over additive.

2) Assume also $\mathrm{HC} 4$. Then there exists a strong Lebesguetype decomposition $\left(W_{a}^{\prime}, W_{B}\right)$ for $W^{\prime}$. Its components satisfy HI3. Proof. By Lemma 7.8 of [2] W' is (almost) over additive. 1). follows at once from Theorem II.5.2 and Lemma III.3.2. We now prove 2). Applying the Strong Decomposition Theorem (Corollary II.5.3) we deduce that there exists a strong Lebesguetype decomposition $\left(W_{A}, W_{\sharp}\right)$ for $W^{\prime}$. Thus, among other things, $W^{\prime}=W_{A}^{\prime}+W_{\sharp} \cdot$ By Lemma III.3.2 there exist non-negative functions $w_{a}$ and $w_{s}$ defined on $S$ for which 


$$
W^{\prime}\left(s^{\prime}, D\right)=W_{*}\left(s^{\prime}, D, w_{a}\right)+W_{*}\left(s^{\prime}, D, w_{s}\right), \quad\left(s^{\prime}, D\right) \in \underline{S}^{\prime} \times \underline{D} ;
$$

moreover, $W_{A}^{\prime}\left(s^{\prime}, D\right)=W_{*}^{\prime}\left(s^{\prime}, D, W_{Q}\right) \quad a \cdot \theta \cdot \mu^{\prime}$ on $\underline{S}^{\prime}$ uniformly with respect to $D$ and $W_{\#}^{\prime}\left(s^{\prime}, D\right)=W_{*}\left(s^{\prime}, D, W_{s}\right)$ a.e. $\mu^{\prime}$ on $\underline{S}^{\prime}$ uniformly with respect to $\underline{D}$. We define non-negative functions $W_{a}$ and $W_{s}$ on $\underline{S} \times \underline{D}$ by

$$
w_{a}^{\prime}\left(s^{\prime}, D\right)=W_{*}^{\prime}\left(s^{\prime}, D, w_{a}\right), \quad\left(s^{\prime}, D\right) \in \underline{S} \cdot \underline{D}
$$

and

$$
W_{s}^{\prime}\left(s^{\prime}, D\right)=W_{*}^{\prime}\left(s^{\prime}, D, w_{s}\right), \quad\left(s^{\prime}, D\right) \in \underline{S^{\prime}} \times \underline{D} .
$$

Using H2, Remark 7.9 of [2], and the fact that $W_{A}^{\prime}$ and $W_{\#}^{\prime}$ are weight functions for $T$, we deduce that $W_{a}^{\prime}$ and $W_{b}^{\prime}$ are weight functions for $T$. It follows from (1) and Lemma $I .7 .5$ that $\left(W_{Q}, W_{s}^{\prime}\right)$ is a strong Lebesgue-type decomposition for W'. By construction the components $W_{a}^{\prime}$ and $W_{s}^{\prime}$ satisfy HI.3.

III.3.5. Remarks. It was stated in III.3.I that the results of Theorem III.3.4 can be derived from the General Decomposition Theorem. We shall describe briefly the arguments used. Most details are omitted. Several preliminary results are needed.

i) Assume HCl. Assume that $W^{\prime}$ is a weight function for $\mathbf{T}$ generated by $w$. Suppose that the induced point function $g_{w}$ is B-measurable. Define $S^{+}=U S, S \in S$ and $w S>0$. Assume that $T$ is BVW. Then $T$ is $A C W$ if and only if for every set $B$ in $B \cap E$ for which $B \leqq S^{+}$and $\mu B=0$ it is true that $\mu \cdot T B=0$. 
The straightforward proof rests chiefly upon Theorem 7.24 of [2]. Lemma I.7.8 is also used at one point.

ii) Assume $\mathrm{HCl}$ and Hl5i. Suppose W' is a weight function for $T$ satisfying HI3. Assume that $P$ is $B V W$ and that $W '$ is singular. Then $W^{\prime}$ is strongly singular.

We sketch a proof for ii). In view of Corollary III.2.5 and Lemma I.7.5 it is sufficient to prove ii) for weight functions W' generated by functions $w$ whose induced point functions $g_{w}$ are B-measurable. We write $\underline{S}^{\prime}$ as the union of a countable number of pairwise disjoint sets $M_{n}^{\prime}, n \geqq I$, in $M^{\prime}$ of finite $\mu^{\prime}$ measure. Define $S^{+}=U S, S \in S$ and $w S>0$. Define $\Gamma=(B \in \underline{B} \cap \varepsilon$ : $B \subseteq S^{+}$and $\left.\mu B=0\right\}$ and $\Gamma_{n}^{\prime}=\left\{M^{\prime} \in \underline{M}^{\prime}: M^{\prime}=M_{n}^{\prime} \cap T B\right.$ for some $B$ in $\Gamma$, $n \geqq 1 . \quad$ Set $p_{n}=\sup \left[\mu^{\prime} M^{\prime}: M^{\prime} \in \Gamma_{n}^{\prime}\right\}, n \geqq 1$. Using an argument rather similar to the one used early in the proof of Lemma II.5.I we obtain a set $B_{0}$ in $\underline{B} \cap \varepsilon$ such that $\mu \cdot\left(M_{n}^{\prime} \cap T B_{0}\right)=p_{n}, n \geqq 1$. The set ${ }^{T B}{ }_{0}$ is in $\underline{M}^{\prime}$. There exists a set $B_{0}^{\prime}$ in $\underline{B}^{\prime}$ such that $\mathrm{TB}_{\mathrm{O}} \subseteq \mathrm{B}_{0}^{\prime}$ and $\mu^{\prime}\left(\mathrm{B}_{0}^{\prime} \cap \mathrm{C}^{\prime T B_{0}}\right)=0$. The set $\mathrm{S}^{+} \cap \mathrm{CT}^{-1} \mathrm{~B}_{0}^{\prime}$ is in $\underline{B} \cap \varepsilon$. Define a non-negative function $w_{1}$ on $S$ by

$$
w_{1} S=\left\{\begin{array}{l}
w S, \quad \text { if } S \subseteq S^{+} \cap \mathrm{CT}^{-1} \mathrm{~B}_{0}^{\prime} \\
0, \quad \text { if } S \cap\left(S^{+} \cap \mathrm{CT}^{-1} \mathrm{~B}_{0}^{\prime}\right)=\varnothing
\end{array}\right.
$$

Using Theorem III.2.3 and $i)$ we prove that $W_{*}^{\prime}\left(s^{\prime}, D, w_{1}\right),\left(s^{\prime}, D\right) \epsilon$ $\in \underline{S}^{\prime} \times \underline{D}$, is an AC part of $W^{\prime} \cdot$ Hence $W_{*}^{\prime}\left(s^{\prime}, \underline{S}, w_{1}\right)=0$ a.e. $\mu^{\prime}$ 
on $\underline{S}^{\prime}$. Using 7.7 of [2] it is now easy to see that $W^{\prime}\left(s^{\prime}, \underline{S}\right)=0$ a.e. $\mu$ ' on $\mathrm{C}^{\prime} \mathrm{TB}_{0}$.

iii) Assume HI5i, HCI, and HC4. Assume that W' is a weight function for $T$ satisfying $H 13$. Assume that $T$ is BVW.

1) Then every Lebesgue-type decomposition for $W^{\prime}$ is a strong Lebesgue-type decomposition for W'. The components of such a decomposition essentially satisfy HI3 (see Theorem III.3.3) and hence are almost over additive.

2) There exists a strong Lebesgue-type decomposition for W'. Its components satisfy Hl3.

We discuss briefly the proof of iif). 1) follows chiefly from Lemma III.3.2, ii), and Lemma I.7.5. 2) follows readily from theorem II.2.8, Lemma III.3.2, and 1). The argument is not difficult. It is rather similar to the one used in the proof of Theorem III.3.4.

iv) This development makes it possible to drop the assumptions HC2, H4*, and H7* from Theorem III.3.4.

III.4 On the AC component of the Special Decomposition Theorem

In this section we shall show that a maximal AC part of a weight function satisfying $\mathrm{HI} 3$ is actually a strongly maximal AC part.

III.4.2. Lemma. Assume HCI and HI5i. Let $w_{1}$ and $w_{2}$ be non-negative functions defined on 5 . Suppose that the induced point functions $\mathrm{g}_{\mathrm{w}_{1}}(\mathrm{~s}), \mathrm{s} \in \underline{\mathrm{S}}$, and $\mathrm{g}_{\mathrm{w}_{2}}(\mathrm{~s}), \mathrm{s} \in \underline{\mathrm{S}}$, are $\underline{B}-$ 
measurable. Define a non-negative function $w_{0}$ on $\sigma$ by $w_{0} S=$ $=\max \left(w_{1} s, w_{2} s\right\}, s \in 5$. Define non-negative functions $w_{i}$ on S'X

$$
w_{i}^{\prime}\left(s^{\prime}, D\right)=w_{*}\left(s^{\prime}, D, w_{i}\right), \quad\left(s^{\prime}, D\right) \in \underline{S}^{\prime} \times \underline{D},
$$

for $1=0,1,2$. Then $W_{i}$ is a weight function for $T, i=0,1,2$. If $T$ is $B V W_{1}$ and $B V W_{2}$ then $T$ is $B V W_{0}$. If $T$ is $\mathrm{ACW}_{1}$ and $\mathrm{ACW}_{2}$ then $\mathrm{T}$ is $\mathrm{ACW}_{\mathrm{O}}$.

Proof. For the induced point function $\mathrm{g}_{\mathrm{w}_{0}}(s), s \in \underline{\mathrm{S}}$, we have $g_{w_{0}}=\max \left(B_{w_{1}}, g_{w_{2}}\right)$. Hence $B_{w_{0}}(s), s \in \underline{S}$, is $\underline{B}$-measurable. By Theorem III.2.3 each $W_{i}$ is a weight function for $T$. Now suppose that $T$ is $B V W_{1}$ and $B V W_{2}$. Note that $w_{0} \leqq w_{1}+w_{2}$ so that, using Lemma I.7.9, $W_{0}^{\prime} \leqq W_{1}^{\prime}+w_{2}^{\prime}$. In particular $w_{0} \leqq w_{1} \underline{S}+w_{2} \underline{S}<$ $<\infty$.

Assume now that $T$ is $\mathrm{ACW}_{1}$ and $\mathrm{ACW}_{2}{ }^{\circ}$ Then surely $\mathrm{T}$ is $\mathrm{BVW}_{0}{ }^{\circ}$ Define $S_{i}^{+}=U S, S \in S$ and $w_{i} S>0$, for $i=0,1,2$. Evidently

$$
s_{0}^{+}=s_{1}^{+} U s_{2}^{+}
$$

To show that $T$ is $\mathrm{ACW}_{0}$ we shall use Theorem 7.24 of [2]. Thus let $M$ be a set in $M$ for which $M \cong S_{0}^{+}$and $\mu M=0$. Set $M_{i}=$ $=M \cap S_{i}^{+}, i=1,2$. Using Theorem 7.24 of [2] it follows that $T M_{1}$ and $T M_{2}$ are in $\underline{M}$ and $\mu{ }^{\prime} T M_{1}=\mu ' T M_{2}=0$. In view of (1), 
$T M=T M_{1} \cup T M_{2} \cdot$ Hence $T M$ is in $\underline{M}^{\prime}$ and $\mu^{\prime} T M=0$. It follows from Theorem 7.24 of [2] that $T$ is $\mathrm{ACW}_{0}$.

III.4.2. Theorem. Assume HI51, $\mathrm{HCl}$, and HC4. Assume that W' is a weight function for $T$ which satisfies Hl3. Suppose $T$ is BVW. Every maximal AC part of $W^{\prime}$ is a strongly maximal AC part of $W^{\prime}$.

Proof. Let $W_{A}^{\prime}$ be a maximal AC part of W'. Applying Theorem III.3.3 and then Corollary III.2.5 we find that there is a nonnegative function $w_{a}$ defined on $S$ such that the induced point function $g_{w_{a}}(s), s \in \underline{S}$, is $\underline{B}$-measurable and such that

$$
\begin{aligned}
& W_{*}\left(a^{\prime}, D, w_{Q}\right)=W_{A^{\prime}}\left(s^{\prime}, D\right) \text { a.e. } \mu^{\prime} \text { on } \underline{S}^{\prime} \text { uniformly with } \\
& \text { respect to } D_{i} W_{*}^{\prime}\left(s^{\prime}, D, w_{a}\right) \leq W_{A^{\prime}}\left(s^{\prime}, D\right),\left(s^{\prime}, D\right) \in \underline{S}^{\prime} \times \underline{D} \text {. } \\
& \text { Now let } W_{\#}^{\prime} \text { be an } A C \text { part of } W^{\prime} \text {. Repeating the above }
\end{aligned}
$$

reasoning, we obtain a non-negative function $w_{6}$ defined on 5 such that the induced-point function $\mathrm{B}_{w_{C}}(s), s \in \underline{S}$, is $\underline{B}-$ measurable and such that

$$
\begin{aligned}
& W_{*}^{\prime}\left(s^{\prime}, D, w_{c}\right)=W_{\#}^{\prime}\left(s^{\prime}, D\right) \text { a.e. } \mu^{\prime} \text { on } \underline{S}^{\prime} \text { uniformly with } \\
& \text { respect to } \underline{D} ; W_{*}^{\prime}\left(s^{\prime}, D, w_{c}\right) \leqq W_{\#}\left(s^{\prime}, D\right),\left(s^{\prime}, D\right) \in \underline{S}^{\prime} \times \underline{D} .
\end{aligned}
$$

Define a non-negative function $w_{0}$ on $\sigma$ by $w_{0} S=\max \left\{w_{a} s, w_{c} s\right\}$, $S \in{ }^{5}$. Define a non-negative function $W_{0}^{\prime}$ on $\underline{S}^{\prime} \times \underline{D}$ by $W_{0}^{\prime}\left(s^{\prime}, D\right)=$ $=W_{*}\left(s^{\prime}, D, W_{0}\right),\left(s^{\prime}, D\right) \in \underline{S} ' X \underline{D}$. By Lemma III.4.I and by Lemma 
I.7.5, W' is a weight function for $T$ and, moreover, T is ACW $0^{\circ}$ Now we define a non-negative function $\theta$ on 5 by

$$
\text { WS }=\inf \left[W^{\prime}(T S, D): S \subseteq D \in \underline{D}\right\}, \quad S \in S_{\text {. }}
$$

Using (1) and (2) we obtain

$$
W_{*}\left(s^{\prime}, D, w_{a}\right) \leqq W^{\prime}\left(s^{\prime}, D\right), \quad\left(s^{\prime}, D\right) \in \underline{S^{\prime} \times D},
$$

and

$$
W_{*}^{\prime}\left(s^{\prime}, D, W_{c}\right) \leqq W^{\prime}\left(s^{\prime}, D\right), \quad\left(s^{\prime}, D\right) \in \underline{S}^{\prime} \times D
$$

It follows from Lemma III.I.I that $w_{a} \leqq$ and $w_{c} \leqq \theta_{0}$ Hence $w_{0} \leqq$ and so $W_{0}^{\prime}$ is an AC part of $W^{\prime}$ (using Lemma III.I.2).

Moreover, surely

$$
W_{*}\left(s^{\prime}, D, w_{a}\right) \leqq W_{0}\left(s^{\prime}, D\right), \quad\left(s^{\prime}, D\right) \in \underline{S^{\prime} \times \underline{D},}
$$

and

$$
W_{*}\left(s^{\prime}, D, w_{c}\right) \leqq W_{0}^{\prime}\left(s^{\prime}, D\right), \quad\left(s^{\prime}, D\right) \in \underline{s}^{\prime} \times \underline{D} .
$$

From (1) and (3) it follows that $W_{A}^{\prime} \leqq W_{0}^{\prime}$ a.e. $\mu$ ' on $S^{\prime}$ uniformly with respect to $D$. Since $W_{0}^{\prime}$ is an $A C$ part of $W^{\prime}$ it follows that $W_{\dot{A}}=W_{0}^{\prime}$ a.e. $\mu^{\prime}$ on $\underline{S}^{\prime}$ uniformly with respect to $\underline{D}$. But then, by (2) and (4), $W_{\#} \leqq W_{A}^{\prime}$ a.e. $\mu^{\prime}$ on $\underline{S}^{\prime}$ uniformly with respect to D.

III.4.3. Corollary. Assume HI5i, HCl, and HC4. Assume that W' is a weight function for T which satisfies H13. Assume that $T$ is BVW. The AC component of any Lebesgue-type decomposition for $W '$ is a strongly maximal AC part of W'.

Proof. By Theorem II.1.15 the AC component of any Lebesgue- 
type decomposition for $W '$ must be a maximal AC part of W'. By Theorem III.4.2 any maximal AC part of $W$ ' must be a strongly maximal AC part.

III.4.4. Corollary. Assume HI5i, HCl, and HC4. Assume that $W$ is a weight function for $T$ satisfying H13. Assume that Tis BVW. There is essentially at most one Lebesgue-type decomposition for $W^{\prime}$ in the following sense: If $\left(W_{a}, W_{\#}\right)$ and $\left(W_{\dot{A}}, W_{S}^{\prime}\right)$ are Lebesgue-type decompositions for $W^{\prime}$ then $W_{a}^{\prime}=W_{A}$ a.e. $\mu^{\prime}$ on $\underline{S}^{\prime}$ uniformly with respect to $\underline{D}$ and $W_{\#}=W_{S}$ a.e. $\mu^{\prime}$ on $S^{\prime}$ uniformly with respect to $\underline{D}$.

Proof. Both $W_{a}^{\prime}$ and $W_{A}^{\prime}$ are strongly maximal AC parts of $W^{\prime}$. Hence $W_{a}^{\prime}=W_{A}^{\prime}$ a.e. $\mu^{\prime}$ on $\underline{S}^{\prime}$ uniformly with respect to $\underline{D}$. Since $T$ is BVW it follows that $W^{\prime}\left(s^{\prime}, D\right)<\infty$ a.e. $\mu^{\prime}$ on $\underline{S}^{\prime}$ uniformly with respect to $\underline{D}$. Thus since $W_{a}^{\prime}+W_{\#}=W^{\prime}=W_{A}^{\prime}+W_{S}^{\prime}$ it follows that $W_{\#}^{\prime}=W_{S}^{\prime}$ a.e. $\mu^{\prime}$ on $\underline{S}^{\prime}$ uniformly with respect to $\underline{D}$.

III.5 A property of weight functions of the special class

The material of this section is unrelated to the rest of the chapter. For the first two lemmas of this section it is not necessary to assume HII and HI2. Throughout this section $\left\{{ }^{*} D_{j}\right\} \geqq 1$ is a countable sequence of sets in $\underline{D}$ with the properties given in the last sentence of $\mathrm{H}^{4}$. 
III.5.1. Lemma. Assume H9. Suppose that $M$ is a set in $M$ for which $M \subseteq D_{j}$ for some $j$. Then there exists a double sequence $\left\{D_{k 1}\right\}_{k=1}^{\infty} \sum_{1=1}^{\infty}$ of sets in $\underline{D}$ having the properties

i) $M \subseteq U_{i \geqq I} D_{k i} \subseteq U_{i \geqq I} D_{k-1} i^{\prime}$

ii) $\Sigma_{i \geqq 1} W^{\prime}\left(8^{\prime}, D_{k i}\right) \leqq \Sigma_{i \geqq 1} W^{\prime}\left(B^{\prime}, D_{k-1} 1^{\prime}, 8^{\prime} \in \underline{S}^{\prime}\right.$,

for every $k>1$ and such that

iii) the sets $D_{k i}(I \geqq I)$ are pairwise disjoint, iv) $\mu\left(U_{i \geqq 1} D_{k i} \cap C M\right)<\frac{1}{k}$ for every $k \geqq 1$.

The proof is omitted. It is very similar to the proof of Lemma II.4.1. The main difference is that $H 4$ is used in the situation here which corresponds to the situation in which H4* is used in the proof of Lemma II.4.I.

III.5.2. Lemma. Assume H9. Assume that $T$ is ACW and let $f$ be a g.1.b.f. W. Suppose that $M$ is a set in $M$ for which $M \subseteq{ }^{*} D_{j}$ for some $j$. Let $\left(D_{k i}\right\}_{k=1}^{\infty} \sum_{i=1}^{\infty}$ be a double sequence of sets in D for which conditions $i)$ - iv) of Lemma III.5.1 hold. Then the sequence

$$
\left\{\Sigma_{i \geqq 1} W^{\prime}\left(B^{\prime}, D_{k i}\right)\right\}_{k=1}^{\infty}
$$

is convergent for $\mathrm{s}^{\prime}$ in $\underline{S}^{\prime}$. If a non-negative function $\mathrm{g}^{\prime}$ is defined on $\underline{S}^{\prime}$ by $\mathbf{g}^{\prime}\left(s^{\prime}\right)=\lim _{k} \Sigma_{i \geqq I} W^{\prime}\left(s^{\prime}, D_{k i}\right), s^{\prime} \in \underline{s}^{\prime}$, then $\mathrm{s}^{\prime}\left(\mathrm{s}^{\prime}\right), \mathrm{s}^{\prime} \in \underline{S}^{\prime}$, is $\underline{M}^{\prime}$-measurable and 


$$
\int_{S^{\prime}} B^{\prime}\left(s^{\prime}\right) d \mu=\int_{M} f(s) d \mu .
$$

The proof is exactiy like the proof of Lemma II.4.2.

III.5.3. Lemma. Assume H13. Assume that T is ACW and let $f$ be a $g \cdot I \cdot b \cdot f$. W. Let $M$ be a set in $M$ and suppose that $f(s)=0$ a.e. $\mu$ on $M$. Then there exists a subset $X^{\prime}$ of $\underline{S}^{\prime}$ such that $\mu^{\prime} X '=0$ and such that $W S=0$ whenever $S$ is any set in $\mathcal{E}_{M}$ for which $S \subseteq C T^{-1} X^{\prime}$.

Proof. Define $M_{j}=M \cap{ }_{*} D_{j}, j \geqq 1$. Then $M_{j}$ is in $M$ and $M_{j} \subseteq{ } D_{j} \cdot$ By Lemma III.5.1 there is for each $f$ a double sequence $\left(D_{j k i}\right\}_{k=1}^{\infty} i_{i=1}^{\infty}$ of sets in $\underline{D}$ such that

$$
\begin{aligned}
& \text { i) } M_{j} \subseteq U_{i \geqq I^{\prime}} D_{j k i} \subseteq U_{i \geqq 1} D_{j k-1}, \\
& \text { ii) } \Sigma_{i \geqq 1} W^{\prime}\left(s^{\prime}, D_{j k i}\right) \leqq \Sigma_{i \geqq l} W^{\prime}\left(s^{\prime}, D_{j k-1}\right)^{\prime}, s^{\prime} \in \underline{s}^{\prime},
\end{aligned}
$$

for every $k>1$ and

$$
\begin{aligned}
& \text { iii) the seta } D_{j k i}(i \geqq 1) \text { are pairwise disfoint, } \\
& \text { iv) } \mu\left(U_{i \geqq l} D_{j k i} \cap C_{j}\right)<\frac{I}{k}
\end{aligned}
$$

for every $k \geqq 1$. According to Lemma III.5.2 the sequence $\left\{\Sigma_{i \geqq I} W^{\prime}\left(s^{\prime}, D_{j k i}\right)\right\}_{k=1}^{\infty}$ is convergent for every $s^{\prime}$ in $\underline{S}^{\prime}$ and $j \geqq 1$. We define a non-negative function $B_{j}^{\prime}$ on $\underline{S}^{\prime}$ by $B_{j}^{\prime}\left(s^{\prime}\right)=$ $=\operatorname{Iim}_{k} \Sigma_{i \geqq 1} W^{\prime}\left(s^{\prime}, D_{j k i}\right), s^{\prime} \in \underline{s}^{\prime}, j \geqq 1$. By Lemma III.5.2, $\mathbf{g}_{j}^{\prime}\left(\mathbf{s}^{\prime}\right), \mathbf{s}^{\prime} \in \underline{S}^{\prime}$, is $\underline{M}^{\prime}$-measurable and 
(1)

$$
\int_{\underline{S}}, g_{j}^{\prime}\left(s^{\prime}\right) d \mu^{\prime}=\int_{M_{j}} f(s) d \mu, j \geqq I \text {. }
$$

By hypothesis $\int_{M_{f}} P(s) d \mu=0, j \geqq 1$, and so, by (1),

$$
B_{j}^{\prime}\left(s^{\prime}\right)=0 \quad \text { a.e. } \mu^{\prime} \text { on } \underline{S}^{\prime}, j \geqq 1 \text {. }
$$

Define $X_{j}^{\prime}=\left\{s^{\prime} \in \underline{S}^{\prime}: g_{j}^{\prime}\left(s^{\prime}\right)>0\right\}, j \geqq 1$. If we set $X^{\prime}=$ $=U_{j \geqq 1} x_{j}^{\prime}$ then, in view of (2), $X^{\prime}$ is in $\underline{M}^{\prime}$ and $\mu^{\prime} X^{\prime}=0$.

Now let $S_{0}$ be any set in $S_{M}$ for which $S_{0} \subseteq \mathrm{CT}^{-1} \mathrm{X}$ ' Now $S_{0}$ is in $S_{M_{j}}$ for some $j$. Since $T S_{0}$ is in C'X' we have

$$
\operatorname{Iim}_{k} \Sigma_{i \geqq 1} W^{\prime}\left(T_{0}, D_{j k i}\right)=0 \text {. }
$$

Let $k$ be any positive integer. Since $M_{j} \subseteq U_{i \geqq l} D_{j k i}$ and

$S_{0} \cap M_{j} \neq \varnothing$ there is an integer $i_{k}$ such that $S_{0} \cong D_{j k i_{k}}$ (7.2 of

[2]). For each $k$ we have $\Sigma_{i \geqq I} W \cdot\left(T S_{0}, D_{j k i}\right) \geqq W \cdot\left(T S_{0}, D_{j k i_{k}}\right) \geqq$ $\geqq w_{0}$. In view of (3), we must have $w_{0}=0$.

III.5.4. Lemma. Assume HI3. (Assume $W^{\prime}$ is generated by w.) Assume that $T$ is ACW and let $f$ be a g.l.b.f. W. Define $S^{+}=U S, S \in S$ and $W S>0$. Suppose that $M$ is a set in $M$ for which $M \cap S^{+}=\varnothing$. Then $f(s)=0$ a.e. $\mu$ on $M$.

$$
\text { Proof. Set } M_{j}=M \cap{ }_{*} D_{j}, j \geqq 1 \text {. Since } \underline{S}^{\prime}=U_{j \geqq 1} *_{j} D_{j} \text { it }
$$

suffices to prove that $f(s)=0$ a.e. $\mu$ on $M_{j}$ for every $j \geqq l$. Thus we fix $f$. Note that $M_{j}$ is in $\underline{M}$ and $M_{j} \subseteq{ }_{*} D_{j}$. By Lemma 
III.5.1 there is a double sequence $\left(D_{j k i}\right]_{k=1}^{\infty} \sum_{i=1}^{\infty}$ of sets in $D$ such that the properties listed as i) - iv) in the proof of Lemma III.5.3 are satisfied. According to Lemma III.5.2 the sequence $\left\{\Sigma_{i \geqq 1} W^{\prime}\left(s^{\prime}, D_{j k i}\right)\right\}_{k=1}^{\infty}$ is convergent for every $s^{\prime}$ in $\underline{s}^{\prime} \cdot$ We define a non-negative function $g_{j}^{\prime}$ on $\underline{S}^{\prime}$ by $g_{j}^{\prime}\left(s^{\prime}\right)=$ $=\operatorname{Iim}_{k} \Sigma_{i \geqq 1} W^{\prime}\left(s^{\prime}, D_{j k i}\right), s^{\prime} \in \underline{S}^{\prime}$. By Lemma III.5.2 $g_{j}^{\prime}\left(s^{\prime}\right)$, $s^{\prime} \in \underline{S}^{\prime}$, is $\underline{M}^{\prime}-$ measurable and

$$
\int_{\underline{S}^{\prime}} g_{j}^{\prime}\left(s^{\prime}\right) d \mu^{\prime}=\int_{M_{j}} f(s) d \mu .
$$

Define $S_{j}^{\prime}=\left\{s^{\prime} \in \underline{S}^{\prime}: g_{j}^{\prime}\left(s^{\prime}\right)>0\right\}$ and $S_{\infty}^{\prime}=\left\{s^{\prime} \in \underline{s}^{\prime}: W^{\prime}\left(s^{\prime}, \underline{s}\right)=\infty\right\}$. We now wish to prove

$$
S_{j} \subseteq S_{\infty}^{\prime} \cup T\left(S^{+} \cap \cap_{k \geq I} U_{i \geq I} D_{j k i}\right) \text {. }
$$

In order to prove (2) we let $s_{0}^{\prime}$ be a point in $S_{j}^{\prime}$ but not in $S_{\infty}^{\prime} \cdot$ Then, for $k \geqq 1$, we have

$$
\Sigma_{i \geqq 1} W^{\prime}\left(s_{0}^{\prime}, D_{j k i}\right)=\Sigma w S, \quad s \in \epsilon_{s} \text { and } S \subseteq U_{i \geqq I} D_{j k i} \cdot
$$

Since so is in C'S' we have

$$
\Sigma w S, \quad S \in \subseteq_{S_{j}} \text { and } S \subseteq U_{i \geqq I} D_{j k i},<\infty
$$

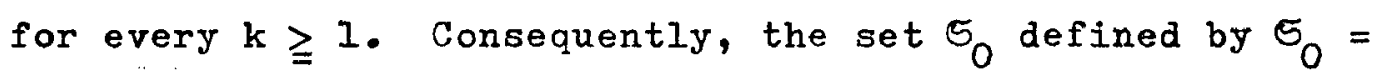
$=\left\{S \in E_{s_{0}^{\prime}}: w S>0\right.$ and $\left.S \subseteq U_{i \geqq 1} D_{j l i}\right\}$ is countable. It cannot be empty but it may be finite or infinite. Set $S_{0}=U S, S \in \mathcal{E}_{0}$. We now assert that 
(3)

$$
s_{0} \cap s^{+} \cap n_{k \geq I} U_{i \geq I^{D}}{ }_{j k i} \neq \varnothing \text {. }
$$

In order to prove (3) we must consider two cases.

Case I. Assume that $E_{0}$ is finite. We assume that (3) is false and derive a contradiction. List the elements of $S_{0}$ as $S_{1}, \ldots, s_{n}$. Since (3) is false it follows that, for $u=1, \ldots$, $n$, there exists an integer $k_{u}$ such that $S_{u} \notin U_{i \geq 1} D_{j k_{u} i}$ and hence $s_{u} \cap U_{i \geqq 1} D_{j k_{u} i}=\varnothing$. Set $k_{0}=\max \left(k_{1}, \ldots, k_{n}\right\}$. In view of $\left.i\right)$ $S_{u} \cap U_{i \gg I} D_{j k_{0}}=\varnothing, u=1, \ldots, n$. Hence (4)

$$
S_{u} \cap D_{j k_{0} i}=\varnothing, \quad i \geqq 1, u=1, \ldots, n \text {. }
$$

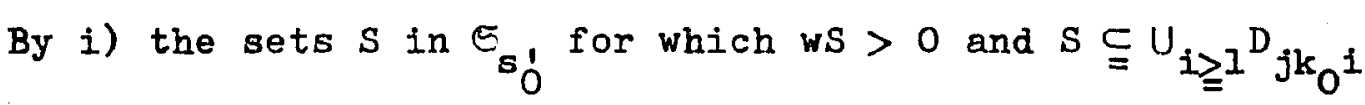
must occur among $S_{1}, \ldots, S_{n}$. In view of (4) it follows that $W \cdot\left(s_{0}^{\prime}, D_{j k_{0} i}\right)=0, i \geqq I ;$ hence

$$
\Sigma_{i \geqq 1} W^{\prime}\left(s_{0}^{\prime}, D_{j k_{0}}\right)=0
$$

and so $g_{j}^{\prime}\left(s_{0}^{\prime}\right)=0$. But $s_{0}^{\prime}$ is in $s_{j}^{\prime}$. Hence we have a contradiction. Thus (3) holds in this case.

Case II. $S_{0}$ is denumerable. Again we assume (3) is false and derive a contradiction. List the elements of $E_{0}$ as $S_{1}$, $S_{2}, \ldots$ Set $p=g_{j}^{\prime}\left(s_{0}^{\prime}\right)>0$. Note that $\Sigma_{n \geqq I} w S_{n}=$ $=\Sigma_{i \geq I} W^{\prime}\left(s_{0}^{\prime}, D_{j I i}\right)<\infty$. There exists an integer $N$ such that (5)

$$
\Sigma_{n \geqq \mathbb{N}} w S_{n}<p .
$$


We are assuming $(3)$ is false and so $U_{n \geqq 1} S_{n} \cap s^{+} n n_{k \geqq l} U_{i \geqq 1} D_{j k i}=$ $=\varnothing$. Note that $U_{n \geqq I} S_{n} \subseteq S^{+}$. For each $n$ we then have $S_{n} \cap$ $n n_{k \gg I} U_{i \geqq I}{ }^{j k i}=\varnothing$. Hence for each $n$ there exists a positive integer $k_{n}$ such that $s_{n} \cap U_{i \geqq I} D_{j k_{n} i}=\varnothing$. Set $k_{0}=\max \left\{k_{1}, \ldots\right.$, $\left.k_{N}\right\}$. In view of $\left.i\right)$ the only sets $S$ in $s_{s}$ for which $w S>0$ and $S \subseteq U_{i \geq 1} D_{j k_{0}}$ occur among $S_{N+1}, S_{N+2}, \ldots$ Using (5) we obtain $\Sigma_{i \geqq I} w^{\prime}\left(s_{j}^{\prime}, D_{j k_{0} i}\right) \leqq \Sigma_{n>N} w_{n}<p$.

Thus, by ii), $g_{j}^{\prime}\left(s_{0}^{\prime}\right) \leqq \Sigma_{i \geqq I} W^{\prime}\left(s_{0}^{\prime}, D_{j k_{0} i}\right)<p$. But this is a contradiction. Hence (3) is valid in this case also. With (3) established, (2) follows at once. We now examine the sets $S_{\infty}^{\prime}$ and $T\left(S^{+} \cap \cap_{k \geqq l} U_{i \geqq l} D_{j k i}\right)$. Note first that $S_{\infty}^{\prime}$ is in $M^{\prime}$ and, since $T$ is $B V W$, we have

$$
u \cdot S_{\infty}^{\prime}=0 \text {. }
$$

Observe that $S^{+} \subseteq \mathrm{CM}_{j}$ and so $s^{+} \cap \cap_{k \geq I} U_{i \geqq 1} D_{j k i} \subseteq \cap_{k \geqq 1} U_{i \geqq 1} D_{j k i} \cap$

$\cap \mathrm{CM}_{j} \cdot$ In view of $\left.i v\right)$ and $\left.i\right)$ we have $\mu\left(\cap_{k \geqq I} U_{i \geqq I} D_{j k i} \cap \mathrm{CM}_{j}\right)=0$. Hence $S^{+} \cap \cap_{k \gg l} U_{i \geqq 1} D_{j k i}$ is in $\underline{M}$ and $\mu\left(s^{+} \cap \cap_{k \geqq I} U_{i \geqq I} D_{j k i}\right)=0$. By Theorem 7.24 of [2] it follows that $T\left(s^{+} \cap n_{k \geqq 1} U_{i \geqslant I} D_{j k i}\right)$ is in M $^{\prime}$ and 


$$
\mu \cdot T\left(S^{+} \cap \cap_{k \geqq I} U_{i \geqq I} D_{j k i}\right)=0 .
$$

It now follows from (2), (6), and (7) that

$$
\mu \cdot S_{j}^{\prime}=0 \text {. }
$$

It follows from (8) and (I) that $f(s)=0$ a.e. $\mu$ on $M_{j}$

III.5.5. Theorem. Assume H9 and suppose in fact that $W^{\prime}$ is generated by a non-negative function $w$ defined on 5 . Set $S^{+}=U S, S \in S$ and $w S>0$. Assume that $S^{+}$is in $\underline{M}_{0}$ (In view of Corollary III.2.5 this last assumption is not so restrictive.) Assume also that $T$ is ACW and let $f$ be a g.l.b.f. W. Then $f(s)=$ $=0$ a.e. $\mu$ on $\mathrm{CS}^{+}$. There exists a set $X^{\prime}$ in $\underline{M}^{\prime}$ such that $\mu^{\prime} X^{\prime}=0$ and such that $f(s)>0$ whenever $s$ is in $S^{+} \cap \mathrm{CT}^{-1} \mathrm{X}^{\prime}$. Proof. The set $S^{+}$is in $M$ and bence $\mathrm{CS}^{+}$is in $\underline{M}$. By Lemma III.5.4, $f(s)=0$ a.e. $\mu$ on $\mathrm{CS}^{+}$. Now define $\mathrm{M}_{0}=$ $=\left\{s \in \underline{S}: s \in S^{+}\right.$and $\left.f(s)=0\right\}$. Since $S^{+}$is in $\underline{M}$ and $f(s)$, $s \in \underline{S}$, is $\underline{M}$-measurable it follows that $M_{0}$ is in $M_{\text {. Surely }}$ $f(s)=0$ on $M_{O}$ and so Lemma III. 5.3 applies. There exists a set $X^{\prime}$ in $M^{\prime}$ such that $\mu^{\prime} X^{\prime}=0$ and such that $w S=0$ whenever $S$ is in $5_{M_{0}}$ and $S \subseteq \mathrm{CT}^{-1} X^{\prime}$. It is easily verified that $M_{0} \cong T^{-1} X^{\prime}$. It follows that $f(s)>0$ whenever $s$ is in $\mathrm{S}^{+} \cap \mathrm{CF}^{-1} \times 1$. 


\section{SUMMARY}

We shall now repeat the most important results of this paper. These principal theorems will be reviewed in a rather informal manner. Precise statements of them have of course been given in the text. Comments regarding possible extensions of them and concerning related problems are interspersed with the statements of these principal results.

Theorems II.1.13 and II.1.14 comprise the main result of Section II.I. Actually, these theorems present the same result in two different forms. They are proved under the same assumptions; for each of them we assume $\mathrm{Hl}-\mathrm{H} 9, \mathrm{HC} 4$, and that $W_{A}$ is a finite valued $A C$ part of $W^{\prime}$. We prove in Theorem II.1.13 that there is a weight function $W_{\#}$ for $T$ such that $W^{\prime}=W_{A}^{\prime}+W_{\#} a_{\cdot} \cdot \mu^{\prime}$ on $\underline{S}^{\prime}$ uniformly with respect to $\underline{D}$. In Theorem II.1.14 the role of $W_{A}$ is played down. somewhat in order to produce a modified conclusion; indeed we obtain two new weight functions $W_{a}^{\prime}$ and $W_{\#}$ for $T$ with the properties that $W^{\prime}=w_{a}^{\prime}+W_{\#}$ and that $W_{a}^{\prime}$ is an AC part of $W^{\prime}$ which agrees with $W_{A}^{\prime}$ a.e. $\mu^{\prime}$ on $\underline{S}^{\prime}$ uniformly with respect to D. Both of these results are presented because in a given situation the one may be more convenient than the other. Each is a "decomposition" result in its own right; together, they comprise an indispensable preliminary for the main decomposition theorems. 
The remarks in II.I.10 and in II.63 suggest that it may be possible to remove HC4 from Theorems II.1.13 and II.1.14-and hence from the theory-by revising H9. Such a revision might prove fruitful. It is a problem that is likely to bo attacked rather soon.

Next, we discuss the General Decomposition Theorem. The theorem can be stated as follows: If $\mathrm{HL}-\mathrm{H} 9$ and HC4 are assumed and if $T$ is assumed to be BVW, then $W$ can be expressed as the sum of two weight functions, one of which is an $A C$ part of $W '$ and the other of which is singular. This decomposition requires of $W$ only that $T$ be BVW. Hence the requirements concerning $W$ are all but minimal. Unfortunately, the General Decomposition Theorem is weak because singular weight functions have never been described very well. The goal of the discussion which leads to the Strong Decomposition Theorem is precisely the discovery of properties possessed by singular weight functions. At the outset the specific aim was the discovery of some sort of relationship between singular weight functions (for $T$ ) and the transformation $T$ itself. As a preliminary, the writer attempted to discover such a relationship for those singular weight functions which happen to satisfy H13. Theorem 7.24 of [2] proved to be an invaluable aid in this restricted situation. It was then natural to seek a counterpart of Theorem 7.24 which does not require H13. The examples in II.7 suggest in several ways that such a counterpart may be difficult to obtain. It remains a worthwhile (but unrealized) goal. For the purposes of this paper howerer, it is sufficient to work with a 
rather modest counterpart - Theorem II.3.2 - of 7.24 . Theorem II.3.2 asserts roughly that if we assume $\mathrm{HI}-\mathrm{H} 9, \mathrm{H} 7^{*}$, and $\mathrm{HCl}$, then, if $W$ is over additive, $T$ is $B V W$, and if $T$ transforms certain sets of measure zero into sets of measure zero, it is true that $T$ is ACW. An example in II.7 reveals that $T$ can be ACW and yet not transform these certain sets of measure zero into sets of measure zero. In other words, the most natural converse of Theorem II.3.2 fails to hold. A better theorem of this type would be something like the following: Assume that $T$ is $B V W$ and that $W$ is over additive; then $\mathrm{T}$ is $\mathrm{ACW}$ if and only if it transforms certain sets of measure zero into sets of measure zero. In view of Example II.7.5, it would seem to be very difficult to remove the overriding assumption that $W$ be over additive from such a theorem. Our strongest theorems concerning Lebesgue-type decompositions are Theorem II.5.2 and Corollary II.5.3. The following paragraph consists of a rather informal statement of these two closely related theorems.

Assume $\mathrm{HI}-\mathrm{H} 9$, $\mathrm{H} 4^{*}, \mathrm{H} 7^{*}, \mathrm{HCl}$, and $\mathrm{HC2}$. Assume that T Is BVW and that $W^{\prime}$ is almost over additive. Then every Lebesgue-type decomposition for $W$ ' is a strong Lebesgue-type decomposition for W'; the singular component of such a decomposition must be almost over additive. Assume now that $\mathrm{HC} 4$ also holds. Then W' does admit a strong Lebesgue-type decomposition; in other words, W' can be written as the sum of two weight functions for $T$, one of which is an AC part of $W^{\prime}$ and the other of which is not only singular but also vanishes almost everywhere outside of the image of a subset of $\underline{S}$ having measure zero. 
Thus we obtain a strong Lebesgue-type decomposition only for weight functions $W$ ' which are almost over additive and for which Is BVW. The weakening of the former requirement is a desirable goal. The example in II.7.5 shows that it cannot be dropped entirely. Indeed it would be worthwhile to establish conditions on $W^{\prime}$ which are necessary and sufficient for it to have a strong Lebesgue-type decomposition. Such a necessary and auficient condition might prove valuable in other circumstances. Imposition of such a condition on weight functions in general might eliminate such "pathological" cases as the one described in II.7.5.

Chapter III contains theorems about the important special class consisting of those weight functions which satisfy HI3. We discuss some measurability questions in the first part of Chapter III. The results are these. We first prove that if we assume $\mathrm{HI}-\mathrm{H} 8, \mathrm{HL} 15 \mathrm{i}, \mathrm{HCl}$, and that $\mathrm{w}$ is a non-negative function defined on 5 whose induced point function $B_{W}$ is B-measurable, then the summatory function generated by $w$ is a weight function for T. This theorem can be applied to obtain a second raluable theorem. It is the following: If we assume $\mathrm{Hl}-\mathrm{H} 9, \mathrm{HI} 1$, and $\mathrm{HCl}$ and if we define a non-negative function on 5 by $A S=\inf [W \cdot(T S, D): S \subseteq D \in D\}, S \in G$

then there exists a non-negative function w defined on 5 whose induced point function is B-measurable and which agrees with on the complement of the inverse of a set of $\mu^{\prime}$ measure zero; moreover, the summatory function generated by is a weight function 
for T. There is a useful corollary to these two theorems. Its most prominent application is based upon the following vaguely stated feature: The class of weight functions satisfying $\mathrm{H} 13$ and having B-measurable induced point functions is "dense" in the class of all weight functions satisfying HI3.

The next main result is the Special Decomposition Theorem. It can be obtained in two ways. The better result is this: If $\mathrm{Hl}-\mathrm{H} 8, \mathrm{HI} 3, \mathrm{HCl}$, and $\mathrm{HC} 4$ are assumed and if $\mathrm{T}$ is $\mathrm{BVW}$, then every Lebesgue-type decomposition for $W^{\prime}$ is a strong Lebesgue-type decomposition for W'; the components of such a decomposition "essentially satisfy" H13. Moreover, there does exist a strong Lebesgue-type decomposition for W', the components of which satisfy Hl3.

We then establish several additional facts about the decomposition given by the Special Decomposition Theorem. Indeed it is shown that if $\mathrm{HI}-\mathrm{H} 8, \mathrm{HI} 3, \mathrm{HCl}$, and $\mathrm{HC} 4$ are assumed, then the AC component of a Lebesgue-type decomposition for $W '$ must be a strongly maximal $A C$ part of $W^{\prime}$; it follows readily that there is in essence only one Lebesgue-type decomposition for W' in this case.

The results reviewed in the preceding paragraph strengthen substantially the conclusions of the Special Decomposition Theorem. We ask immediately whether we can establish similar results in conjunction with the General and Strong Decomposition Theorems. These problems are unsolved. Solution of them would surely give the General and Strong Decomposition Theorems a more definite character. 
Finally, we repeat the principal conclusion of III.5. Roughly speaking, it is shown that if $\mathrm{HL}-\mathrm{H} 8$ and $\mathrm{HI} 3$ are satisfied, if $T$ is $A C W$, and if $f$ is a $B .1 . b . f . W$, then the functions $B_{W}$ and $f$ are either both zero or both positive at all points except those in the union of some set of $\mu$ measure zero and some set whose image under $T$ has $\mu^{\prime}$ measure zero. This theorem occupies a rather solitary position at present. No applications of it have been made. 


\section{BIBLIOGRAPHY}

1. T. Rado and P. V. Reichelderfer, Continuous Transformations in Analysis, Springer-Verlag, Berin, Gottingen, Heidelberg, 1955.

2. P. V. Reichelderfer, "A transformation theory for measure space," Rendiconti del Circolo Matematico di Palermo, 10 (1961), pp. 283-313.

3. S. Saks, Theory of the Integral, 2d ed. revised, Monografie Matematyczne, vol. 7, Warszawa-Lwow, 1937. 